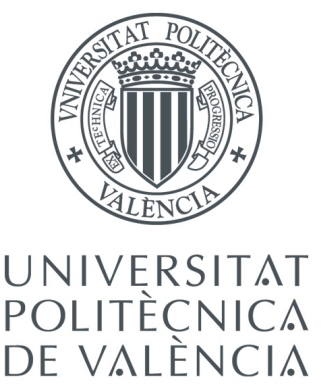

\title{
Methods for the treatment of uncertainty in dynamical systems:
}

\section{Application to diabetes}

PhD dissertation by:

Diego de Pereda Sebastián

Supervisors:

Dr. Jorge Bondia Company

Dra. Beatriz Ricarte Benedito

Dr. Sergio Romero Vivó 

This work was partially supported by:

Ministerio de Ciencia e Innovación through Grant DPI-2010-20764-C02-01,

Grant DPI-2013-46982-C2-1-R and Grant MTM-2013-43678-P.

Universitat Politècnica de València through Grant PAID-05-09-4334.

Generalitat Valenciana through Grant GV/2012/085.

European Union through FEDER funds. 

Dedicado a todos los que me he ido encontrando por el Camino 



\section{Agradecimientos}

Los problemas de los puzzles son parecidos a los problemas de la vida. El problema del cubo de Rubik sólo depende de ti, pero para poder encontrar la felicidad en la vida no eres independiente. Esa es la única gran diferencia.

Ernö Rubik

Se cumple casi un lustro desde que, de la noche a la mañana, preparé las maletas y vine a Valencia. 'Pensat ifet', que dicen por aquí. Los cambios nunca son sencillos, pero la ciudad y la gente ayudaron a que no tardara en hacer de esta tierra un hogar.

Esta tesis no sería posible si no fuera por el tiempo y esfuerzo que mis directores me han dedicado. Sergio confió en mí desde un primer momento y, desde entonces, siempre ha estado cuando le he necesitado. Además, le da un toque ameno a las reuniones gracias a su incomprendido sentido del humor. Jorge, cual capitán de barco, ha ido dirigiendo mi evolución como doctorando, así como la de este trabajo. Mientras que Bea ha destacado por su eficiencia y por ser la primera que respondía mis dudas. Eso sí, también ha sido la que más ha sufrido que yo sea un poquitín 'cabut'. Ha sido un placer trabajar con vosotros.

Estos años no hubieran sido lo mismo sin los magníficos compañeros que me han ido marcando el camino y que han sido un referente para mí. Quisiera agradecerle a Alejandro V., por seguirme en mis locuras y preocuparse de mí ( LLo que pasa en la carpa queda en la carpa»); a Fàtima, per les partides de Catan i els dinars (al migdia); a Gilberto, por enseñarme el valor de la disciplina y por discutir modelos matemáticos apuntados en servilletas de bar; a Jopipe, por su carácter provocador y fiestero («iCastellón capital, Valencia sucursal!»); a Alejandro L., por las paellas y torradas; a Ana, por su boda 'pasada por agua'; y a Kiko, por su capacidad analítica llevada hasta el extremo.

Nuevos compañeros me han acompañado en el 'nuevo orden mundial' que recientemente se ha instaurado. Quisiera agradecer a Vanessa, porque su optimismo 'Happy Flower' llena de luz a la sala; a Rubén, por atenuar la luz con su realismo; a Yadira, por poder confiar siempre en ella; a Jesús, por no 
poder decir que no a ningún plan; a Gabi, por cuidar de la sala cuando yo no estoy; a Temo, por querer siempre más (aunque ya sea demasiado); a Manuel, por su optimismo en cualquier situación; y a Alberto, por haberse convertido en un miembro VIP. A todos vosotros os dejo un consejo que os será de gran utilidad en el futuro: «iEmpezad a escribir la tesis ya, insensatos!»

También quiero agradecerle esta tesis a Sonia, por apoyarme día tras día y haber sido mi guía durante tres años.

I want to thank to Jean-Luc and Francis, for working with me during my stay at Inria. Thank you to Stefano, Alfonso, Marco, Elsa, Bapan, Francesco, Dmitry, Olga and Sonya for becoming a part of my life in just a few months.

Quiero recordar mis primeros pasos en la investigación, en la Facultad de Medicina de la Complutense. Allí, en el grupo de los Frikimáticos, me di cuenta de cuánto me apasionaba la investigación. Muchas gracias a todos los compañeros que hacían de la hora de la comida algo mágico, especialmente a Carmen y a Juan.

También quiero agradecer a mis compañeros de Matemáticas por hacer que saque mi lado más auténtico (y friki) cada vez que les veo. Especialmente a Susana, que me ha acompañado por alfombras y demás situaciones la mar de variopintas desde el primer día de la carrera.

Uno de los aspectos más duros de realizar un cambio es darse cuenta de lo que uno deja atrás. Me duele no poder vivir el día a día con los Plavi y no estar tan al tanto de vuestros quehaceres como me gustaría. Ahora sólo puedo estar con vosotros en pequeñas píldoras, pero trato de aprovecharlas al máximo. Muchas gracias por poder seguir confiando en vosotros y sentir, por unos instantes, que todo sigue como antes.

A pesar de haber vivido sólo el parto y no todo el embarazo, tengo que agradecerle a Lucía haberme dado luz cuando estaba oscuro. Gracias por vivir cada momento con afán.

También quiero dedicar la tesis a los amigos del colegio, especialmente a Adolfo, que por muy lejos que esté siempre le siento cercano.

Por último, pero no por ello menos importante, quisiera agradecerle a mi familia haberme ayudado a seguir mis sueños, aunque ello suponga tenerme alejado del hogar. Especialmente, quiero dedicar esta tesis a mis abuelos, Pablo y Flora, por tener siempre un plato listo para cuando les hago una visita.

Muchas gracias a todos.

Sin vosotros no hubiera sido posible.

O, por lo menos, no hubiese sido tan divertido. 


\section{Abstract}

Patients suffering from Type 1 Diabetes are not able to secrete insulin, thus, they have to get it administered externally. Current research is focused on developing an artificial pancreas, a control system that automatically administers insulin according to patient's needs. The work presented here aims to improve the efficiency and safety of control algorithms for artificial pancreas.

Glucose-insulin models try to mimic the administration of external insulin, the absorption of carbohydrates, and the influence of both of them in blood glucose concentration. However, these processes are infinitely complex and they are characterized by their high variability. The mathematical models used are often a simplified version which does not include all the process variability and, therefore, they do not always match reality. This deficiency on the models can be addressed by considering uncertainty on their parameters and initial conditions. In this way, the exact values are unknown but they can be bounded by intervals that comprehend all the variability of the considered process. When the value of the parameters and initial conditions is known, there is usually just one possible behaviour. However, if they are bounded by intervals, a set of possible solutions exists. In this case, it is interesting to compute a solution envelope that guarantees the inclusion of all the possible behaviours. A common technique to compute this envelope is the monotonicity analysis of the system. Nevertheless, some overestimation is produced if the system is not fully monotone. In this thesis, several methods and approaches have been developed to reduce, or even eliminate, the overestimation in the computation of solution envelopes, while satisfying the inclusion guarantee.

Another problem found during the use of an artificial pancreas is that only the subcutaneous glucose concentration can be measured in real time, with some noise in the measurements. The rest of the system states are unknown, but they could be estimated from this set of noisy measurements by state observers, like Kalman filters. A detailed example is shown at the end of this thesis, where an Extended Kalman Filter is used to estimate in real time insulin concentration based on the food ingested and in periodical measurements of subcutaneous glucose. 



\section{Resumen}

Los pacientes que sufren de diabetes tipo 1 no son capaces de secretar insulina, por lo que tienen que administrársela externamente. La investigación actual se centra en el desarrollo de un páncreas artificial, un sistema de control que administre automáticamente la insulina en función de las necesidades del paciente. El trabajo que aquí se presenta tiene como objetivo mejorar la eficiencia y la seguridad de los algoritmos de control para el páncreas artificial.

Los modelos de glucosa-insulina tratan de emular la administración externa de la insulina, la absorción de carbohidratos y la influencia de ambos en la concentración de glucosa en sangre. El problema es que estos procesos son infinitamente complejos y se caracterizan por su alta variabilidad. Los modelos matemáticos utilizados suelen ser una versión simplificada que no incluye toda la variabilidad del proceso y, por lo tanto, no coinciden con la realidad. Esta deficiencia de los modelos puede subsanarse considerando inciertos sus parámetros y las condiciones iniciales, de manera que se desconoce su valor exacto pero sí podemos englobarlos en ciertos intervalos que comprendan toda la variabilidad del proceso considerado. Cuando los valores de los parámetros y de las condiciones iniciales son conocidos, existe, por lo general, un único comportamiento posible. Sin embargo, si están delimitados por intervalos se obtiene un conjunto de posibles soluciones. En este caso, interesa obtener una envoltura de las soluciones que garantice la inclusión de todos los comportamientos posibles. Una técnica habitual que facilita el cómputo de esta envoltura es el análisis de la monotonicidad del sistema. Sin embargo, si el sistema no es totalmente monótono la envoltura obtenida estará sobrestimada. En esta tesis se han desarrollado varios métodos para reducir, o incluso eliminar, la sobrestimación en el cálculo de envolturas, al tiempo que se satisface la garantía de inclusión.

Otro inconveniente con el que nos encontramos durante el uso de un páncreas artificial es que solo es posible medir en tiempo real, con cierto ruido en la medida, la glucosa subcutánea. El resto de los estados del sistema son desconocidos, pero podrían ser estimados a partir de este conjunto limitado de mediciones con ruido utilizando observadores de estado, como el Filtro de Kalman. Un ejemplo detallado se muestra al final de la tesis, donde se estima en tiempo real la concentración de insulina en plasma en función de la comida 
ingerida y de mediciones periódicas de la glucosa subcutánea con ayuda de un Filtro de Kalman Extendido. 


\section{Resum}

Els pacients que pateixen de diabetis tipus 1 no són capaços de secretar insulina, motiu pel qual han d'administrar-se-la externament. La investigació actual es centra en el desenvolupament d'un pàncrees artificial, un sistema de control que administre automàticament la insulina en funció de les necessitats del pacient. El treball que ací es presenta té com a objectiu millorar l'eficiència $i$ la seguretat dels algorismes de control per al pàncrees artificial.

Els models de glucosa-insulina tracten d'emular l'administració externa de la insulina, l'absorció de carbohidrats i la influència d'ambdós factors en la concentració de glucosa en sang. El problema és que estos processos són infinitament complexos i es caracteritzen per la seua alta variabilitat. Els models matemàtics emprats solen ser una versió simplificada que no inclou tota la variabilitat del procés i, per tant, no coincideixen amb la realitat. Esta deficiència dels models pot esmenar-se considerant incerts els seus paràmetres $i$ les condicions inicials, de manera que es desconeix el seu valor exacte però sí podem englobar-los en certs intervals que comprenguen tota la variabilitat del procés considerat. Quan els valors dels paràmetres i de les condicions inicials són coneguts, existeix, en general, un únic comportament possible. No obstant, si estan delimitats per intervals s'obté un conjunt de possibles solucions. En este cas, interessa obtindre un embolcall de les solucions que assegure la inclusió de tots els comportaments possibles. Una tècnica habitual que facilita el còmput d'este embolcall és l'anàlisi de la monotonicitat del sistema. No obstant, si el sistema no és totalment monòton l'embolcall obtingut estarà sobreestimat. En esta tesi s'han desenvolupat diversos mètodes per a reduir, $\mathrm{o}$ fins i tot eliminar, la sobreestimació en el càlcul dels embolcalls, al temps que se satisfà la garantia d'inclusió.

Altre inconvenient amb què ens trobem durant l'ús d'un pàncrees artificial és que només és possible mesurar en temps real, amb cert soroll en la mesura, la glucosa subcutània. La resta dels estats del sistema són desconeguts, però podrien ser estimats a partir d'este conjunt limitat de mesures amb soroll utilitzant observadors d'estat, com el Filtre de Kalman. Un exemple detallat es mostra al final de la tesi, on s'estima en temps real la concentració d'insulina en plasma en funció del menjar ingerit i de les mesures periòdiques de la glucosa subcutània amb ajuda d'un Filtre de Kalman Estés. 



\section{Contents}

Abstract . . . . . . . . . . . . . . . . ix

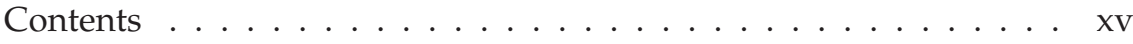

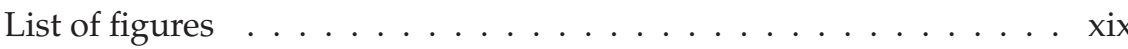

List of tables . . . . . . . . . . . . . . . . . . xxi

1 Motivation and objectives 1

1.1 Motivation ...................... 1

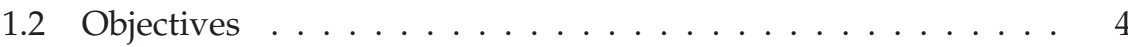

1.3 Outline ...................... 5

2 Simulation of interval dynamic systems 7

2.1 Modelling reality with Mathematics . . . . . . . . . . . . 7

2.2 Interval dynamic systems $\ldots \ldots \ldots \ldots \ldots$

2.2.1 Monte Carlo simulation . . . . . . . . . . . . . . . . 12

2.2.2 Guaranteed solution envelopes . . . . . . . . . . . . 13

2.2.3 Modal interval analysis . . . . . . . . . . . . . . . . 14

2.2.4 Region-based approaches . . . . . . . . . . . . . . 17

2.2.5 Trajectory-based approaches . . . . . . . . . . . 19

2.2.6 Partition of the input space . . . . . . . . . . . 23

3 Solution envelopes for parallel inputs models 25

3.1 Introduction . . . . . . . . . . . . . . . . . . . 25

3.2 Parallel inputs model . . . . . . . . . . . . . . . . . . . . . . . . . .

3.3 Proposed method . . . . . . . . . . . . . . . . . 28

3.4 Examples . . . . . . . . . . . . . . . . . . . . . . . 29

3.4.1 Double-peak phenomenon . . . . . . . . . . . 29

3.4 .2 Subcutaneous insulin absorption . . . . . . . . . . . 30

3.5 Discussion and conclusion . . . . . . . . . . . . . . . . 32

4 Solution envelopes for in-series model 35

4.1 Introduction . . . . . . . . . . . . . . . 35

4.2 In-series models . . . . . . . . . . . . . . . . . . 36

4.3 Proposed method . . . . . . . . . . . . . . . . 37

4.4 Examples . . . . . . . . . . . . . . . . . . . . . 41

4.4 .1 Linear glucose model . . . . . . . . . . . . 41 
4.4.2 Non-linear epidemiological SIS model . . . . . . . . . 43

4.5 Discussion and conclusion .................. 47

5 Solution envelopes based on cone monotonicity 49

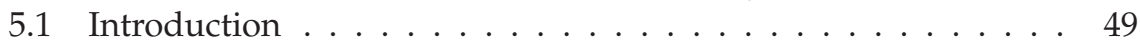

5.2 Cone monotonicity analysis . . . . . . . . . . . . . . . . . . 49

5.3 Proposed method . . . . . . . . . . . . . . 51

5.4 Example: non-linear chemostat model . . . . . . . . . . . . 55

5.5 Discussion and conclusion .................. 57

6 Prediction of glucose concentration under variability 59

6.1 Introduction . . . . . . . . . . . . . . . . 59

6.2 Glucose-insulin model . . . . . . . . . . . . . . . 60

6.2.1 The carbohydrate digestion and absorption system . . . 60

6.2.2 The subcutaneous insulin absorption system . . . . . . . 61

6.2 .3 The insulin action system . . . . . . . . . . . . 61

6.2.4 The glucose metabolism system . . . . . . . . . . . . 61 61

6.3 Solution envelopes under parametric uncertainty . . . . . . . . 62

6.3.1 The carbohydrate digestion and absorption system . . . 62

6.3.2 The subcutaneous insulin absorption system . . . . . . . 63

6.3.3 The insulin action system . . . . . . . . . . . . . 64

6.3.4 The glucose metabolism system ... . . . . . . . . 64

6.4 Results ............................ 67

6.4.1 Long-term glucose prediction . . . . . . . . . . . . . . 67

6.4.2 Short-term glucose prediction . . . . . . . . . . . . . 69

6.5 Discussion and conclusion ............... 70

7 Real-time estimation of plasma insulin concentration 73

7.1 Introduction . . . . . . . . . . . . . . . . . . . . . . . 73

7.2 Extended Kalman filter . . . . . . . . . . . . . . . . . . . . . . . . . . . . . .

7.3 Real-time estimation of plasma insulin . . . . . . . . . . . 75

7.3.1 Estimation from an insulin model . . . . . . . . . . 76

7.3.2 State observation of a glucose-insulin model . . . . . . . 76

7.3.3 State observation of an extended model . . . . . . . . . 77

7.4 Validation of insulin estimations . . . . . . . . . . . 78

7.4 .1 In-silico validation . . . . . . . . . . . . . . . 78

7.4 .2 Validation with real data . . . . . . . . . . . . . . . . . . . . . . . .

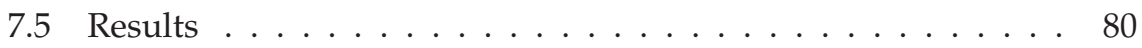

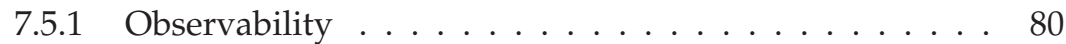

7.5 .2 In-silico results . . . . . . . . . . . . . . . . . 80

7.5.3 Results with real data . . . . . . . . . . . . . . . 82

7.6 Discussion and conclusion ................ 83

Conclusions of this thesis 87 
References

Author 


\section{List of Figures}

2.1 Diagram of the glucose-insulin system . . . . . . . . . . 8 8

2.2 Diagram of the phenomenological insulin absorption model developed by Puckett and Lightfoot . . . . . . . . . . . . . . 9 9

2.3 Diagram of the behavioural insulin absorption model developed by Kraegen and Chisholm . . . . . . . . . . . . . . . . . 10

2.4 Diagram of the two-channel insulin absorption model developed by Wilinska et al. . . . . . . . . . . . . . . . . . . . . . 10

2.5 Set of possible solutions under interval uncertainty . . . . . . 11

2.6 Probability density function of the continuous uniform distri-

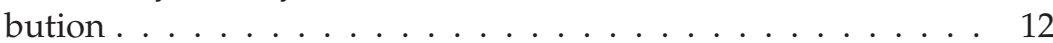

2.7 Diagram of proper and improper intervals . . . . . . . . 14

2.8 Diagram of inclusion and 'less than' relations . . . . . . . . 15

2.9 Diagram of lattice operators: Meet, Join, Max and Min . . . . . . 16

2.10 Representation of a priori and tight bounds . . . . . . . . 17

2.11 Representation of the wrapping effect . . . . . . . . . . 18

2.12 QR-factorisation method to reduce the wrapping effect $\ldots . .19$

2.13 Monotone system with no crossing of trajectories . . . . . . . . 20

2.14 Relation order in the flow of a monotone system . . . . . . . 20

2.15 Examples of graph monotonicity analysis . . . . . . . . . 22

2.16 Example of monotonicity in $\mathbb{R}^{2}$ for cooperative states . . . . . 23

2.17 A partition of the non-monotone states of the set of initial statespace conditions in $\mathbb{R}^{2} \ldots \ldots \ldots \ldots \ldots$

3.1 Diagram of the parallel inputs model . . . . . . . . . . . 26

3.2 Diagram of monotonicity of the parallel inputs model parameters 27

3.3 Diagram of one-compartment equivalent model . . . . . . . 28

3.4 Improvements computing double-peak phenomenon bounds . 31

3.5 Diagram of the parallel inputs model for the Wilinska model . . 31

3.6 Diagram of the parallel inputs model for the extended Wilinska model . . . . . . . . . . . . . . . . 32

3.7 Improvements computing subcutaneous insulin absorption bounds with impulse bolus at $t_{b}=50 \ldots \ldots \ldots \ldots \ldots$

4.1 Diagram of a compartmental in-series model . . . . . . . . . 36 
4.2 Diagram of the linear glucose model developed by Cobelli et al. 41

4.3 Output bounds for the linear glucose model developed by Cobelli et al. . . . . . . . . . . . . . . . . . . 44

4.4 Diagram of the non-linear epidemiological SIS model without disease death rate . . . . . . . . . . . . . . . . 44

4.5 Improvements on the computation of bounds for the proportion of infected individuals . . . . . . . . . . . 45

4.6 Improvements on the computation of bounds for the proportion of infected individuals considering a death by the disease

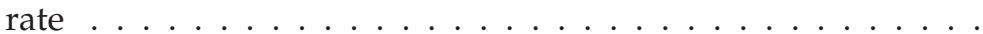

5.1 Under cone monotonicity, a single endpoint of the set of initial state-space conditions does not cover all the space. Example in $\mathbb{R}^{2}$

5.2 Under cone monotonicity, simulations considering the boundary of the set of initial state-space conditions cover all the set of initial state-space conditions. Example in $\mathbb{R}^{2} \ldots \ldots \ldots$

5.3 Under cone monotonicity, when the cone embraces an axis, that subset is reduced to a segment (1-dimension polytope) in $\mathbb{R}^{2}$. . 53

5.4 A partition of the boundary of the set of initial state-space conditions under cone monotonicity in $\mathbb{R}^{2} \ldots \ldots \ldots$. . . . . 54

5.5 Computation of solution envelopes for a non-linear chemostat model ...................... 56

5.6 Computation of solution envelopes for a non-linear chemostat model .....................

6.1 Diagram of the systems interaction . . . . . . . . . . . . 60

6.2 Parameters monotonicity of the carbohydrate digestion and absorption system . . . . . . . . . . . . . . 63

6.3 Parameters monotonicity of the subcutaneous insulin absorption system . . . . . . . . . . . . . . . . . . . 6 64

6.4 Parameters monotonicity of the insulin action system . . . . . . 65

6.5 Parameters monotonicity of the glucose metabolism system . . 67

6.6 Solution envelope obtained for the first scenario . . . . . . . . . 68

6.7 Solution envelope obtained for the second scenario . . . . . . . 68

6.8 Solution envelope obtained for the third scenario . . . . . . . . . 68

6.9 Solution envelope for 30-minute-ahead predictions with glucose measurements every 15 minutes . . . . . . . . . . . 69

7.1 Diagram of the adjustment of uncertain parameters . . . . . . 78

7.2 Diagram of the adjustment of uncertain parameters . . . . . . 79

7.3 Example of in-silico estimations of plasma insulin concentration 81

7.4 Example of real data estimations of plasma insulin concentration 82 


\section{List of Tables}

7.1 Results for in-silico estimation of plasma insulin concentration . 81

7.2 Results for real data estimation of plasma insulin concentration 83

7.3 Results for real data estimation of plasma insulin concentration (excluding the outlier patient) . . . . . . . . . . 83 


\section{1}

\section{Motivation and objectives}

Attentive readers will note numerous holes and contradictions throughout the text; they will even find blatant lies and exaggerations. These have been placed there to test the reader's faith.

Flying Spaghetti Monster

\subsection{Motivation}

We eat food to provide energy to our muscles and other tissues. Most of it is transformed into glucose, a form of sugar that is transported by blood through all the body. Insulin, a peptide secreted from the pancreatic $\beta$-cells, power the absorption of glucose by the cells, reducing the blood glucose concentration, and inhibits glucose production by the liver. Furthermore, glucagon increases the concentration of glucose in blood. Without them, the body would not be able to absorb energy from food [34].

Under physiological conditions, insulin and glucagon are balanced in order to maintain plasma glucose concentration in a narrow range (around 70$140 \mathrm{mg} / \mathrm{dl}$ ). This process is called homeostasis. In the fasting and postabsorptive states, insulin regulates plasma glucose concentration mainly by restraining hepatic glucose production to exactly match peripheral glucose utilization (predominantly by the brain, muscle and adipose tissue). In the postprandial state, the increase in the portal glucose concentration stimulates insulin secretion, which in turn suppresses hepatic glucose production and increases the disposal of the glucose absorbed from the guts [29].

People that suffer diabetes have a malfunction in the production or efficiency of insulin. Patients with Type 1 Diabetes Mellitus suffer an autoimmune destruction of pancreatic $\beta$-cells. As these cells are responsive to secrete 
insulin, their destruction produce a total lack of insulin in the body. As a consequence, people that suffer Type 1 Diabetes have a total dependency on exogenous insulin for survival. In the case of Type 2 Diabetes Mellitus, there is a reduction of the tissues sensitivity to the insulin effects, in conjunction with deficiencies in the production of insulin by the pancreas. This is the most common case of diabetes, and the chances of suffering it increase with obesity, hypertension or elevated cholesterol. As a consequence, the treatment consists in a healthy diet, exercise and some drugs [90].

Diabetes affects to more than 285 million people, around $6.6 \%$ of the total world population. The rate is increasing, as every year around 7 million people develop diabetes. Following this progression, in 20 years around 425 million people will be affected by this disease. Moreover, diabetes also has a high economical impact. In 2002, American Diabetes Association (ADA) estimated the health cost of diabetes in 132 billion dollars just in the United States. Their previsions estimate that it will increase to 192 billion dollars by 2020 [87.

People that suffer any type of diabetes can not obtain efficiently energy from the food they digest. This failure produces an increase in blood glucose concentration, what may lead to severe health problems and chronic complications. Before the discovery of insulin in 1929, this led to fatal acute complications (hyperglycaemia, ketoacidosis, coma and death). Following 1929, thanks to the development of progressively more sophisticated insulin formulations and devices for glucose measurements, acute diabetic complications are rare (although not negligible). However, physiological replacement of insulin secretion remains an elusive goal. This fact results into wide fluctuations of plasma glucose. When the insulin administered is bigger than necessary, blood glucose concentration drops and may produce a life-threatening hypoglycaemia, as the brain will not receive the necessary glucose. On the other hand, if the insulin dose is not enough it may produce chronic hyperglycaemia, being the cause of significant morbidity (end-stage renal disease, blindness, neuropathy) and mortality. Nowadays, there is still no cure for diabetes. Therefore, advances on its treatment may have a great and significant impact.

Insulin therapy in Type 1 Diabetes aims to mimic the pattern of the endogenous insulin secretion found in healthy subjects, trying to avoid the risks of high or low levels of glucose in blood. Patients must try to maintain their blood glucose levels inside a range safe for their health, set between $70 \mathrm{mg} / \mathrm{dl}$ and $140 \mathrm{mg} / \mathrm{dl}$. In order to maintain blood glucose concentration in this range, patients will take a basal injection of insulin to maintain the resting level of the organism. Moreover, at each meal they will follow a three-step process [5]:

Measurement of the current level of glucose. The most common technique to measure blood glucose is to use a glucometer to perform a capillary glucose measurement. This test is performed by taking a drop of blood 
from the finger tip and analysing its glucose concentration. Diabetic patients can perform this measurements at home and by themselves. A controlled patient may perform around 4 measurements per day (one before each meal and another one before going to sleep).

Estimation of the ingested glucose in the meal. After a meal, blood glucose concentration increases. Patients have to estimate the amount of carbohydrates their food has, based on the type of food and on the quantity ingested.

Estimation of the appropriate dosage of exogenous insulin. Insulin doses have to compensate the glucose ingestion to maintain the levels of blood glucose concentration in the adequate range. The most common technique to administer insulin doses is to use a device called pen. The dose can be adjusted in the pen, that has a needle to administer the insulin subcutaneously.

When patients are first diagnosed with diabetes, they are helped by their physicians. However, diabetes treatment is based on autocontrol, as is the patient himself the one who take the decisions in his everyday life based on his previous experiences and periodical indications from his physician. As a consequence, diabetes patients have to be constantly taking care of their disease.

Investigation in diabetes treatment has been fuelled in the last decades following the introduction in the market of portable devices for real-time continuous glucose monitoring. These devices are subcutaneously inserted in the abdomen region. Thus, they offer an estimation of plasma glucose from measurements of interstitial glucose every 5 minutes [75].

New external devices have been also developed for the administration of insulin in a continuous way, known as insulin pumps. These pumps are equipped with a compartment to allocate the insulin and a needle placed in the subcutaneous tissue. They are programmed to administrate a basal dose of insulin continuously through the full day and night. Furthermore, before each meal the patient can also indicate manually the bolus dose of insulin they want to receive, avoiding the use of insulin pens.

Research is now focused on using these devices for the development of an Artificial Pancreas, i.e., a closed-loop glucose control system that automatically dispenses insulin subcutaneously [26]. A closed-loop system is one in which the input is determined by the system response. In this case, this system will take the 'decision' of how much insulin will be administrated at each instant based on the measurements from the continuous glucose monitor and, in some cases, on the food ingested. It would allow diabetic patients to avoid most of the steps mentioned above, as the device would take care of their daily glucose control. As a consequence, it will enhance the quality of life of diabetic patients. 
However, the development of an artificial pancreas is complicated by a large number of uncertainties [7]:

Inter-patient variability. Each patient has a different behaviour based on lifestyle and physiological conditions. For example, insulin absorption may differ around $20-45 \%$ in a clinical environment between patients [36]. Hence, model identification has to be individualized for each patient.

Intra-patient variability. Even the same patient may differ significantly from one day to another. For instance, insulin absorption may vary between 15-25\% in a clinical setting [36]. This uncertainty arises, among other causes, from the different physiological processes involved, such as subcutaneous insulin absorption, circadian rhythms of insulin sensitivity or action of counter-regulatory hormones [84].

Glucose measurement errors. Continuous glucose monitors provide measurements of glucose concentration every 5 minutes. However, the accuracy and reliability of current monitors is still insufficient, although significant advances are being made in this respect.

Meal estimation and variability. In most of artificial pancreas systems, patients have to announce their meals. The amount of carbohydrates ingested is estimated by the patient, introducing a large error [46]. In addition, meal ingestion introduces important disturbances in glucose homeostasis depending, among other things, on the meal composition and even previous meals [69].

\subsection{Objectives}

The aim of this thesis is to provide tools and techniques for the treatment of intra-patient variability and other sources of uncertainty in glycemic control. This problem will be tackled from the development of glycemic predictors that include all the sources of uncertainty. These predictors will compute glucose envelopes that guarantee the inclusion of all the possible behaviours of the patient. The robustness and security of control strategies will be improved with the use of these tools.

Furthermore, there is a lack of awareness about the system itself as it is not possible to measure all its indicators in real time. Only subcutaneous glucose concentration can be measured using a continuous glucose monitor, with some noise on its measurements.

These are the main specific objectives pursued in this thesis:

1. Compute solution bounds for biological models. Model parameters and initial conditions of the most common structures in biological models will be considered as unknown, but bounded by intervals, to com- 
prehend all the variability of real processes. New methods to reduce the overestimation in the computation of solution bounds for these model structures will be proposed.

2. Develop new approaches to compute solution envelopes. Different approaches known in literature to compute solution bounds for systems under uncertainty will be reviewed in the state of the art. New approaches will be analysed that guarantee the inclusion of all the possible solutions, and that reduce the overestimation performed for some systems.

3. Predict glucose concentration under uncertainty. The work developed for the previous objectives will be applied to predict blood glucose concentration under uncertainty. Computed bounds must guarantee the inclusion of all the possible behaviours of the patients, and reduce the overestimation.

4. Estimate plasma insulin concentration in real time. A state observer will be used to estimate insulin concentration based on the meal ingested and on noisy measurements of plasma glucose, given by a continuous glucose monitor. This methodology will be evaluated in-silico and with clinical data from patients with Type 1 diabetes.

\subsection{Outline}

A detailed overview of all the chapters of this thesis is described:

- Chapter 2 starts with a description of the most common models used in Biology and, specifically, in diabetes. It also reviews the different approaches known in literature to compute solution envelopes that guarantee the inclusion of all the possible solutions.

- Common biological model structures are analysed in Chapter 3 (parallel inputs models) and Chapter 4 (in-series models). New techniques are developed to compute guaranteed solution envelopes for both model structures that reduce the overestimation performed.

- In Chapter 5 a new approach to compute guaranteed solution bounds is proposed. This novel method, based on monotonicity with respect to an arbitrary cone, is illustrated by the computation of solution envelopes for a non-linear chemostat model.

- Chapter 6 presents the prediction of plasma glucose concentration under uncertainty. In this chapter, previous results are applied to bound all 
the possible patients behaviours. The computation of guaranteed glucose envelopes has many applications, such as robust model predictive control, robust fault detection or robust parameter identification.

- Chapter 7 describes the use of a state observer to estimate plasma insulin based on the food ingested and on measurements of glucose concentration from a continuous glucose monitor. The glucose-insulin models used in the previous chapter is validated for insulin real-time estimation.

- Finally, the conclusions of this thesis, its contributions and future work are exposed in the last section. 


\section{2}

\section{Simulation of interval dynamic systems}

Le doute n'est pas un état bien agréable, mais l'assurance est un état ridicule.

Voltaire

\subsection{Modelling reality with Mathematics}

Mathematics is amazing by itself, but its real strength is in the fact that it can be used in conjunction with other knowledge fields, such as Biology, Medicine, Engineering, Economics or Human Sciences. Mathematical systems have been widely used to simulate real-life situations. The use of mathematical models can improve the understanding of the processes, and analyse the consequences of taking different decisions.

For example, one of the most famous and relevant applied mathematical systems was developed by Ronald Ross to model the transmission of malaria disease through mosquitoes [15]. Using this model, it was possible to develop effective techniques to fight against the spread of the disease. As a consequence, he received the Nobel Prize for Physiology or Medicine in 1902.

The structure of a mathematical model may vary depending on if the system objective is to mimic the process itself (based on prior knowledge about it) or its behaviour (based on data measurements) [86]:

Phenomenological models are based on prior knowledge about physical or physiological principles. This knowledge determines the structure of the model, while the value of the parameters (speed of the process, degradation, ...) is adjusted based on the observed measurements. This kind of models, also known as knowledge-based models, has a large validity 
domain as long as the structure used is correct. However, they tend to be complex and highly non-linear.

Behavioural models try to approximate the observed behaviour of the output, without any knowledge about the process itself. As a consequence, the parameters of the model may have no concrete meaning at all. These models, focused on data reproduction, are quick and easy to simulate, as no prior knowledge of the phenomenon is needed. However, they can only reproduce similar scenarios to the ones previously shown in the data, restricting the model validity domain.

In the context of the artificial pancreas, behavioural models have been used to characterize diabetic patients behaviour [82]. However, most of the mathematical models published are phenomenological, due to the extensive physiological knowledge available from the physicians. In both cases, the modelling of the glucose-insulin system involves three main sub-processes (represented in Figure 2.1):

Insulin absorption model. Insulin is injected or infused in the subcutaneous tissue, producing a delay in its appearance in plasma. Therefore, this model involves the pharmacokinetics of the insulin diffusion through different tissues, and natural insulin degradation.

Glucose absorption model. The carbohydrates ingested are digested and absorbed from the intestine into blood. The gastrointestinal model represents the catabolisation of the meal ingested to glucose.

Glucoregulatory model. Several physiological processes and relations can affect the concentration of blood glucose, such as the hepatic glucose production or the glucose uptake by peripheral tissues.

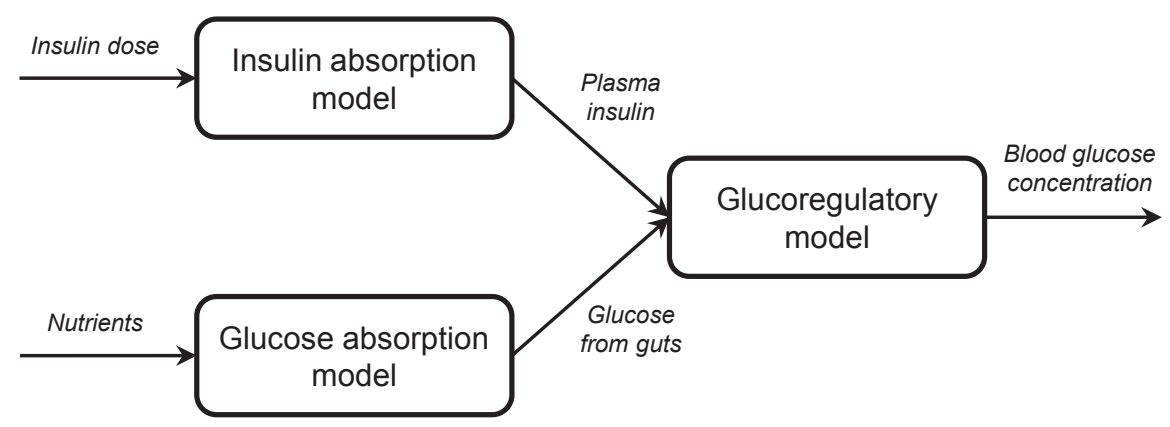

Figure 2.1: Diagram of the glucose-insulin system. 
These processes are usually modelled using compartmental systems, which consist of a finite number of interconnected subsystems called compartments. The interactions among compartments are transfers of material according to the law of conservation of mass [42]. The state variables of these systems represent the amount of material contained in each compartment. For this reason, compartmental systems belong to the broader class of non-negative systems [32].

A common structure in compartmental models is composed of a single linear chain with several compartments connected in series. This structure is known as compartmental in-series models [42]. The compartments may have a physiological meaning (phenomenological models), or they may only have the objective of producing a delay on the output (behavioural models).

In-series models are widely used in insulin and glucose absorption models, as they aim to reproduce the delay in their appearance in plasma. For example, Puckett and Lightfoot developed a phenomenological insulin absorption model [70], in which each compartment represents a physiological region, as shown in Figure 2.2.

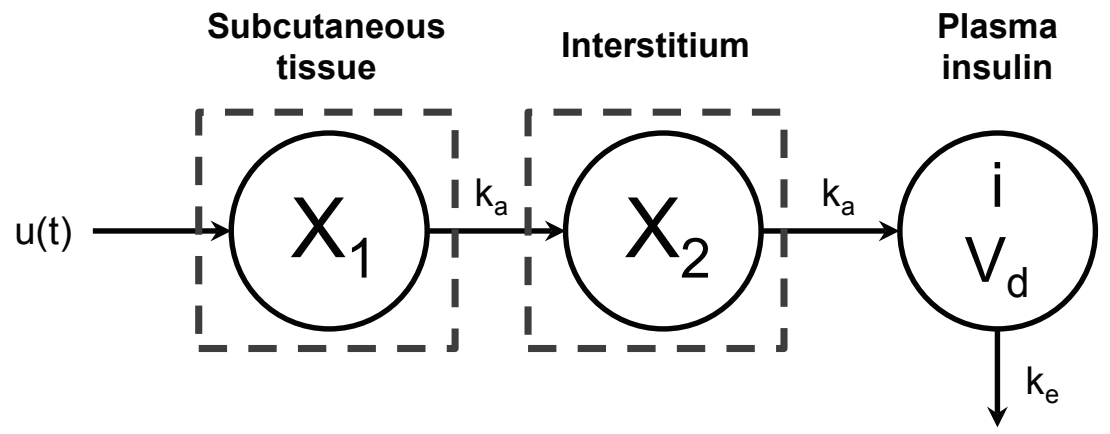

Figure 2.2: Diagram of the phenomenological insulin absorption model developed by Puckett and Lightfoot.

Similar models, but behavioural, were created by Kraegen and Chisholm [49], represented in Figure 2.3. and by Shimoda et al. [78]. In both models, two in-series compartments are used to represent the subcutaneous tissue, producing a delay in the output.

Two or more in-series linear chains can be connected to the output compartment, creating a structure known as parallel inputs model [42]. This model is usually used to mimic processes in which the input shows several effects on the output with different time delays, as each chain may have a different number of compartments. For example, Wilinska et al. use this structure to represent the existence of two channels for the insulin absorption: one of slow absorption and a fast insulin absorption channel [88], represented in Figure 2.4 


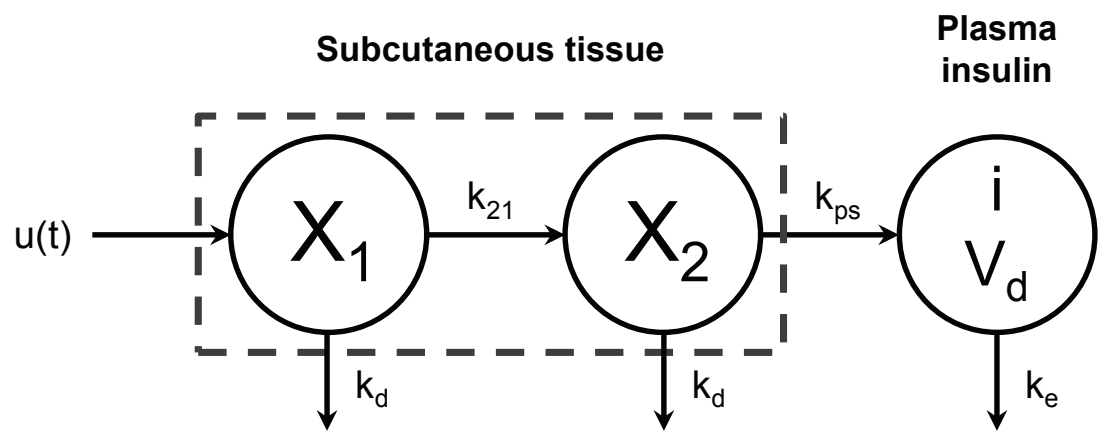

Figure 2.3: Diagram of the behavioural insulin absorption model developed by Kraegen and Chisholm.

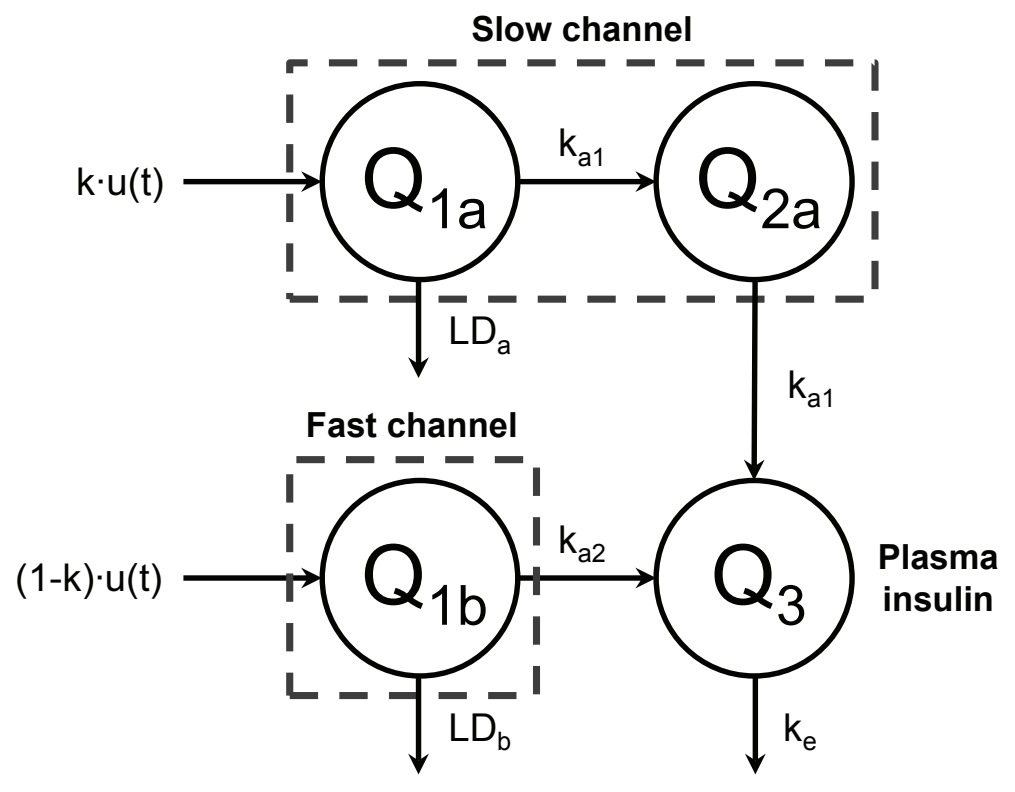

Figure 2.4: Diagram of the two-channel insulin absorption model developed by Wilinska et al.

However, the mathematical models used to reproduce any biological system are usually a simplified version of actual processes, as real-life situations are infinitely complex and they are presented with much variability. This mismatch yields parametric uncertainty and non-modelled dynamics. These uncertainties can be classified in two groups:

Structured uncertainty. In this case, the equations of the model are known, but the values of their parameters are uncertain. 
Unstructured uncertainty. This uncertainty arises due to the lack of information we have about the processes itself. As a consequence, the equations that model the system are uncertain.

In both cases, it is not possible the use of models in which the parameters are known real numbers. Henceforth, uncertainty will be always represented by interval models, in which the parameters, inputs and initial conditions are unknown, but bounded by intervals. The set of intervals $I(\mathbb{R})$ is defined as:

$$
I(\mathbb{R})=\{[a, b] \mid a \in \mathbb{R}, b \in \mathbb{R}, a \leq b\} .
$$

Under known initial condition and parameters values, there is, generally, a single possible solution for the model. However, as interval uncertainty is considered, a set of different possible solutions is achieved, as it is represented in Figure 2.5 .
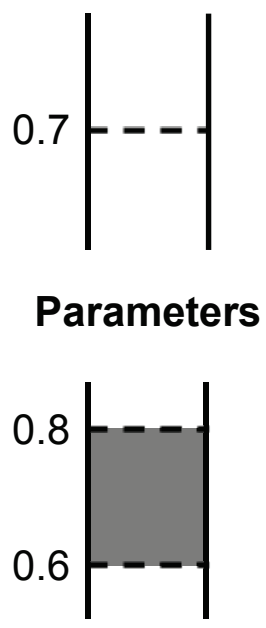
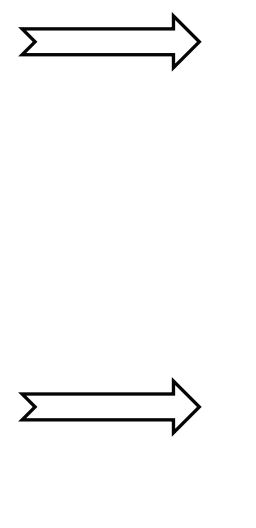

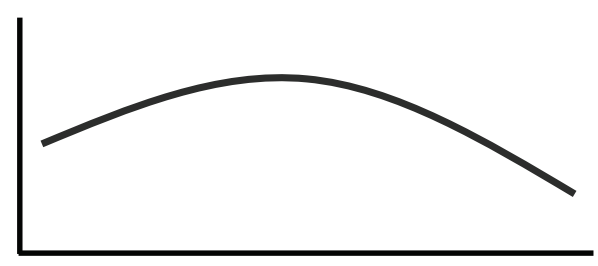

Simulation

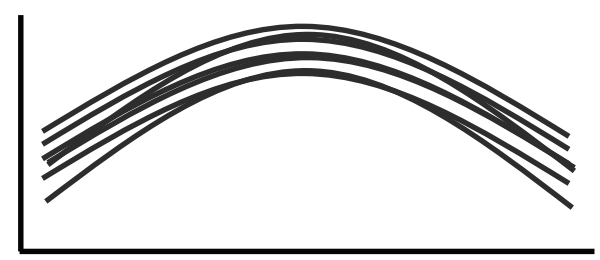

Figure 2.5: Under known parameters values, a single solutions is achieved. When interval uncertainty is considered, a set of possible solutions is obtained.

\subsection{Interval dynamic systems}

A continuous-time system under uncertainty, also known as a continuous interval dynamic system, can be analysed as an initial-value problem (IVP):

$$
\begin{aligned}
& \dot{x}(t, p)=f(t, x, p, u(t)), \quad x\left(t_{0}\right)=x_{0}, \\
& x \in \mathbb{R}^{n}, t \in \mathbb{R}, p \in \mathbb{R}^{n_{p}}, u \in \mathbb{R}^{n}
\end{aligned}
$$


where $f$ is the vector function with components $f_{i}, x$ is the state vector, $p$ is the parameter vector, $n_{p}$ is the number of parameters and $u(t)$ is the input vector. The solution of (2.1) is denoted by $x\left(t ; t_{0}, x_{0}, p, u\right)$.

Representing intervals in bold, interval vectors $\mathbf{p}, \mathbf{u}(t)$ and $\mathbf{x}_{\mathbf{0}}$ include all possible values for the parameters $p$, for the input vector $u(t)$ and for the initial conditions $x_{0}$ of the model, respectively. The set of possible solutions derived from parametric uncertainty is denoted by $\mathbf{x}\left(t ; t_{0}, \mathbf{x}_{\mathbf{0}}, \mathbf{p}, \mathbf{u}(t)\right)$ :

$$
\mathbf{x}\left(t ; t_{0}, \mathbf{x}_{\mathbf{0}}, \mathbf{p}, \mathbf{u}(t)\right)=\left\{x\left(t ; t_{0}, x_{0}, p, u(t)\right) \mid x_{0} \in \mathbf{x}_{\mathbf{0}}, p \in \mathbf{p}, u(t) \in \mathbf{u}(t)\right\} .
$$

Several methods are used to analyse the the behaviour of this set.

\subsubsection{Monte Carlo simulation}

Monte Carlo methods date from the 1940s, and they are still a popular method to represent the possible behaviours of a model simulation. These methods consist in performing a large number of different simulations by the variation of the initial states and parameters values, following a probability distribution [35]. In this work, interval parameters and initial conditions are represented in terms of uniform probability distributions. Hence, they assume any value inside the interval with equal probability. The probability density function of the continuous uniform distribution $U(a, b)$ is represented in Figure 2.6 and it is given by:

$$
f(x)=\left\{\begin{array}{cl}
\frac{1}{b-a} & \text { if } a \leq x \leq b \\
0 & \text { otherwise }
\end{array}\right.
$$

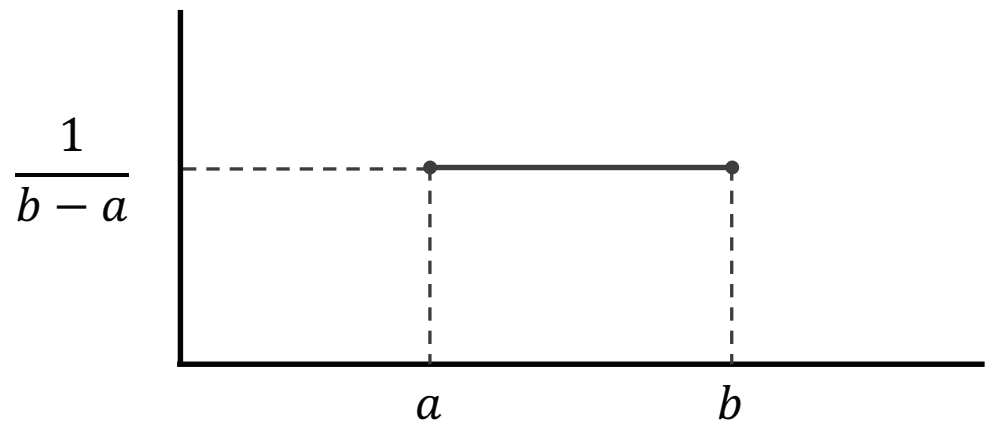

Figure 2.6: Probability density function of the continuous uniform distribution.

These methods have been widely used to deal with uncertainty due to their easy computation. They can even be used in situations that are unfeasible or impossible to compute with deterministic algorithms. 
However, the computational cost of Monte Carlo methods increases proportionally to the number of simulations performed (in the order of thousands or even millions) to cover the uncertain input space sufficiently. Moreover, they are not applicable for computing output bounds because, independently of the number of simulations executed, the bounds obtained do not guarantee the inclusion of all the possible solutions [11].

\subsubsection{Guaranteed solution envelopes}

When a model is used as part of a medical decision system, as is the case in most of the examples of this thesis, the output bounds must ensure that all the possible responses by the patient are outside of risk levels [11]. Moreover, this robust prediction is a necessity for several domains:

Robust model predictive control. In Model Predictive Control (MPC), a model is used to predict the future evolution of the process to optimize the control signal. Robust MPC is able to account for set bounded disturbance while still ensuring that state constraints are met [10].

Robust fault detection aims to identify when a fault has occurred, checking the consistency between the system's behaviour (obtained from the observations) and the model's behaviour [1].

Robust parameter identification consists of finding error-bounded approximations for the set of parameters of a given model that are consistent with the measurements [12].

Interval observers compute guaranteed intervals for the unmeasured variables (or parameters) of an uncertain dynamical system. This estimation is based on uncertainty intervals and on noisy measurements [31].

Constraint-satisfaction problems like diabetes, as blood glucose concentration must be inside a safe range. Insulin pump therapies must satisfy this constraint [73].

Robust insulin dosage optimisation calculates a safer prediction of possible hyper- and hypoglycemia episodes, based on blood glucose prediction under uncertainty [26].

Algorithms for computing guaranteed output bounds can be classified as:

Modal interval analysis. A structural, algebraic, and logical completion of the classical intervals is defined, where a quantifier is attached to a classical interval [27].

Region-based approaches. The output bounds computation is performed using one-step-ahead iteration based on previous approximations of the reachable set [71]. 
Trajectory-based approaches. A set of point-wise trajectories is generated by selecting particular values of the parameters $p \in \mathbf{p}$, the input vector $u(t) \in \mathbf{u}(t)$, and the initial conditions $x_{0} \in \mathbf{x}_{\mathbf{0}}$ using heuristics or optimisation [71].

\subsubsection{Modal interval analysis}

A modal interval is defined as a pair $X=\left(X^{\prime}, \forall\right)$ or $X=\left(X^{\prime}, \exists\right)$, where $X^{\prime}$ is a classical interval that belongs to $I(\mathbb{R})$, and the quantifiers $\forall$ and $\exists$ are a selection modality. The set of modal intervals is denoted by $I^{*}(\mathbb{R})$ [27, 77]:

$$
I^{*}(\mathbb{R})=\left\{\left(X^{\prime},\{\exists, \forall\}\right) \mid X^{\prime} \in I(\mathbb{R})\right\}
$$

Modal intervals of the type $X=\left(X^{\prime}, \exists\right)$ are called proper intervals, while modal intervals $A=\left(X^{\prime}, \forall\right)$ are called improper intervals. The canonical notation of modal intervals is represented in Figure 2.7 and it is introduced by the following definitions:

- Proper interval: $\quad X=[a, b]=\left([a, b]^{\prime}, \exists\right) \quad$ if $\quad a \leq b$

- Improper interval: $\quad X=[a, b]=\left([b, a]^{\prime}, \forall\right)$ if $a \geq b$

- Point-wise interval: $\quad X=[a, b]=\left([a, b]^{\prime},\{\exists, \forall\}\right)$ if $\quad a=b$

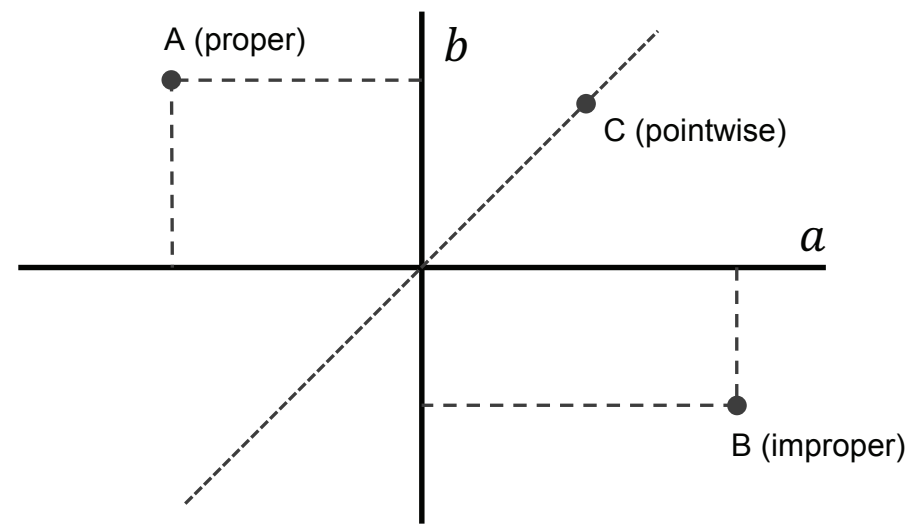

Figure 2.7: Diagram of proper and improper intervals.

where the point-wise interval $[a, a]$, also represented as $[a]$, is a proper and a improper interval.

Example 2.2.1. The proper interval $[2,3]$ corresponds to $\left([2,3]^{\prime}, \exists\right)$, while the improper interval $[3,2]$ corresponds to $\left([2,3]^{\prime}, \forall\right)$. 
Given an interval, the operators Prop, Impr and Dual are defined as

$$
\begin{gathered}
\operatorname{Prop}([a, b])= \begin{cases}{[\mathrm{a}, \mathrm{b}]} & \text { if } a \leq b, \\
{[\mathrm{~b}, \mathrm{a}]} & \text { if } a>b,\end{cases} \\
\operatorname{Impr}([a, b])= \begin{cases}{[\mathrm{b}, \mathrm{a}]} & \text { if } a \leq b, \\
{[\mathrm{a}, \mathrm{b}]} & \text { if } a>b,\end{cases} \\
\operatorname{Dual}([a, b])=[b, a] .
\end{gathered}
$$

Example 2.2.2. Given a modal interval $[-2,3]$, the operators Prop, Impr and Dual are:

$$
\begin{gathered}
\operatorname{Prop}([-2,3])=\operatorname{Prop}([3,-2])=[-2,3], \\
\operatorname{Impr}([-2,3])=\operatorname{Impr}([3,-2])=[3,-2], \\
\text { Dual }([-2,3])=[3,-2]
\end{gathered}
$$

The inclusion among modal intervals is defined as follows

$$
\left[a_{1}, a_{2}\right] \subseteq\left[b_{1}, b_{2}\right] \Longleftrightarrow\left(a_{1} \geq b_{1}, a_{2} \leq b_{2}\right),
$$

that satisfies the condition $A \subseteq B \Longleftrightarrow \operatorname{Dual}(A) \supseteq \operatorname{Dual}(B)$.

Example 2.2.3. Given the intervals $A=[2,4]=\left([2,4]^{\prime}, \exists\right)$ and $B=[4,2]=$ $\left([2,4]^{\prime}, \forall\right)$ :

$$
B \subseteq A \Longleftrightarrow[4,2] \subseteq[2,4] .
$$

The less or equal relation is defined as

$$
\left[a_{1}, a_{2}\right] \leq\left[b_{1}, b_{2}\right] \Longleftrightarrow\left(a_{1} \leq b_{1}, a_{2} \leq b_{2}\right) .
$$

The pair $(\subseteq, \leq)$ is represented in Figure 2.8 .

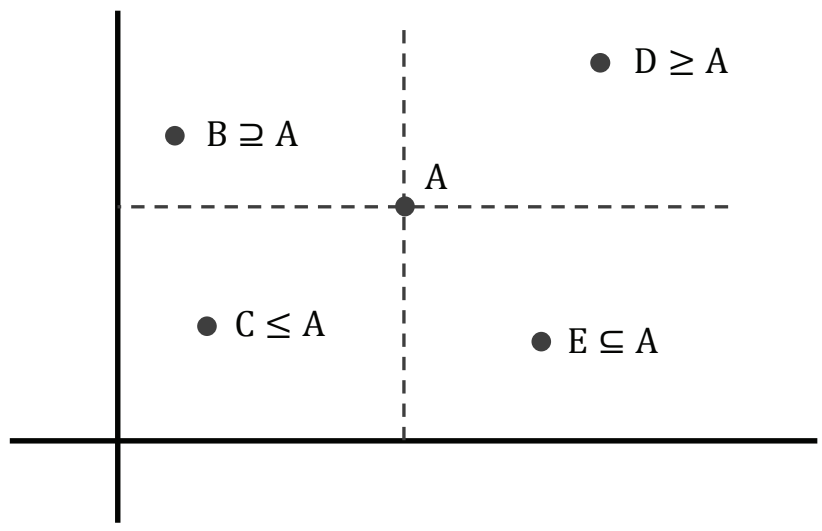

Figure 2.8: Diagram of inclusion and 'less than' relations. 
The operator meet $(\wedge)$ for modal intervals is defined as the $\subseteq$-maximum interval contained in both intervals, while the operation join $(\vee)$ is defined as the $\subseteq$-minimum interval which contains both intervals. Given $A=\left[a_{1}, a_{2}\right]$ and $B=\left[b_{1}, b_{2}\right]$,

$$
\begin{aligned}
& A \wedge B=\left[\max \left\{a_{1}, b_{1}\right\}, \min \left\{a_{2}, b_{2}\right\}\right], \\
& A \vee B=\left[\min \left\{a_{1}, b_{1}\right\}, \max \left\{a_{2}, b_{2}\right\}\right] .
\end{aligned}
$$

Example 2.2.4. Given two modal intervals $A=[2,4]$ and $B=[7,5]$,

$$
\begin{aligned}
& A \wedge B=[2,4] \wedge[7,5]=[7,4] \\
& A \vee B=[2,4] \vee[7,5]=[2,5] .
\end{aligned}
$$

The operators Min and Max for modal intervals are defined as

$$
\begin{aligned}
\operatorname{Min}\{A, B\} & =\left[\min \left\{a_{1}, b_{1}\right\}, \min \left\{a_{2}, b_{2}\right\}\right], \\
\operatorname{Max}\{A, B\} & =\left[\max \left\{a_{1}, b_{1}\right\}, \max \left\{a_{2}, b_{2}\right\}\right] .
\end{aligned}
$$

These operators are represented in Figure 2.9
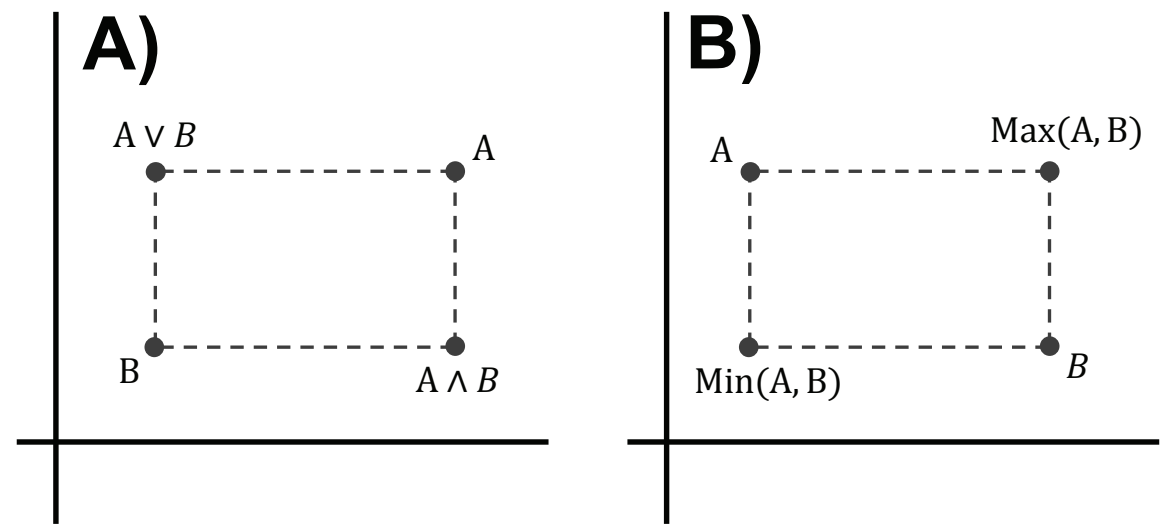

Figure 2.9: Diagram of lattice operators: A) Meet and Join. B) Max and Min.

The modal interval arithmetic includes four basic operations: sum, rest, product and division.

$$
\begin{aligned}
& \text { Sum : } A+B=\left[a_{1}, a_{2}\right]+\left[b_{1}, b_{2}\right]=\left[a_{1}+b_{1}, a_{2}+b_{2}\right] \\
& \text { Rest : } A-B=\left[a_{1}, a_{2}\right]-\left[b_{1}, b_{2}\right]=\left[a_{1}-b_{2}, a_{2}-b_{1}\right]
\end{aligned}
$$

For the sake of brevity, the product and division operations are omitted. For a further understanding of modal interval analysis, the reader is referred to Gardeñes et al. [27] and Sainz et al. [77]. Modal interval can be applied to compute solution envelopes that guarantee the inclusion of all the possible solutions [11]. 
However, some overestimation is produced due to the multi-incidence problem [60], as multiple instances of the same variable appear on the system. This phenomenon can be easily understood if $x-x$ is computed with interval arithmetic, where $x \in[-1,1]$. The result should be 0 , but due to the multi-incidence problem it is $[-2,2]$. A similar effect is produced when some states or parameters appear with opposite effects in different equations, or even in the same equation.

\subsubsection{Region-based approaches}

Region-based approaches are performed in two phases [45, 61], and applying one-step-ahead iteration based on previous approximations of the reachable set. The first step consists in finding an a priori enclosure $\tilde{\mathbf{x}}_{\mathbf{i}}$ for an interval $\left[t_{i}, t_{i+1}\right]$, supposing $\mathbf{x}_{\mathbf{i}}$ has been computed at $t_{i}$ such that

$$
\mathbf{x}\left(t_{i} ; t_{0}, \mathbf{x}_{\mathbf{0}}, \mathbf{p}, \mathbf{u}(t)\right) \subseteq \mathbf{x}_{\mathbf{i}} .
$$

There is a unique solution of the state vector $\mathbf{x}(t)$ for each $x_{i} \in \mathbf{x}_{\mathbf{i}}, t \in$ $\left[t_{i}, t_{i+1}\right]$, such that

$$
\mathbf{x}\left(t ; t_{i}, \mathbf{x}_{\mathbf{i}}, \mathbf{p}, \mathbf{u}(t)\right) \subseteq \tilde{\mathbf{x}}_{\mathbf{i}} \quad \forall t \in\left[t_{i}, t_{i+1}\right]
$$

The second step uses $\tilde{\mathbf{x}}_{\mathbf{i}}$ to enclose the truncation error of the method and computes a tighter enclosure $\mathbf{x}_{\mathbf{i}+\mathbf{1}}$ at $t_{i+1}$ such that

$$
\mathbf{x}\left(t_{i+1} ; t_{0}, \mathbf{x}_{\mathbf{0}}, \mathbf{p}, \mathbf{u}(t)\right) \subseteq \mathbf{x}_{\mathbf{i}+\mathbf{1}} \subseteq \tilde{\mathbf{x}}_{\mathbf{i}} .
$$

Figure 2.10 represent bounds computed in these two phases.

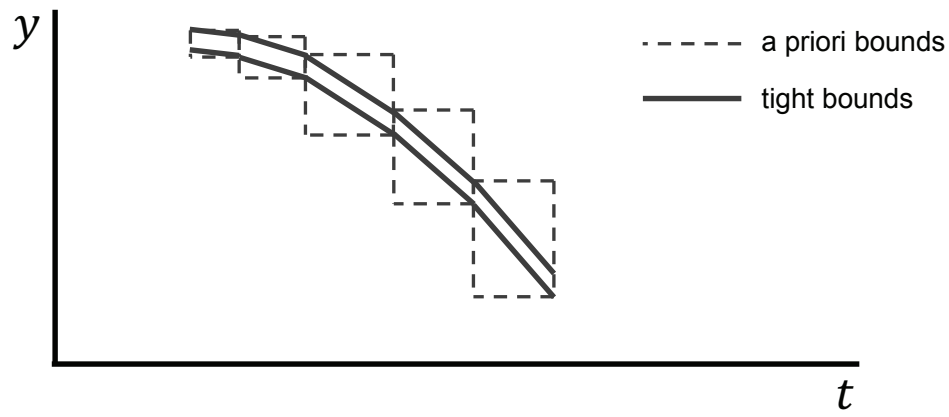

Figure 2.10: Representation of a priori and tight bounds.

In contrast to traditional ODE solvers, which compute approximate solutions, region-based solvers prove that a unique solution for the problem exists; afterward rigorous bounds that guarantee the enclosure of the solution are computed. 
At each iteration, the true solution set is wrapped into a region-based on outer approximations. This produces an overestimation commonly known as the wrapping effect [61, 71]. Regions must be feasible to be constructed and represented on a computer, or the region representation will produce a significant overestimation. The errors involved can quickly accumulate, and hence, the solution envelope of the interval system explodes, as it is represented in Figure2.11.
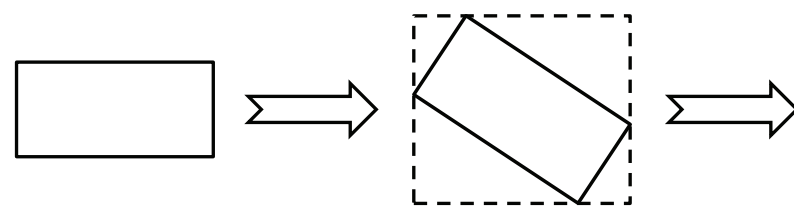

Figure 2.11: Representation of the wrapping effect.

Several methods have been proposed to avoid the wrapping effect, or at least to reduce it, since it was first observed in the early 1960s [60]. These methods include:

A change of coordinates. Moore [60] proposes to perform a change of coordinates to reduce the overestimation in the interval hull of the reachable set.

QR-factorisation. This approach puts a box in a moving orthogonal coordinate system, in order to match this box with one of the edges of the enclosed parallelepiped. Intuitively, if the box matches the longest edge the overestimation will be smaller, as represented in Figure 2.12 This method, introduced by Lohner [56], is an evolution of Moore's algorithm.

Ellipsoids. Instead of using an interval hull of the reachable set, Neumaier [65] proposes a method, based on interval ellipsoid arithmetic, to compute the smallest ellipsoid that include the reachable set.

Zonotopes. Kühn [50] introduces the idea of using zonotopes to define the reachable set. Zonotopes are polytopes (geometric objects with flat sides) with symmetry under rotations through 180 degrees.

Hermite-Obreschkoff method. Nedialkov [64] proposes an algorithm based on Taylor series that consist on two phases: a predictor and a corrector. The predictor computes an initial enclosure of the solution, while the corrector applies Newton-like step to tighten the initial enclosure. 


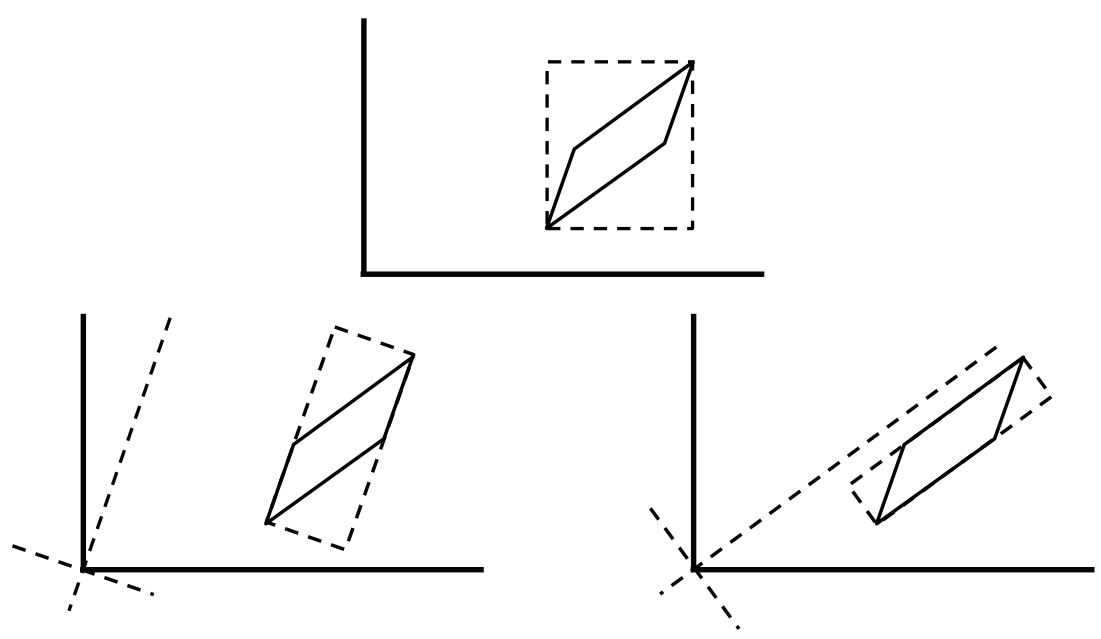

Figure 2.12: QR-factorisation method to reduce the wrapping effect.

Several interval solvers have been developed to compute guaranteed solution bounds for an interval IVP following a region-based approach. This software allows the development of numerical applications using interval arithmetic.

AWA. Software package for verified solution of ordinary differential equations developed by Lohner [57].

COSY. Based on Taylor model and interval methods [58]. It is intended to compute verified solutions of problems such as ordinary differential equations, quadrature or range bounding.

VNODE. $\mathrm{C}++$ based Interval Solver for Initial Value Problems in Ordinary Differential Equations [63].

VNODE-LP. This package is a successor of the VNODE package. A distinctive feature of the VNODE-LP solver [62] is that it is implemented entirely using literate programming [48]. As a result, its correctness can be verified by a human expert, similarly to how mathematical results are certified.

ValEncIA-IVP. Software for VALidation of state ENClosures using Interval Arithmetic for Initial Value Problems [2].

\subsubsection{Trajectory-based approaches}

Trajectory-based approaches consist in performing a monotonicity analysis of the states and parameters of the model. Monotone systems have very robust 
dynamical characteristics, since they respond to perturbations in a predictable way. Figure 2.13 represents a monotone system given by $\dot{x}(t)=-0.5 x+1$ and initial conditions $x(0)=[0,1]$, whose trajectories do not cross, as the relation order is kept.

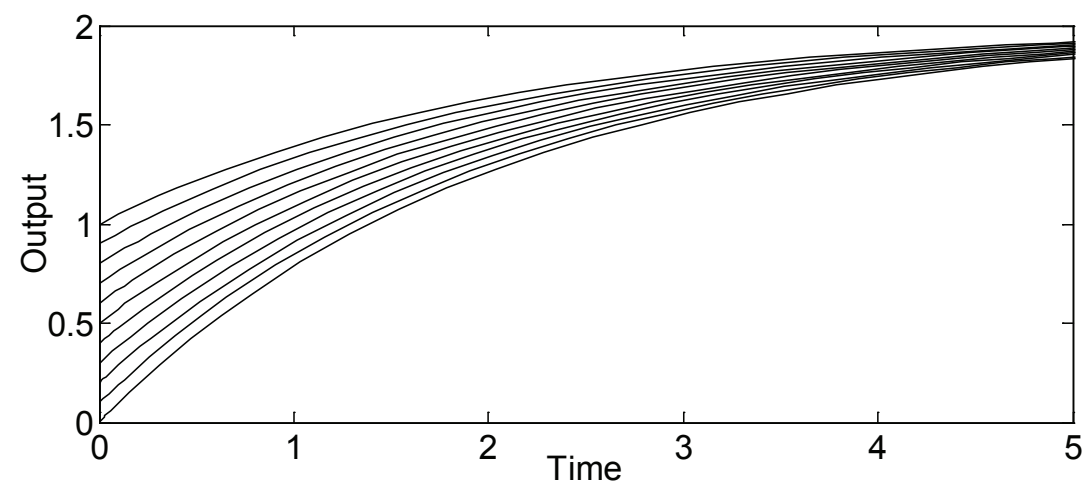

Figure 2.13: Monotone system given by $\dot{x}(t)=-0.5 x+1$ and initial conditions $x(0)=[0,1]$ without crossing of trajectories.

The interconnection of monotone systems may be studied by considering a flow $\mathbf{x}(t)=\phi\left(\mathbf{x}_{\mathbf{0}}, t\right)$. A system is monotone if

$$
\mathbf{x}_{\mathbf{0}} \preceq \mathbf{y}_{\mathbf{0}} \Rightarrow \phi\left(\mathbf{x}_{\mathbf{0}}, t\right) \preceq \phi\left(\mathbf{y}_{\mathbf{0}}, t\right)
$$

for all $t \geq 0$, where $\preceq$ is a given relation order [45], as represented in Figure 2.14

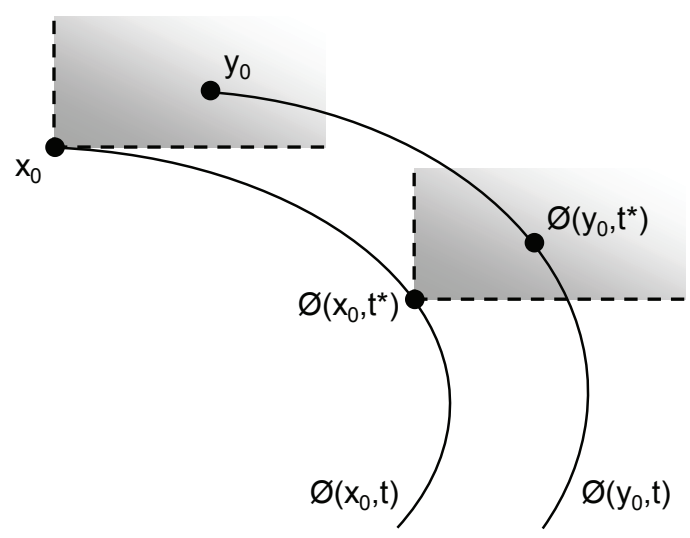

Figure 2.14: Relation order in the flow of a monotone system. 
Monotone systems may be also studied in an analytical way [45], where the rate of change of each state is expressed as a function of the rest of states at that time:

$$
\begin{aligned}
\dot{x}_{1}(t, p) & =f_{1}\left(t, x_{1}(t), \ldots, x_{n}(t), p_{1}, \ldots, p_{n_{p}}, u(t)\right) \\
\dot{x}_{2}(t, p) & =f_{2}\left(t, x_{1}(t), \ldots, x_{n}(t), p_{1}, \ldots, p_{n_{p}}, u(t)\right) \\
& \vdots \\
\dot{x}_{n}(t, p) & =f_{n}\left(t, x_{1}(t), \ldots, x_{n}(t), p_{1}, \ldots, p_{n_{p}}, u(t)\right)
\end{aligned}
$$

simplified by $\frac{d \mathbf{x}}{d t}=\mathbf{f}(\mathbf{x}, \mathbf{p}, \mathbf{u}(t))$, where $\mathbf{f}$ is the vector function with states $f_{i}$. A system is monotone if:

$$
\frac{\partial f_{i}}{\partial x_{j}} \cdot \frac{\partial f_{j}}{\partial x_{i}} \geq 0, \quad \text { for all } i \neq j, t \in \mathbb{R}^{+} .
$$

Moreover, cooperative systems [79] form a class of monotone dynamical systems in which

$$
\frac{\partial f_{i}}{\partial x_{j}} \geq 0, \quad \text { for all } i \neq j, t \geq 0 .
$$

The monotonicity of the system with respect to the parameters of the model can be analysed by considering the parameters as system states in an extended model [71], that is, by performing a monotonicity analysis of a new system with $n+n_{p}$ states given by:

$$
\begin{aligned}
\dot{x}_{1}(t) & =f_{1}\left(t, x_{1}(t), \ldots, x_{n}(t), p_{1}(t), \ldots, p_{n_{p}}(t), u(t)\right) \\
& \vdots \\
\dot{x}_{n}(t) & =f_{n}\left(t, x_{1}(t), \ldots, x_{n}(t), p_{1}(t), \ldots, p_{n_{p}}(t), u(t)\right) \\
\dot{p}_{i}(t) & =0 \quad \forall i \in\left\{1, \ldots, n_{p}\right\}
\end{aligned}
$$

Thus, a system 2.1 is monotone with respect to a parameter if the extended system (2.2) is monotone with respect to the corresponding extended model states.

Graph theory also allows analysing monotone and cooperative systems by using a species graph [81], in which a node is assigned for each state or parameter of the model. If the node $x_{i}$ has no direct effect on node $x_{j}$, the partial derivative $\frac{\partial f_{j}}{\partial x_{i}}(x)$ equals zero; thus no edge is drawn from node $x_{i}$ to node $x_{j}$. If the effect of the node $x_{i}$ on node $x_{j}$ is positive, the derivative is strictly positive, an activation arrow $(\rightarrow)$ is drawn. Finally, if the effect is negative, an inhibition line $(-)$ is drawn. However, if the derivative sign changes depending on the particular entries, both an activation arrow and an inhibition line are drawn from node $x_{i}$ to node $x_{j}$.

A spin assignment is an allocation in which each node has a sign, such that nodes connected by an activation arrow $(\rightarrow)$ have the same sign, while nodes connected by an inhibition line $(-1)$ have different signs. If at least one 
consistent assignment exists, the dynamical system is monotone. Moreover, if all nodes are connected by activation arrows $(\rightarrow)$, the system is cooperative.Figure 2.15 shows an example of a monotone system and a non-monotone system.
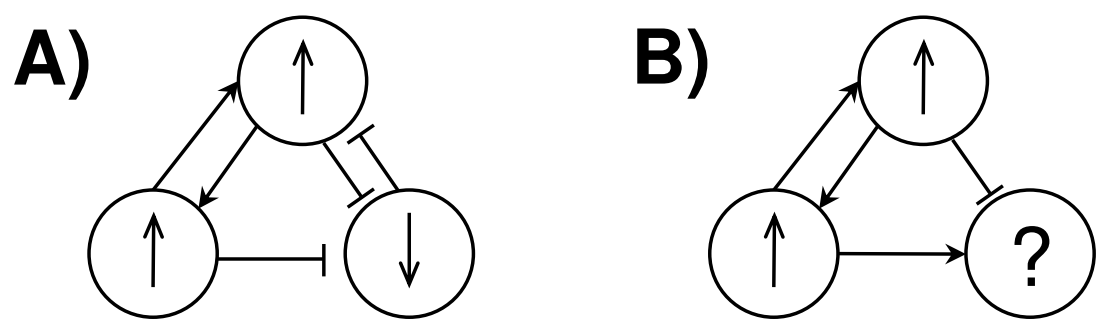

Figure 2.15: Examples of graph monotonicity analysis. A) A monotone system. B) A nonmonotone system.

Therefore, the states and parameters of a system can be classified [81] as:

Cooperative. They have a positive effect on the output of the system. If their value is increased, the output will increase (or it will remain the same). On the other hand, if they are decreased, the output will decrease.

Competitive. They are monotone but non-cooperative states or parameters, as they have a negative effect on the system. If their value increases or decreases, the output will decrease or increase, respectively.

Non-monotone. They are generally produced due to a multi-incidence problem [60. Their effect on the output is unknown, or may vary from a positive to a negative effect depending on several factors.

In order to calculate output bounds, two models are considered: an upper bounding model and a lower bounding model. In an upper bounding model, cooperative states and parameters with respect to the solution take their maximum values, while competitive states and parameters take their minimum values. On the other hand, a lower bounding model is obtained by considering the minimum value of cooperative states and parameters, and the maximum value of competitive states and parameters. Despite parametric uncertainty, only interval endpoints of monotone states and parameters (cooperative or competitive) are needed for the computation of the upper and the lower output bounds, as seen in Figure 2.16

In the upper bounding model and in the lower bounding model, nonmonotone states and parameters are still considered as intervals, and these interval uncertainties will produce an overestimation on the computation of output bounds. 

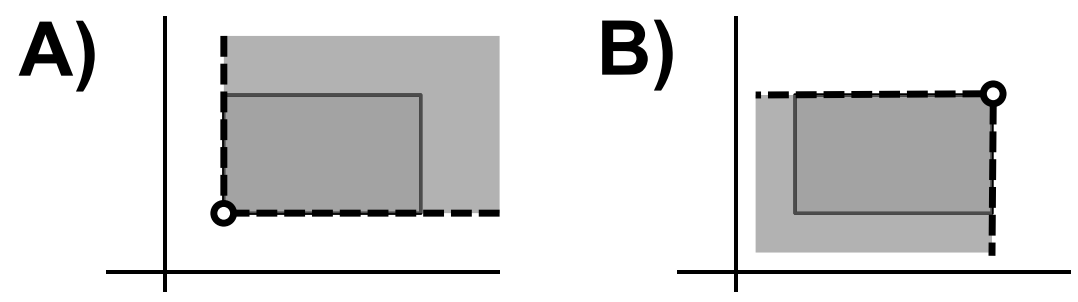

Figure 2.16: Example of monotonicity in $\mathbb{R}^{2}$ for cooperative states. A) Value for the lower bound computation. B) Value for the upper bound computation.

\subsubsection{Partition of the input space}

In order to reduce the overestimation produced by the non-monotone states, Moore [60] proposes to partition the input space $R$ into smaller spaces $\mathcal{R}_{i}$, such that

$$
R=\bigcup_{i} \mathcal{R}_{i} \quad \text { where } \mathcal{R}_{i} \in \mathbb{R}^{n} \text { and } \mathcal{R}_{i} \cap \mathcal{R}_{j}=\emptyset, i \neq j
$$

as represented in Figure 2.17
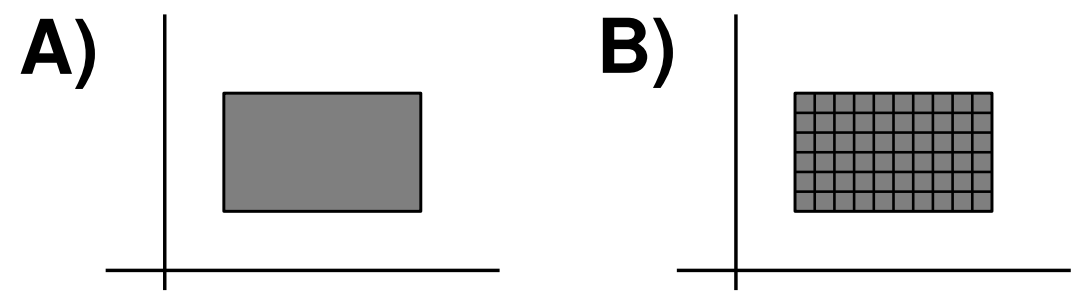

Figure 2.17: A partition of the non-monotone states of the set of initial state-space conditions in $\mathbb{R}^{2}$. A) Set of initial state-space conditions. B) Partition of the set of initial state-space conditions.

Now, defining the lower and the upper trajectories generated by the set of initial state-space conditions $R_{i}$ for each time $t$ as

$$
\begin{aligned}
& x_{\text {inf }}\left(t ; t_{0}, R_{i}, p, u(t)\right)=\inf \left\{x\left(t ; t_{0}, y_{0}, p, u(t)\right) \mid y_{0} \in R_{i}\right\}, \\
& x_{\text {sup }}\left(t ; t_{0}, R_{i}, p, u(t)\right)=\sup \left\{x\left(t ; t_{0}, y_{0}, p, u(t)\right) \mid y_{0} \in R_{i}\right\},
\end{aligned}
$$

as well as the lower and the upper trajectories given by $R$ as

$$
\begin{aligned}
& x_{\text {inf }}\left(t ; t_{0}, R, p, u(t)\right)=\inf \left\{x_{\text {inf }}\left(t ; t_{0}, R_{i}, p, u(t)\right) \mid R_{i} \subset R\right\}, \\
& x_{\text {sup }}\left(t ; t_{0}, R, p, u(t)\right)=\sup \left\{x_{\text {sup }}\left(t ; t_{0}, R_{i}, p, u(t)\right) \mid R_{i} \subset R\right\},
\end{aligned}
$$

then the set of possible solutions can be bounded: 


$$
x_{\text {inf }}\left(t ; t_{0}, R, p, u(t)\right) \leq x\left(t ; t_{0}, x_{0}, p, u(t)\right) \leq x_{\text {sup }}\left(t ; t_{0}, R, p, u(t)\right) \quad \forall x_{0} \in R .
$$

This method can be applied in conjunction with any of the previous approaches, with the objective of reducing the overestimation. However, the computational cost increases drastically with the number of partitions. 


\section{3}

\section{Solution envelopes for parallel inputs models}

I just wanted to write something about running, but I realized that to write about my running is to write about my writing. It's a parallel thing in me.

Haruki Murakami

\subsection{Introduction}

Parallel inputs models are a common structure in Biology to represent several effects from the same input with different time delays, as seen in Section 2.1 The aim of this chapter is to compute tight solution envelopes for parallel inputs models that guarantee the inclusion of all possible behaviours of this system.

Solution bounds have been computed following state-of-the-art methods. First, a region-based approach has been performed using VNODE-LP software [62], trying to avoid the wrapping effect. Moreover, a monotonicity analysis (trajectory-based approach) has been performed to compute guaranteed solution envelopes. However, if the model includes non-monotone compartments or parameters, the computation of such a solution envelope may produce a significant overestimation.

A new method is proposed in which a model reduction is performed in conjunction with analytical solutions of the input chains and a monotonic-

The work in this chapter has been partially published in the paper On the computation of output bounds on parallel inputs pharmacokinetic models with parametric uncertainty, published by Diego De Pereda, Sergio Romero-Vivo and Jorge Bondia in Mathematical and Computer Modelling 57, 7 (2013), 1760-1767. 
ity analysis of model states and parameters. This method allows obtaining tighter output bounds with low computational cost compared to the current techniques. The proposed method has been applied to two cases: the study of the double-peak phenomenon observed for certain drugs, and subcutaneous insulin absorption for artificial pancreas research.

\subsection{Parallel inputs model}

This chapter is focused on a parallel inputs model [42], which is based on a suggestion given by Jacquez, who considered that the single-peak concentrationtime response that usually follows the oral administration of a drug could be modelled using a single linear chain of compartments connected together in series. A similar approach is based on two or more parallel linear chains connected to the output compartment, represented by $x_{f}$, with elimination rate $e_{f}$. Each chain is formed by a number (that can vary for each chain) of compartments with identical transfer rates, as shown in Figure 3.1

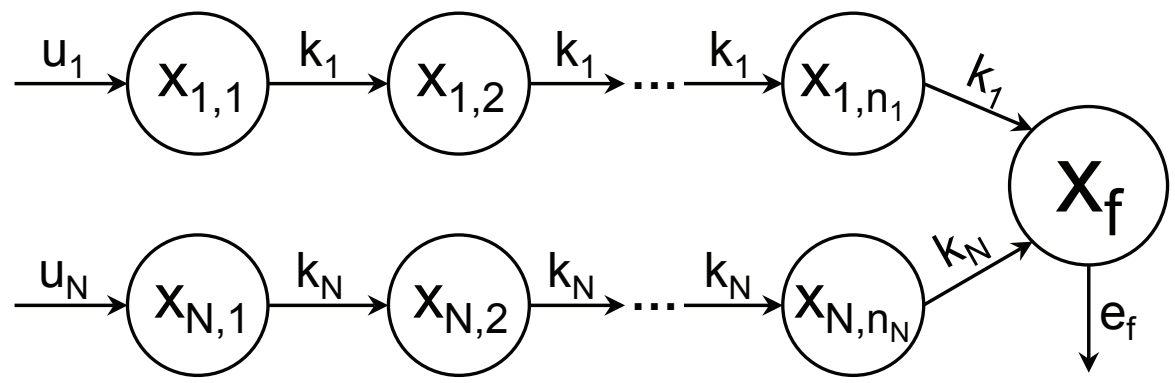

Figure 3.1: Diagram of the parallel inputs model.

Each chain produce a different delay in the input effect, characterized by the number of compartments and the absorption rates of each chain. Longer chains are used for representing larger delays.

The inputs of each chain can be considered as impulses, continuous functions, or both. As a general case, each chain has an impulsive input $D_{i} \delta(t)$, where $D_{i}=x_{i, 1}(0)$ is the initial condition, and a continuous input $u_{i}$. Each chain is formed by $n_{i}$ compartments, joined with the same absorption rate $k_{i}$.

The differential equations for the parallel inputs model composed of $N$ chains are, for $i=1, \ldots, N$, 


$$
\begin{aligned}
\dot{x}_{i, 1} & =u_{i}-k_{i} x_{i, 1} & x_{i, 1}(0) & =D_{i} \\
\dot{x}_{i, 2} & =k_{i} x_{i, 1}-k_{i} x_{i, 2} & x_{i, 2}(0) & =0 \\
\dot{x}_{i, 3} & =k_{i} x_{i, 2}-k_{i} x_{i, 3} & x_{i, 3}(0) & =0 \\
& \vdots & & \vdots \\
\dot{x}_{i, n_{i}} & =k_{i} x_{i,\left(n_{i}-1\right)}-k_{i} x_{i, n_{i}} & x_{i, n_{i}}(0) & =0 \\
\dot{x}_{f} & =\sum_{i=1}^{N} k_{i} x_{i, n_{i}}-e_{f} x_{f} & x_{f}(0) & =x_{f, 0}
\end{aligned}
$$

One of the advantages of the parallel inputs model is that, despite the large number of compartments $\left(1+\sum_{i=1}^{N} n_{i}\right)$ that are included in the model, there are a few inputs $\left(u_{i}\right)$, parameters $\left(k_{i}\right.$ and $\left.e_{f}\right)$ and initial conditions $\left(D_{i}\right.$ and $\left.x_{f, 0}\right)$ under uncertainty $(3 N+2)$.

Using graph theory to perform a monotonicity analysis, Figure 3.2 shows a spin assignment of the parallel inputs model in which the parameters are considered as extended states of the system. The compartments $x_{f}$ and $x_{i, j}$ are cooperative; thus the initial conditions $D_{i}$ and $x_{f, 0}$ are also cooperative, according to the definitions given in Section 2.2.5. Furthermore, the parameters $u_{i}$ are cooperative, while the parameter $e_{f}$ is competitive (monotone but not cooperative). Finally, the parameters $k_{i}$ are not monotone with respect to the system due to the multi-incidence problem [60], as they appear in several equations with opposite effects.

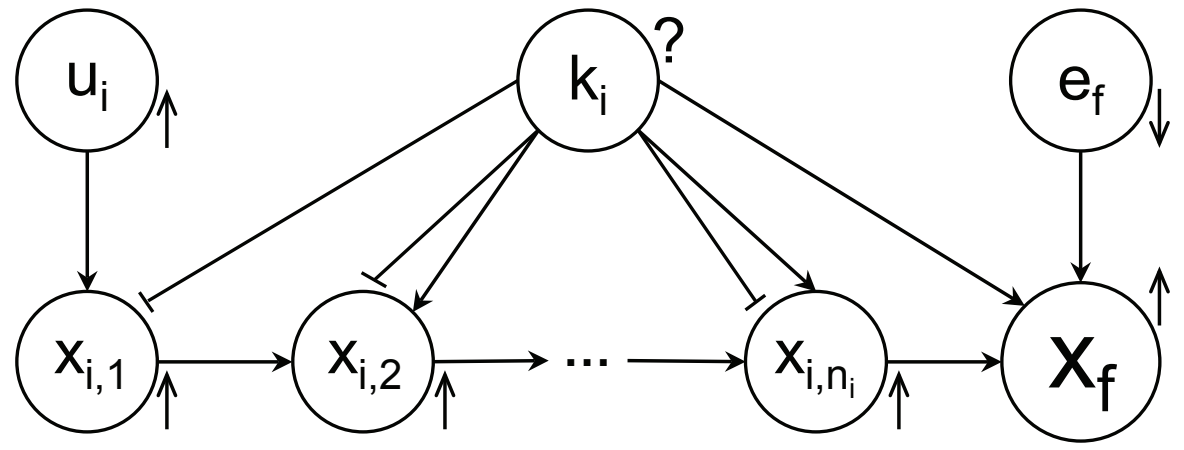

Figure 3.2: Diagram of monotonicity of the parallel inputs model parameters. The parameters $D_{i}$ are cooperative and the parameter $e_{f}$ is competitive, while the parameters $k_{i}$ are not monotone.

When studying the upper (lower) output bound of the parallel inputs model, the maximum (minimum) interval value for the cooperative parameters $D_{i}$, $u_{i}$, and $x_{f, 0}$ is considered, while the minimum (maximum) interval value for the competitive parameter $e_{f}$ is considered. In both cases, the non-monotone parameters $k_{i}$ are still considered as intervals, and they will produce an overestimation on the computation of output bounds. 


\subsection{Proposed method}

After a monotonicity analysis non-monotone compartments and parameters still cause an overestimation in the output bounds computation. Therefore, a new method is proposed from now on based on the use of an equivalent model to the parallel inputs model. As the parallel inputs model is a linear ODE system with the form $Y^{\prime}(t)=A Y(t)$, where $A$ is a square matrix, the solution is given by $Y(t)=e^{A t} Y(0)$ for a constant input. The output solution, $x_{f}(t)$, is not easy to deal with it analytically. However, the solution $x_{i, n_{i}}$ of the last compartment $n_{i}$ of each chain can be studied more easily [8]:

$$
x_{i, n_{i}}(t)=D_{i} e^{-k_{i} t} \frac{\left(k_{i} t\right)^{n_{i}-1}}{\left(n_{i}-1\right) !}+u_{i} \frac{1-e^{-k_{i} t} \sum_{j=1}^{n_{i}-1} \frac{\left(k_{i} t\right)^{j}}{j !}}{k_{i}} .
$$

Analytical solutions of the last compartment of each chain and the ODE associated with the output compartment have been combined, transforming the parallel inputs model into an equivalent and simpler model with just one compartment where inputs are given by (3.2). The new model has thus one input for each chain of the parallel inputs model. The differential equation for the one-compartmental model with $N$ inputs is

$$
\dot{x}_{f}(t)=\sum_{i=1}^{N} k_{i} x_{i, n_{i}}(t)-e_{f} x_{f}(t) \quad x_{f}(0)=x_{f, 0}
$$

where the unique compartment is $x_{f}(t)$, as represented in Figure 3.3 .

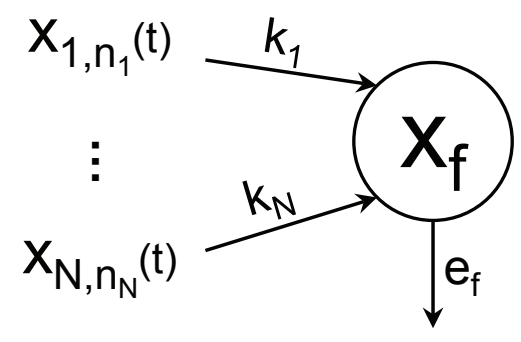

Figure 3.3: Diagram of one-compartment equivalent model.

As the one-compartment model is equivalent to the parallel inputs model, the monotonicity analysis shows that the parameters $D_{i}, u_{i}$, and $x_{f, 0}$ are still cooperative and that the parameter $e_{f}$ is competitive, while the parameters $k_{i}$ are not monotone. The parameters $k_{i}$ are non-monotone in both models, and they produce an overestimation in the output bounds computation. In the parallel inputs model is not possible to analyse these parameters due to their 
multi-incidence. However, in the one-compartment model each $k_{i}$ appears as a parameter for a unique input of the model. Studying the critical points of $\dot{x}_{f}(t)$ with respect to $k_{i}$, there is one non-trivial value:

$$
\frac{\partial \dot{x}_{f}(t)}{\partial k_{i}}=0 \Rightarrow k_{i}=0 \quad \text { or } \quad k_{i}=\frac{n_{i} D_{i}+t u_{i}}{D_{i} t} .
$$

Analysing the second derivative with respect to $k_{i}$, stability of the nontrivial critical point is obtained:

$$
\frac{\partial^{2} \dot{x}_{f}(t)}{\partial k_{i}^{2}}=-\frac{e^{-n_{i}-\frac{u_{i} t}{D_{i}}} t\left(n_{i} D_{i}+u_{i} t\right)^{n_{i}-1}}{\left(n_{i}-1\right) ! D_{i}^{3}} .
$$

As the second derivative is negative, $\dot{x}_{f}(t)$ reaches a maximum at that point. If the critical point (3.4) belongs to the interval $\mathbf{k}_{\mathbf{i}}$ (if uncertainty is considered in its value), it is applied to obtain the upper bound on the solution. Otherwise, the endpoint of the interval $\mathbf{k}_{\mathbf{i}}$ that maximises the output is considered. As there is no minimum, both endpoints of the interval $\mathbf{k}_{\mathbf{i}}$ are analysed, and the value that minimises the output is considered to compute the lower bound.

\subsection{Examples}

Two examples, the double-peak phenomenon and subcutaneous insulin absorption, are studied to verify the effectiveness of the method proposed previously.

The starting point is the result of computing output bounds using the VNODE-LP package [62] for interval analysis. The second computation is performed by using a monotonicity analysis of the parallel inputs model. Finally, the last computation is carried out by evaluating the critical points for the nonmonotone parameters of the one-compartment equivalent model. These last two computations are executed by using Matlab with the toolbox IntLab [76] for interval analysis.

\subsubsection{Double-peak phenomenon}

After the administration of a single dose of several drugs, there is normally a peak in the plasma concentration-time response, before it decays away. However, for certain drugs the plasma concentration rises to a peak, starts to decay, but then it rises again and a second peak is obtained before decaying away again. The second peak is usually higher than the first one, but its magnitude depends on the drug and the means of administration.

Several biological reasons can explain this behaviour, known as the doublepeak phenomenon. The first one is enterohepatic recirculation, which refers to the process in which bile circulates from the liver to the small intestine and 
back to the liver, producing a smaller second peak [68]. The second possible reason affects drugs with high water solubility after oral administration, resulting in part of the dose being delayed in the stomach; this is known as delayed gastric emptying [66]. Finally, the most common reason is variability of absorption within different regions of the guts [54].

The parallel inputs model has been used by Godfrey et al. [30] to model the double-peak phenomenon. Two chains are considered, one for each peak in plasma concentration. One chain is usually smaller and, indeed, faster than the other one, so both peaks are clearly differentiable.

In this example, two chains of ten and five compartments with impulse bolus are considered. The number of compartments of each chain has been arbitrarily chosen to represent an example with longer chains than the subcutaneous insulin absorption example and to demonstrate the method's performance in high-order models. Its differential equations are given by (3.1) with $N=2, n_{1}=10$, and $n_{2}=5$. As the dose is given as an impulse, $u_{1}=u_{2}=0 \mathrm{mU}$, while the initial conditions are $x_{f, 0}=0 \mathrm{mU}, D_{1}=30 \mathrm{mU}$, and $D_{2}=10 \mathrm{mU}$. The absorption rates for each chain are $k_{1}=0.15 \mathrm{~min}^{-1}$ and $k_{2}=0.40 \mathrm{~min}^{-1}$, while the elimination rate is given by $e_{f}=0.20 \mathrm{~min}^{-1}$. A $10 \%$ uncertainty is considered in all the parameters and in the initial conditions of the model.

The VNODE-LP computation, represented in Figure 3.4A, shows that the computed output bounds grow exponentially due to the high number (16) of model compartments, not providing any helpful information. Figure $3.4 \mathrm{~B}$ shows that after performing a monotonicity analysis of the parallel inputs model there is still an overestimation, but the error is much smaller. Finally, a monotonicity analysis of the equivalent one-compartment model is carried out, given by (3.2) and (3.3), where the output bounds adjust almost perfectly to the numerical simulations, as shown in Figure $3.4 \mathrm{C}$.

\subsubsection{Subcutaneous insulin absorption}

Several models of subcutaneous insulin kinetics have been proposed [49, 70], but here this work is focused on one of the most used models, formulated by Wilinska et al. [88]. This subcutaneous insulin absorption model represent two channels of insulin absorption: the slow and the fast channel. Hence, it is composed by two parallel chains $(N=2)$ with different number of compartments $\left(n_{1}=2\right.$ and $n_{2}=1$, respectively), represented by Figure 3.5

The differential equations are given by (3.1), where the absorption rates are $k_{1}=0.0112 \mathrm{~min}^{-1}$ and $k_{2}=0.021 \mathrm{~min}^{-1}$, and the elimination rate is given by $e_{f}=0.0189 \mathrm{~min}^{-1}$. In order to represent insulin concentration in blood, the output result is divided by $V_{i}=0.5645 \mathrm{~L} \mathrm{~kg}^{-1}$ and by the patient weight $B W$.

The proposed method is limited to constant inputs, or bolus inputs as initial conditions. In order to avoid this limitation, the model structure is mod- 

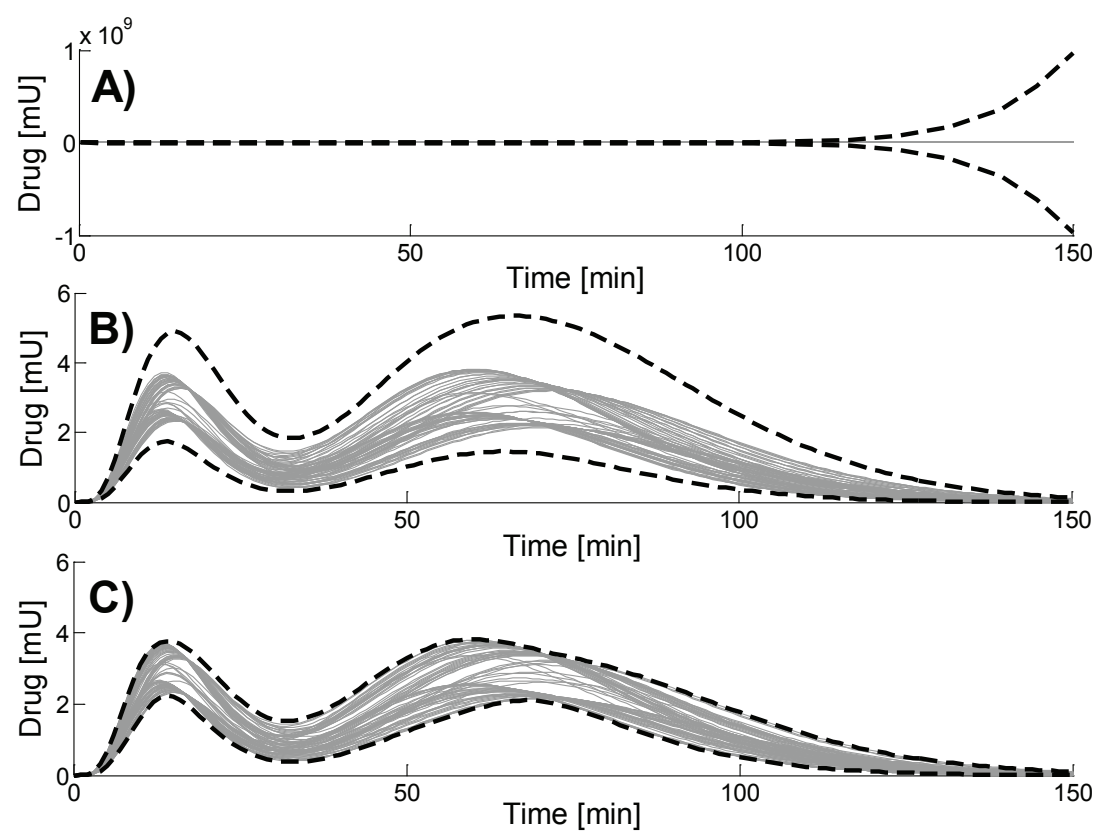

Figure 3.4: Improvements computing double-peak phenomenon bounds. A) VNODE-LP computation. B) Monotonicity analysis of the parallel inputs model. C) Monotonicity analysis of the equivalent one-compartment model.

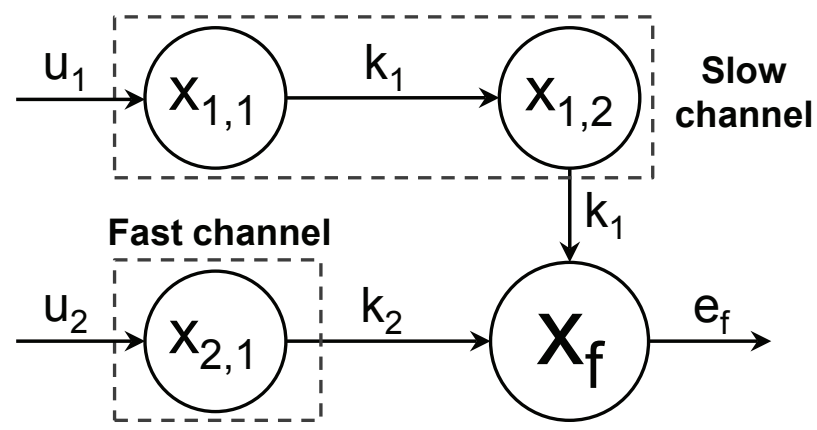

Figure 3.5: Diagram of the parallel inputs model for the Wilinska model.

ified to represent an impulse bolus that occurs at $t=t_{b} \geq 0$. While $t<t_{b}$, the original Wilinska model is considered. However, after the impulse bolus at $t=t_{b}$, two more chains are added to the system with the same absorption rates $k_{1}$ and $k_{2}$ and initial conditions $D_{3}$ and $D_{4}$, which depend on the amount and type of insulin infused. Figure 3.6 shows the new model structure with four chains. 


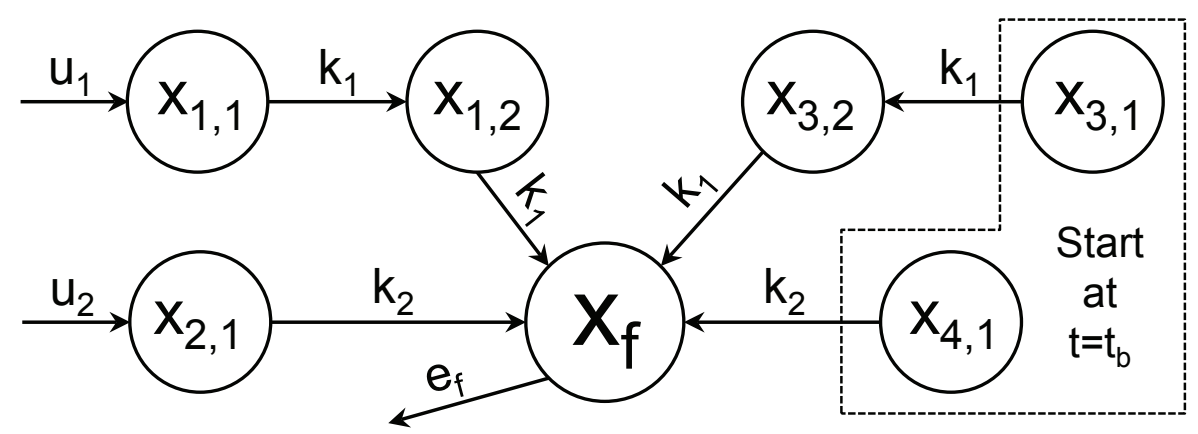

Figure 3.6: Diagram of the parallel inputs model for the extended Wilinska model. Chains 1 and 3 share the parameter $k_{1}$, while chains 2 and 4 share the parameter $k_{2}$.

For the simulation, the insulin bolus considered are $D_{1}=x_{1,1}(0)=445 \mathrm{mU}$, $D_{2}=x_{2,1}(0)=120 m U, D_{3}=x_{3,1}(0)=500 m U$ and $D_{4}=x_{4,1}(0)=250 m U$, with a continuous insulin dose of $u_{1}=5 \mathrm{mU} / \mathrm{min}$ and $u_{2}=2.5 \mathrm{mU} / \mathrm{min}$. Moreover, the output initial condition is given by $x_{f, 0}=395 \mathrm{mU}$, while the patient weight is $B W=70 \mathrm{~kg}$. A $10 \%$ uncertainty is considered in all the parameters and in the initial conditions of the model.

The starting point is given by the VNODE-LP computation in Figure $3.7 \mathrm{~A}$, which shows that the output bounds give a considerable overestimation over the numerical simulations. The output bounds adjust much better after performing a monotonicity analysis of the parallel inputs model, but there is still place for improvements, as shown in Figure 3.73. Finally, the proposed method is evaluated by computing output bounds on the equivalent onecompartment model, given by (3.2) and (3.3). Figure 3.7 C shows that the output bounds adjust almost perfectly to the numerical simulations.

\subsection{Discussion and conclusion}

Different approaches have been applied to compute the set of possible solutions for parallel inputs models when interval parametric uncertainty is considered. In this chapter, a new method is proposed and compared with state-of-the-art approaches in two cases: subcutaneous insulin absorption for artificial pancreas research, and the study of the double-peak phenomenon observed for certain drugs. Each output bounds computation is compared with several possible numerical simulations executed by varying the parameter values inside the intervals. Each numerical simulation takes $0.01 \mathrm{~s}$ to be computed using an Intel(R) $3.2 \mathrm{GHz}$ Pentium(R) processor.

A region-based approach has been considered for the output bounds computation, using VNODE-LP software. This $\mathrm{C}++$ solver computes guaranteed 

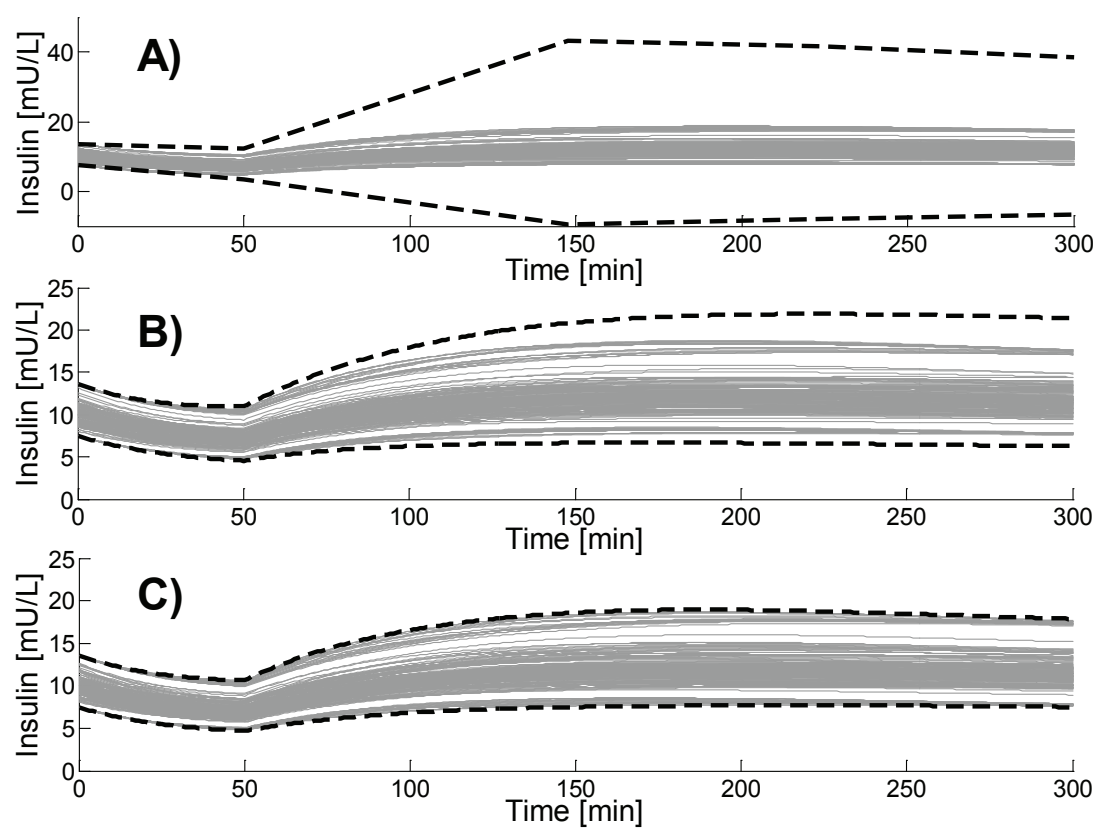

Figure 3.7: Improvements computing subcutaneous insulin absorption bounds with impulse bolus at $t_{b}=50$. A) VNODE-LP computation. B) Monotonicity analysis of the parallel inputs model. C) Monotonicity analysis of the equivalent one-compartment model.

output bounds, but the error seems to increase drastically depending on the number of model compartments: 4 in Figure 3.7A and 16 in Figure 3.4A. A large overestimation is produced in both cases.

A trajectory-based approach has also been considered, performing a monotonicity analysis. Due to the existence of non-monotone parameters $\left(k_{i}\right)$ in the system, an overestimation is produced in the computation of output bounds, as seen in Figure $3.7 \mathrm{~B}$ and Figure $3.4 \mathrm{~B}$. However, this error is much smaller than the one obtained with the VNODE-LP method.

Finally, the proposed method consists in obtaining an equivalent model combining analytical solutions of the input chains with a monotonicity analysis. This approach allows computing critical points of the non-monotone parameter $k_{i}$, which helps to compute tighter output bounds, as seen in Figure 3.7C and Figure 3.4C.

Computing the output bounds with the proposed method takes just 0.02 $\mathrm{s}$, including the computation of the critical point of $k_{i}$ (3.4). Therefore, the proposed method outperforms previous approaches for the computation of output bounds on parallel inputs models, as it minimises the overestimation produced, and also because of its low computational cost.

However, the proposed method has also some limitations, as it can be only 
applied for constant inputs or bolus doses. In order to avoid this limitation, new chains can be considered, as represented in Figure 3.6 The same technique could be applied to any other system with multiple bolus over time.

The approaches shown in this chapter are limited to parallel inputs structures, but the similar ideas can be applied to other systems. Techniques for other type of models will be analysed in the following chapters. 


\section{4 \\ Solution envelopes for in-series model}

Life is a series of natural and spontaneous changes. Don't resist them - that only creates sorrow. Let reality be reality. Let things flow naturally forward in whatever way they like.

Lao-Tsé

\subsection{Introduction}

In-series structures are commonly used in Biology to represent a delay on the output, or to display the relation between physiological compartments. As seen in Section 2.1. this structure is composed of a single chain with several compartments connected in series.

The aim of this chapter is to compute solution envelopes for in-series structures, minimizing the overestimation produced. A novel method is proposed that consists in performing a change of variables in which the output is unaltered, and the model obtained is monotone with respect to the uncertain parameters. The monotonicity of the new system allows us to compute the output bounds for the original system without overestimation.

The method exposed in this chapter has been compared with state-of-theart approaches by the computation of output bounds for two different models: a linear system for glucose modelling and a non-linear system for an epidemiological model.

The work in this chapter has been partially published in the paper Guaranteed computation methods for compartmental in-series models under uncertainty, published by Diego De Pereda, Sergio Romero-Vivo, Beatriz Ricarte and Jorge Bondia in Computers \& Mathematics with Applications 66, 9 (2013), 1595-1605. 


\subsection{In-series models}

A general compartmental in-series model [42], composed of $n$ compartments, is given by the following equations:

$$
\begin{aligned}
& \dot{x}_{1}(t)=u_{1}(t)-\left(k_{1,2}(\cdot)+e_{1}\right) x_{1}(t)+k_{2,1}(\cdot) x_{2}(t) \\
& \dot{x}_{i}(t)=u_{i}(t)+k_{i-1, i}(\cdot) x_{i-1}(t)+k_{i+1, i}(\cdot) x_{i+1}(t)-\left(k_{i, i-1}(\cdot)+k_{i, i+1}(\cdot)+e_{i}\right) x_{i}(t) \\
& \dot{x}_{n}(t)=u_{n}(t)+k_{n-1, n}(\cdot) x_{n-1}(t)-\left(k_{n, n-1}(\cdot)+e_{n}\right) x_{n}(t) \\
& x_{1}(0)=x_{1_{0}}, \quad x_{i}(0)=x_{i_{0}}, \quad x_{n}(0)=x_{n_{0}}
\end{aligned}
$$

for $i \in\{2, \ldots, n-1\}$, where the states of the model $x_{j}(t) \geq 0, j \in\{1, \ldots, n\}$, are the in-series compartments. Furthermore, $u_{j}(t) \geq 0, j \in\{1, \ldots, n\}$, represent the inputs; $e_{j} \geq 0, j \in\{1, \ldots, n\}$, are parameters that denote the elimination rates for each compartment; while $k_{i, j}(\cdot) \geq 0, i, j \in\{1, \ldots, n\}, i \neq j$, are nonnegative scalar functions that represent the flux from the compartment $i$ to the compartment $j$ and they may depend on the states of the model and on a parameter $\alpha_{i, j}$, i.e., $k_{i, j}(\cdot)=k_{i, j}\left(x_{1}(t), \ldots, x_{n}(t), \alpha_{i, j}\right) \geq 0$, such that $\frac{\partial k_{i, j}}{\partial \alpha_{i, j}} \geq 0$. The system will be linear if the fluxes among the compartments are constant, i.e. $k_{i, i+1}(\cdot)=\alpha_{i, i+1}$ and $k_{i+1, i}(\cdot)=\alpha_{i+1, i}, i \in\{1, \ldots, n-1\}$. From now on, $x_{n}(t)$ is considered as the output of the model.

If the fluxes among the compartments of an in-series model go forward and backward, the structure is called bidirectional. However, if the fluxes just go forward, the in-series structure is named unidirectional. Bidirectional inseries structures are shown in Figure 4.1 .

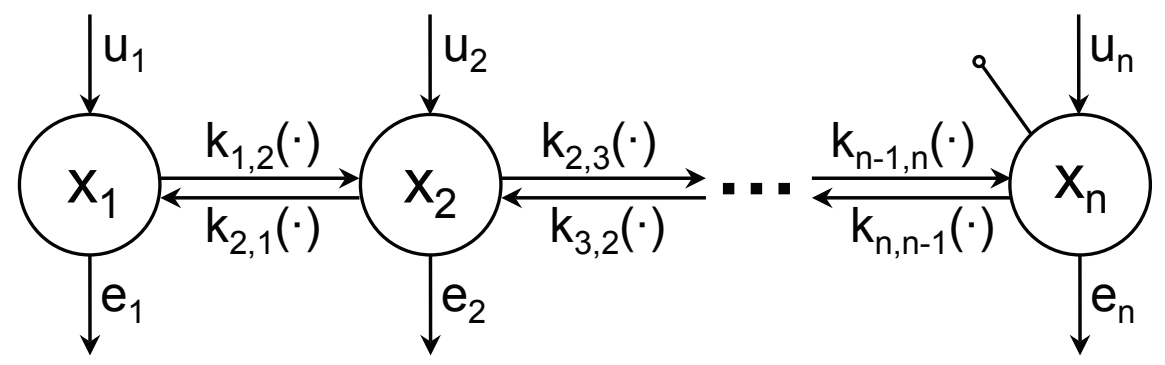

Figure 4.1: Diagram of a compartmental in-series model.

The extended system (including parameters as constant states) is given by adding the following equation to the previous system (4.1):

$$
\dot{p}(t)=0, \quad p(0)=p_{0}
$$

where $p$ encompasses the parameters of the model, i.e., $e_{i}, \alpha_{j, j+1}, \alpha_{j+1, j} \in$ $p, \forall i \in\{1, \ldots, n\}, \forall j \in\{1, \ldots, n-1\}$. 
The monotonicity of a non-linear bidirectional in-series system (4.1) and its extended system given by (4.2) are analysed. Note that it is not possible to determine the exact sign of the partial derivatives $\frac{\partial \dot{x}_{i}(t)}{\partial x_{j}(t)}, i, j \in\{1, \ldots, n\}, i \neq j$. Let us see an example:

$$
\frac{\partial \dot{x}_{1}(t)}{\partial x_{2}(t)}=-\frac{\partial k_{1,2}(\cdot)}{\partial x_{2}(t)} x_{1}(t)+k_{2,1}(\cdot)+\frac{\partial k_{2,1}(\cdot)}{\partial x_{2}(t)} x_{2}(t)
$$

Consequently, some states of the model may be non-monotone. Therefore, the system monotonicity cannot be analysed with respect to the compartments or the parameters of the model through the extension given in (2.2).

\subsection{Proposed method}

The in-series system (4.1) is transformed by performing a change of variables:

$$
S_{i}=\sum_{j=i}^{n} x_{j}(t), \quad \forall i \in\{1, \ldots, n\},
$$

which keeps unaltered the output compartment. This new system is given by:

$$
\begin{aligned}
& \dot{S}_{1}(t)=\sum_{j=1}^{n} u_{j}(t)-\sum_{j=1}^{n-1} e_{j}\left(S_{j}(t)-S_{j+1}(t)\right)-e_{n} S_{n}(t) \\
& \dot{S}_{i}(t)=\sum_{j=i}^{n} u_{j}(t)+k_{i-1, i}(\cdot)\left(S_{i-1}(t)-S_{i}(t)\right)-k_{i, i-1}(\cdot)\left(S_{i}(t)-S_{i+1}(t)\right) \\
& \quad-\sum_{j=i}^{n-1} e_{j}\left(S_{j}(t)-S_{j+1}(t)\right)-e_{n} S_{n}(t) \\
& \dot{S}_{n}(t)=u_{n}(t)+k_{n-1, n}(\cdot)\left(S_{n-1}(t)-S_{n}(t)\right)-\left(k_{n, n-1}(\cdot)+e_{n}\right) S_{n}(t) \\
& \dot{p}(t)=0
\end{aligned}
$$

for $i \in\{2, \ldots, n-1\}$. It is worth mentioning that all the fluxes $k_{i, j}$ in this new system may depend on the new states $S_{i}$, due to the fact that $k_{i, j}(\cdot)=$ $k_{i, j}\left(x_{1}(t), \ldots, x_{n}(t), \alpha_{i, j}\right)$ where $x_{i}(t)=S_{i}(t)-S_{i+1}(t), i \in\{1, \ldots, n-1\}$ and $x_{n}(t)=S_{n}(t)$. Now, for $i \in\{2, \ldots, n-1\}$ :

$$
\begin{cases}\frac{\partial \dot{S}_{1}(t)}{\partial S_{j}(t)}=e_{j-1}-e_{j} & (2 \leq j \leq n), \\ \frac{\partial S_{i}(t)}{\partial S_{j}(t)}=\frac{\partial k_{i-1, i}(\cdot)}{\partial S_{j}(t)}\left(S_{i-1}(t)-S_{i}(t)\right)-\frac{\partial k_{i, i-1}(\cdot)}{\partial S_{j}(t)}\left(S_{i}(t)-S_{i+1}(t)\right) & (j<i-1), \\ \frac{\partial \dot{S}_{i}(t)}{\partial S_{i-1}(t)}=\frac{\partial k_{i-1, i}(\cdot)}{\partial S_{i-1}(t)}\left(S_{i-1}(t)-S_{i}(t)\right)-\frac{\partial k_{i, i-1}(\cdot)}{\partial S_{i-1}(t)}\left(S_{i}(t)-S_{i+1}(t)\right) & \\ \quad+k_{i-1, i}(\cdot), & \\ \frac{\partial \dot{S}_{i}(t)}{\partial S_{i+1}(t)}=\frac{\partial k_{i-1, i}(\cdot)}{\partial S_{i+1}(t)}\left(S_{i-1}(t)-S_{i}(t)\right)-\frac{\partial k_{i, i-1}(\cdot)}{\partial S_{i+1}(t)}\left(S_{i}(t)-S_{i+1}(t)\right) & \\ \quad+k_{i, i-1}(\cdot)+\left(e_{i}-e_{i+1}\right) & \\ \frac{\partial \dot{S}_{i}(t)}{\partial S_{j}(t)}=\frac{\partial k_{i-1, i}(\cdot)}{\partial S_{j}(t)}\left(S_{i-1}(t)-S_{i}(t)\right)-\frac{\partial k_{i, i-1}(\cdot)}{\partial S_{j}(t)}\left(S_{i}(t)-S_{i+1}(t)\right) & \\ \quad+\left(e_{j-1}-e_{j}\right) & (j>i+1), \\ \frac{\partial \dot{S}_{n}(t)}{\partial S_{j}(t)}=\frac{\partial k_{n-1, n}(\cdot)}{\partial S_{j}(t)}\left(S_{n-1}(t)-S_{n}(t)\right)-\frac{\partial k_{n, n-1}(\cdot)}{\partial S_{j}(t)} S_{n}(t) & (j<n-1), \\ \frac{\partial \dot{S}_{n}(t)}{\partial S_{n-1}(t)}=\frac{\partial k_{n-1, n}(\cdot)}{\partial S_{n-1}(t)}\left(S_{n-1}(t)-S_{n}(t)\right)-\frac{\partial k_{n, n-1}(\cdot)}{\partial S_{n-1}(t)} S_{n}(t) & \\ \quad+k_{n-1, n}(\cdot) & \end{cases}
$$

For $i \in\{1, \ldots, n\}$ : 


$$
\begin{aligned}
& \begin{cases}\frac{\partial \dot{S}_{i}(t)}{\partial e_{j}(t)}=0 & \\
\frac{\partial \dot{S}_{i}(t)}{\partial e_{j}(t)}=-\left(S_{j}(t)-S_{j+1}(t)\right) \leq 0 & (i \leq j \leq n-1) \\
\frac{\partial \dot{S}_{i}(t)}{\partial e_{n}(t)}=-S_{n}(t) \leq 0 & \end{cases}
\end{aligned}
$$

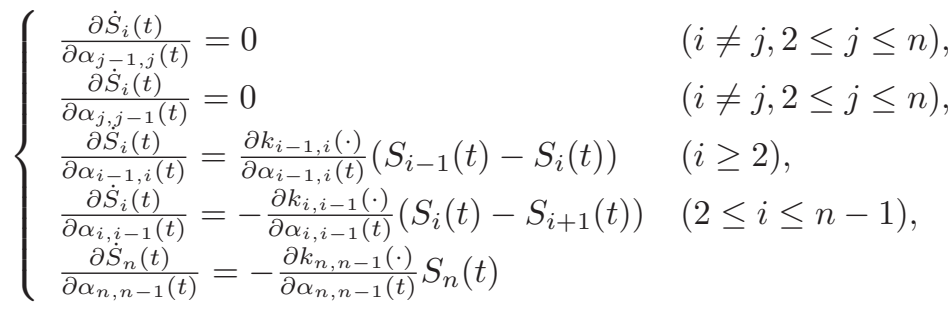

As $\dot{p}=0$, all the partial derivatives of $\dot{p}$ are always null. Now, let us try to ensure the monotonicity of model (4.3). For that, the elimination rates and the fluxes among compartments must satisfy certain conditions. In addition to the relation $e_{j} \geq e_{j+1}, \forall j \in\{1, \ldots, n-1\}$, among the elimination rates, it is also necessary that $\frac{\partial k_{i, i+1}(\cdot)}{\partial S_{j}} \geq 0$ and $\frac{\partial k_{i+1, i}(\cdot)}{\partial S_{j}} \leq 0, \forall i, j: i \in\{1, \ldots, n-1\}, j \in$ $\{1, \ldots, n\}$.

If the above conditions are satisfied, the system is monotone with respect to all the states of the model. The system is cooperative with respect to the states $S_{i}, i \in\{1, \ldots, n\}$, the inputs $u_{j}(t), j \in\{1, \ldots, n\}$, and the parameters $\alpha_{j, j+1}, j \in\{1, \ldots, n-1\}$, while the system is competitive with respect to the elimination rates $e_{j}, j \in\{1, \ldots, n\}$ and the parameters $\alpha_{j+1, j}, j \in\{1, \ldots, n-1\}$.

If the conditions are satisfied, the computation of the bounds for all the new system states is performed without overestimation because the new system is monotone with respect to all the states (states and parameters in the original model). Thus, some specific values of the parameters and the initial conditions are used to compute the upper bound and the lower bound, values that are usually different in each bound. Notice that during the combination of equations, the output compartment remains unchanged because $S_{n}(t)=x_{n}(t)$. Thus, the output of the original system has the same value as the output of the new system. Then, if the same parameters values and initial conditions are applied for the original model, the computation of its output bounds is performed without overestimation. Therefore, the conclusions obtained by the monotonicity analysis of system (4.3) can be applied to original in-series system (4.1). Taking into account the correspondence among the flux derivatives 1 all these relations can be formulated in the following lemma:

$$
\begin{aligned}
& \frac{\partial k(\cdot)}{\partial S_{1}}=\sum_{s=1}^{n} \frac{\partial k(\cdot)}{\partial x_{s}} \frac{\partial x_{s}}{\partial S_{1}}=\frac{\partial k(\cdot)}{\partial x_{1}} \\
& \frac{\partial k(\cdot)}{\partial S_{j}}=\sum_{s=1}^{n} \frac{\partial k(\cdot)}{\partial x_{s}} \frac{\partial x_{s}}{\partial S_{j}}=\frac{\partial k(\cdot)}{\partial x_{j}}-\frac{\partial k(\cdot)}{\partial x_{j-1}} \quad \forall j \in\{2, \ldots, n\}
\end{aligned}
$$

where $k(\cdot)$ represents both $k_{i, i+1}(\cdot)$ and $k_{i+1, i}(\cdot), i \in\{1, \ldots, n-1\}$. 
Lemma 4.3.1. Consider a non-linear in-series model 4.1) characterized by:

(a) The elimination rate for each compartment is greater than or equal to the elimination rate for the next compartment, i.e. $e_{j} \geq e_{j+1}, \forall j \in\{1, \ldots, n-1\}$.

(b) The forward fluxes among the compartments satisfy that $\frac{\partial k_{i, i+1}(\cdot)}{\partial x_{j}}-\frac{\partial k_{i, i+1}(\cdot)}{\partial x_{j-1}} \geq$ 0 , whereas the backward fluxes satisfy that $\frac{\partial k_{i+1, i}(\cdot)}{\partial x_{j}}-\frac{\partial k_{i+1, i}(\cdot)}{\partial x_{j-1}} \leq 0, \forall i, j$ : $i \in\{1, \ldots, n-1\}, j \in\{2, \ldots, n\}$, where $\frac{\partial k_{i, i+1}(\cdot)}{\partial x_{0}}=\frac{\partial k_{i+1, i}(\cdot)}{\partial x_{0}}=0$.

Then, system 4.3), with the same output value as 4.1), satisfies the following properties:

(i) System (4.3) is cooperative with respect to the states $S_{i}, i \in\{1, \ldots, n\}$, the inputs $u_{j}(t), j \in\{1, \ldots, n\}$, and the parameters $\alpha_{j, j+1}, j \in\{1, \ldots, n-1\}$.

(ii) System (4.3) is competitive with respect to the elimination rates $e_{j}, j \in\{1, \ldots, n\}$, and the parameters $\alpha_{j+1, j}, j \in\{1, \ldots, n-1\}$.

Let us set the fluxes among compartments to be constants, i.e. $k_{i, i+1}(\cdot)=$ $\alpha_{i, i+1}$ and $k_{i+1, i}(\cdot)=\alpha_{i+1, i}$. This kind of systems is called linear in-series systems. In this case, the partial derivatives of the fluxes are always equal to zero and the conditions of Lemma 4.3.1 can be simplified.

Corollary 4.3.2. Consider a linear in-series system. If the elimination rate for each compartment is greater than or equal to the elimination rate for the next compartment, i.e. $e_{j} \geq e_{j+1}, \forall j \in\{1, \ldots, n-1\}$, then system (4.3) satisfies the following properties:

(i) System 4.3 is cooperative with respect to the states $S_{i}, i \in\{1, \ldots, n\}$, the inputs $u_{j}(t), j \in\{1, \ldots, n\}$, and the parameters $\alpha_{j, j+1}, j \in\{1, \ldots, n-1\}$.

(ii) System (4.3) is competitive with respect to the elimination rates $e_{j}, j \in\{1, \ldots, n\}$, and the parameters $\alpha_{j+1, j}, j \in\{1, \ldots, n-1\}$.

In the particular case of unidirectional linear in-series systems, where $k_{i, i+1}(\cdot)$ $=\alpha_{i, i+1}$ and $k_{i+1, i}(\cdot)=0, i \in\{1, \ldots, n-1\}$, the conditions can be simplified even more. Consider a single-input single-output (SISO) system [55] with $u_{i}(t)=0, i \in\{2, \ldots, n\}$. Its transfer function is given by:

$$
H(s)=\frac{1}{e_{n}+s} \prod_{i=1}^{n-1} \frac{\alpha_{i, i+1}}{e_{i}+\alpha_{i, i+1}+s}
$$

The transfer function (4.4) will be maintained unaltered [86] if the values of the binomial $\left(\alpha_{i, i+1}, e_{i}\right)$ are exchanged with the values of the binomial $\left(\alpha_{j, j+1}\right.$, $e_{j}$ ) for $i, j \in\{1, \ldots, n-1\}$. In consequence, the output value does not change. Therefore, a compartmental model where $e_{j} \geq e_{n} \forall j \in\{1, \ldots, n-1\}$, can be transformed in a new in-series model, with the same transfer function, where the elimination rates satisfy 


$$
e_{j} \geq e_{j+1}, \forall j \in\{1, \ldots, n-1\} .
$$

This new model satisfies the conditions of Corollary 4.3.2. Therefore, the compartments, the functions $u_{j}(t), j \in\{1, \ldots, n\}$, and the parameters $\alpha_{i, i+1}$ of the new system are cooperative for all $i \in\{1, \ldots, n-1\}$, while the elimination rates $e_{j}, j \in\{1, \ldots, n\}$, are competitive parameters. Hence, the following result is straightforward:

Lemma 4.3.3. Consider a SISO unidirectional linear in-series model with input in the first compartment. If the elimination rate for each compartment is greater than or equal to the elimination rate for the output compartment, i.e. $e_{j} \geq e_{n}, \forall j \in$ $\{1, \ldots, n-1\}$, then system (4.3) satisfies the following properties:

(i) System (4.3) is cooperative with respect to the states $S_{i}, i \in\{1, \ldots, n\}$, the inputs $u_{j}(t), j \in\{1, \ldots, n\}$, and the parameters $\alpha_{j, j+1}, j \in\{1, \ldots, n-1\}$.

(ii) System (4.3) is competitive with respect to the elimination rates $e_{j}, j \in\{1, \ldots, n\}$.

We have just seen that by a change of variables, a new system is obtained with the same output. If Lemma4.3.1 or Lemma4.3.3 conditions are satisfied, the new system is monotone with respect to the states and the parameters of the model. In consequence, the computation of output bounds for this new system is performed without overestimation and hence, the output bounds for the original system. Furthermore, this method produces no limitation in the type of inputs of the model.

When none of the above lemmas is completely satisfied, the computation of output bounds has to be performed directly for the original system. As the non-monotone states and parameters have to be considered as intervals, the output bounds will include a significant overestimation.

However, some systems nearly satisfy the lemma conditions. These kinds of systems are known as near-monotone system [81]. In these cases, based on the theory exposed in [9], a novel method is proposed for the computation of a solution envelope that consists in obtaining an upper bounding model and a lower bounding model of the original model that satisfy the lemma conditions. Then, the computation of the output bounds is performed without overestimation. The area delimited between the lower bound for the lower bounding model and the upper bound for the upper bounding model will guarantee the inclusion of all possible solutions for the original model, and it will minimize the overestimation.

The conditions for Lemma 4.3.1 and Lemma 4.3.3 include special requirements on the elimination rates of the compartmental in-series models. As the elimination rates are competitive parameters, an upper bounding model is obtained at the lower bounds for the elimination rates. Similarly, a lower bounding model is obtained at the upper bounds for the elimination rates. 
For example, Lemma 4.3.3 includes the condition $e_{j} \geq e_{n}, \forall j \in\{1, \ldots, n-$ $1\}$. If the model does not satisfy this requirement, an upper bounding model can be computed such that the elimination rates are given by $\tilde{e}_{j}=e_{j}, j \in$ $\{1, \ldots, n-1\}$, and $\tilde{e}_{n}=\min \left(e_{1}, \ldots, e_{n}\right)$. A lower bounding model is computed by increasing the elimination rate values to $\tilde{e}_{j}=\max \left(e_{j}, e_{n}\right), j \in\{1, \ldots, n-1\}$, and $\tilde{e}_{n}=e_{n}$. In both models the lemma condition $\tilde{e}_{j} \geq \tilde{e}_{n}, \forall j \in\{1, \ldots, n-1\}$ is satisfied.

\subsection{Examples}

In the sequel, the theory presented above is illustrated through two different models. The first example is a linear system for glucose modelling, while the second one is a non-linear system for an epidemiological model. Furthermore, a death rate is added to the second example to analyse a near-monotone model. Namely, a change of variables of the non-monotone systems is performed to obtain new monotone systems, in which output bounds are easily computed without overestimation. Results obtained for both examples are compared with the computation of output bounds following the traditional monotonicity approach [45].

\subsubsection{Linear glucose model}

Cobelli et al. [14, 16] developed a physiological model to describe glucose kinetics. This model is composed by three compartments that describe the nonaccessible portion of insulin system, as seen in Figure 4.2

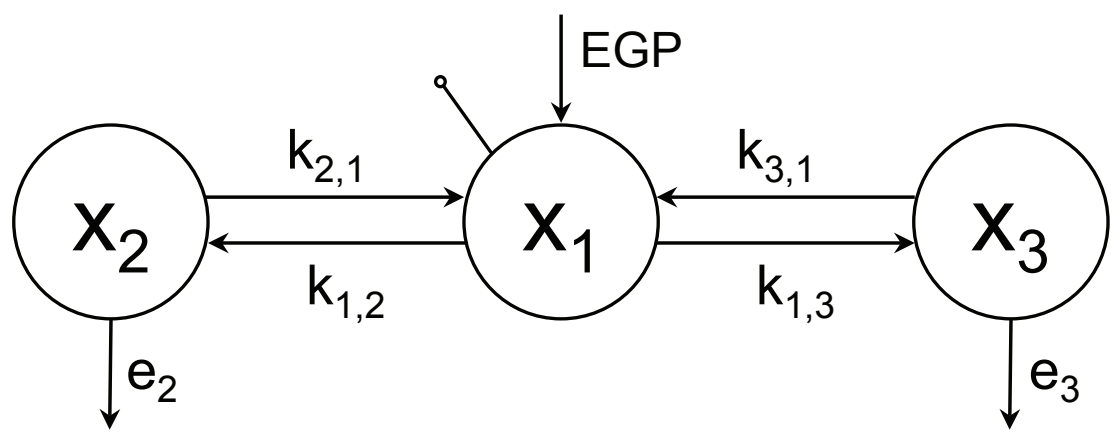

Figure 4.2: Diagram of the linear glucose model developed by Cobelli et al.

The central compartment represents the glucose concentration of the accessible pool. It is the output compartment. The mass balance and measurement equations are given by: 


$$
\begin{aligned}
& \dot{x}_{1}(t)=-\left(k_{1,2}+k_{1,3}\right) x_{1}(t)+k_{2,1} x_{2}(t)+k_{3,1} x_{3}(t)+E G P \\
& \dot{x}_{2}(t)=k_{1,2} x_{1}(t)-\left(k_{2,1}+e_{2}\right) x_{2}(t) \\
& \dot{x}_{3}(t)=k_{1,3} x_{1}(t)-\left(k_{3,1}+e_{3}\right) x_{3}(t) \\
& G(t)=\frac{x_{1}(t)}{V_{I}}
\end{aligned}
$$

where $x_{1}(t)$ is the accessible pool of the plasma glucose, $x_{2}(t)$ and $x_{3}(t)$ illustrate peripheral compartments in rapid and slow equilibrium with the accessible pool, respectively, and the output of the model is given by the plasma glucose concentration $G(t)$, which depends on the central compartment $x_{1}(t)$. The parameter $V_{I}$ is the volume of plasma in the accessible compartment, the parameter EGP denotes the input, the constant parameters $k_{1,2}, k_{1,3}, k_{2,1}$ and $k_{3,1}$ are the fluxes among the compartments, while the parameters $e_{2}$ and $e_{3}$ stand for the elimination rates of the peripheral compartments. In this model there is no elimination rate in the accessible pool.

Performing a monotonicity analysis of the corresponding extended model, it is deduced that system (4.5) is cooperative with respect to the compartments. Furthermore, the input $E G P$ is also a cooperative parameter, while $V_{I}$, and the elimination rates $e_{2}$ and $e_{3}$ are competitive parameters. However, the system is non-monotone with respect to the fluxes $k_{1,2}, k_{1,3}, k_{2,1}$ or $k_{3,1}$, because the partial derivatives of the compartments with respect to the fluxes take different signs. For instance:

$$
\frac{\partial \dot{x}_{1}(t)}{\partial k_{1,2}}=-x_{1}(t)<0 \quad \text { while } \quad \frac{\partial \dot{x}_{2}(t)}{\partial k_{1,2}}=x_{1}(t)>0
$$

However, Cobelli et al. model 4.5 can be analysed as two compartmental in-series models interconnected, where the central compartment is the output of both in-series models. Following the same idea of the change of variables presented in the lemmas, a new monotone model can be found:

$$
\begin{aligned}
& \dot{S}_{1}(t)=-\left(k_{1,2}+k_{1,3}\right) S_{1}(t)+k_{2,1}\left(S_{2}(t)-S_{1}(t)\right)+k_{3,1}\left(S_{3}(t)-S_{1}(t)\right)+E G P \\
& \dot{S}_{2}(t)=-k_{1,3} S_{1}(t)-k_{2,0}\left(S_{2}(t)-S_{1}(t)\right)+k_{3,1}\left(S_{3}(t)-S_{1}(t)\right)+E G P \\
& \dot{S}_{3}(t)=-k_{1,2} S_{1}(t)-k_{3,0}\left(S_{3}(t)-S_{1}(t)\right)+k_{2,1}\left(S_{2}(t)-S_{1}(t)\right)+E G P \\
& \dot{p}(t)=0 \\
& G(t)=\frac{S_{1}(t)}{V_{I}}
\end{aligned}
$$

where $S_{1}=x_{1}, S_{2}=x_{1}+x_{2}, S_{3}=x_{1}+x_{3}$, and all the parameters are included in the parameter vector $p$, i.e., $p=\left[k_{1,2}, k_{1,3}, k_{2,1}, k_{3,1}, e_{2}, k_{3,0}, V_{I}\right]$.

Now, the monotonicity of system (4.6) can be studied: 

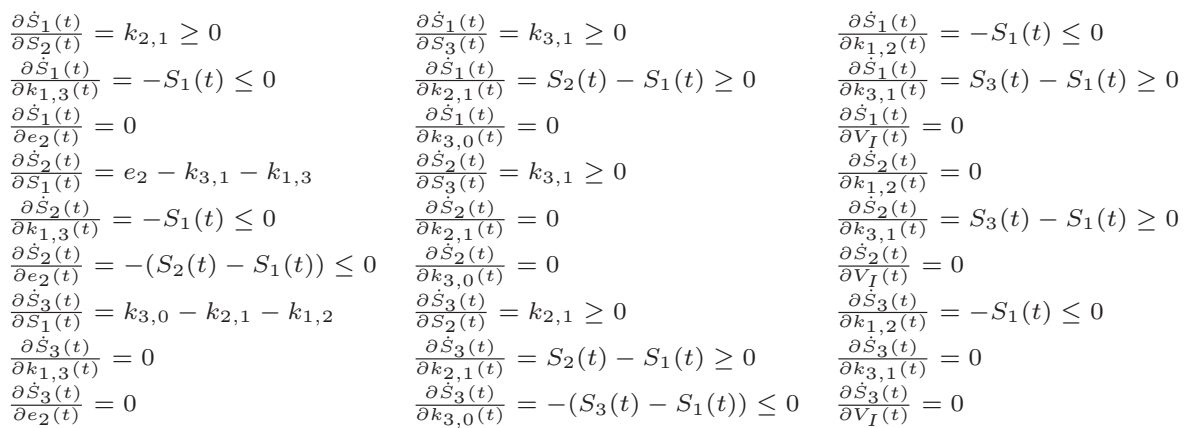

As $\dot{p}=0$, the partial derivatives of $\dot{p}$ are always null. Therefore, system (4.6) is monotone if the conditions $e_{2}-k_{3,1}-k_{1,3}>0$ and $k_{3,0}-k_{2,1}-k_{1,2}>0$ are satisfied. If these conditions are satisfied, the system is cooperative with respect to the states $S_{1}, S_{2}$ and $S_{3}$. Moreover, the input $E G P$, and the parameters $k_{2,1}$ and $k_{3,1}$ are also cooperative, while the parameters $k_{1,2}$ and $k_{1,3}$, the elimination rates $e_{2}$ and $k_{3,0}$, and the volume $V_{I}$ are competitive parameters.

The black dashed lines in Figure 4.3 display the computed output bounds, while the light grey lines represent several numerical simulations executed by the variation of the parameters and initial conditions values. First of all, the computation of output bounds is performed following the traditional monotonicity approach [45], where the non-monotone parameters $k_{1,2}, k_{1,3}, k_{2,1}$ and $k_{3,1}$ produce some overestimation, as seen in Figure 4.3A. Nevertheless, if the monotonicity conditions are satisfied, the system obtained by a change of variables is monotone with respect to all the states and parameters of the model, thus none of them have to be considered as intervals. Therefore, as shown in Figure $4.3 \mathrm{~B}$, the computation of output bounds is performed without overestimation.

\subsubsection{Non-linear epidemiological SIS model}

Epidemiological models have been widely used to assist the decision-making process, which helps to evaluate the consequence of choosing one of the alternate strategies available [3]. Furthermore, epidemic systems have also been used in non-medical areas to study processes that follow an epidemiological behaviour. Here, a SIS model [13] has been analysed, composed by a susceptible population, denoted by $S(t)$, and an infected population, denoted by $I(t)$, as shown in Figure 4.4. Two different cases for the SIS model will be studied depending on whether death by the disease is considered in the system or not. The dynamics of the first model are given by the following equations, in which no disease death rate is considered:

$$
\begin{aligned}
& \dot{S}(t)=\gamma-\beta S(t) I(t)+\alpha I(t)-\nu S(t) \\
& \dot{I}(t)=\beta S(t) I(t)-\alpha I(t)-\nu I(t)
\end{aligned}
$$



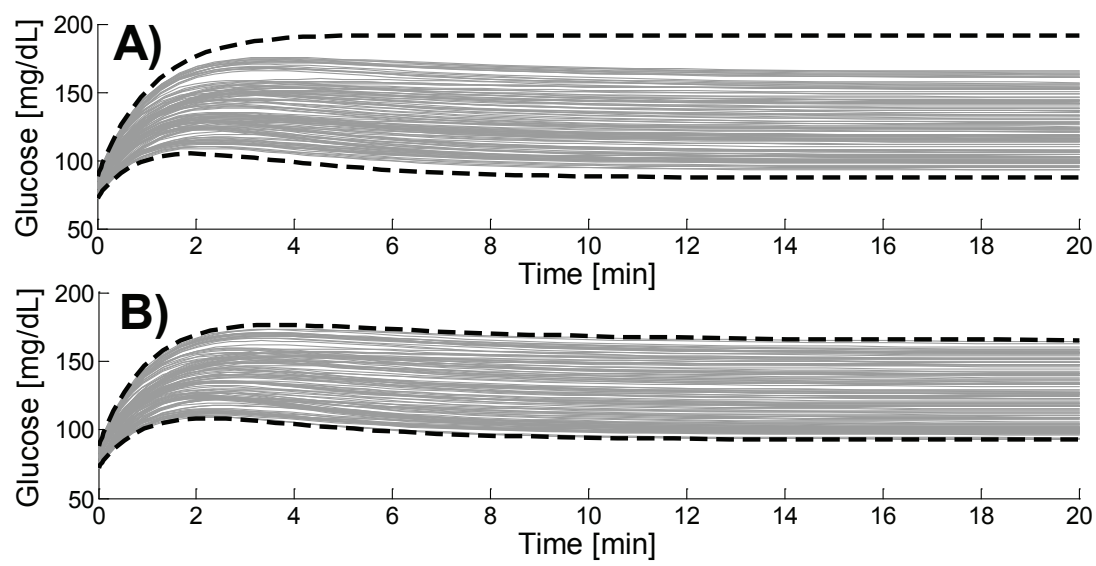

Figure 4.3: Output bounds for the linear glucose model developed by Cobelli et al., where $x_{1}(0)=40, x_{2}(0)=90, x_{3}(0)=90, E G P=22, V_{I}=0.5, e_{2}=0.6$ and $e_{3}=$ 0.55 under $5 \%$ uncertainty, and $k_{1,2}=0.3, k_{2,1}=0.1, k_{1,3}=0.15, k_{3,1}=0.25$ under 15\% uncertainty. A) Monotonicity approach. B) Using system 4.6 when the monotonicity conditions are satisfied.

where the infected population $I(t)$ is the output of the model. The birth rate of the population is represented by the parameter $\gamma$, while the natural death rate is $\nu$. The parameters $\beta$ and $\alpha$ denote the infection rate and the recuperation rate, respectively.

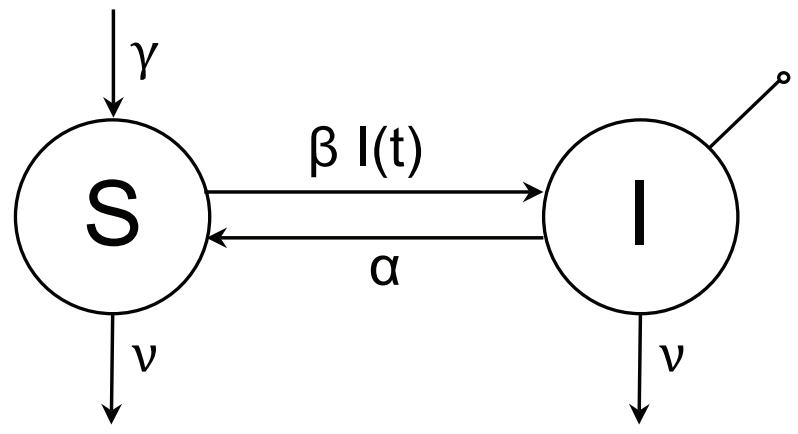

Figure 4.4: Diagram of the non-linear epidemiological SIS model without disease death rate.

Performing a monotonicity analysis of the corresponding extended model, it is deduced that system (4.7) is non-monotone with respect to the states, as the sign of the partial derivative $\frac{\partial \dot{S}(t)}{\partial I(t)}=-\beta S(t)+\alpha$ cannot be determined. Consequently, the system monotonicity with respect to the parameters cannot be evaluated. 
Nevertheless, a change of variables can be performed for system (4.7) to obtain a monotone system with the same output, given by:

$$
\begin{aligned}
& \dot{N}(t)=\gamma-\nu N(t) \\
& \dot{I}(t)=\beta(N(t)-I(t)) I(t)-\alpha I(t)-\nu I(t) \\
& \dot{p}(t)=0
\end{aligned}
$$

where $N(t)=S(t)+I(t)$, and all the parameters are included in the parameter vector $p$, i.e., $p=[\gamma, \beta, \alpha, \nu]$. As the output is unaltered, the conclusions achieved by the computation of the output bounds of 4.8 can also be applied to system (4.7). System (4.8) is cooperative with respect to the compartments, the parameter $\beta$ and the input $\gamma$, while it is competitive with respect to $\alpha$ and the elimination rate $\nu$.

System (4.8), with the same monotonicity properties, can also be obtained applying Lemma 4.3.1 as the lemma conditions are satisfied. The elimination rates of both compartments of the original model are equal to $\nu$. Furthermore, the fluxes between the compartments are given by the function $\beta I(t)$ and the constant parameter $\alpha$, whose partial derivatives satisfy that $\frac{\partial \beta I(t)}{\partial I(t)}-\frac{\partial \beta I(t)}{\partial S(t)}=$ $\beta-0=\beta \geq 0$ and $\frac{\partial \alpha}{\partial I(t)}-\frac{\partial \alpha}{\partial S(t)}=0-0=0 \geq 0$.

Figure 4.5 shows two different computations of the output bounds. The starting point is performed following the traditional monotonicity approach. Figure 4.5A shows that the solution envelope grows exponentially, producing a considerable overestimation over the numerical simulations. Nevertheless, the overestimation disappears when Lemma4.3.1 is applied, as shown in Figure 4.5B. Output bounds are computed without overestimation, as all the states and parameters of the system (4.8) are monotone.
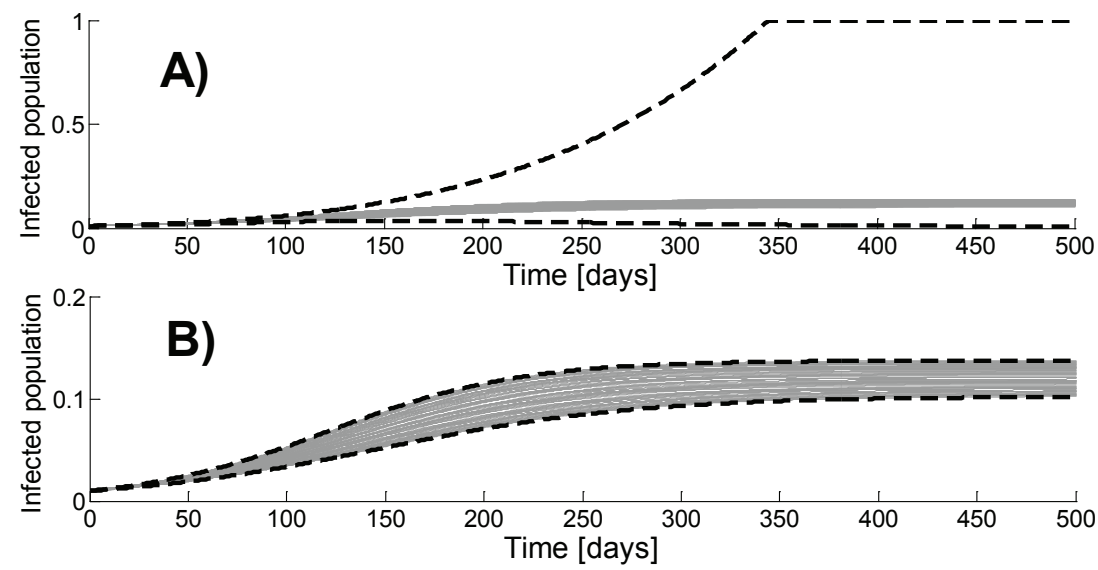

Figure 4.5: Improvements on the computation of bounds for the proportion of infected individuals during a 500 days period, where $I(0)=0.01, S(0)=1-I(0), \gamma=\nu=0.012, \beta=0.15$ and $\alpha=0.12$ under $1 \%$ uncertainty. A) Monotonicity approach. B) Using Lemma 4.3.1 
From now on, a different SIS model will be analysed, in which a death by the disease rate $\theta$ is considered. The dynamic of this model is given by the following equations:

$$
\begin{aligned}
& \dot{S}(t)=\gamma-\beta S(t) I(t)+\alpha I(t)-\nu S(t) \\
& \dot{I}(t)=\beta S(t) I(t)-\alpha I(t)-\nu I(t)-\theta I(t)
\end{aligned}
$$

Unfortunately Lemma 4.3.1 cannot be applied to this system, because the lemma conditions are not completely satisfied, due to the fact that the elimination rates of the compartments $S(t)$ and $I(t)$ are $\nu$ and $\nu+\theta$, respectively. To overcome this trouble, upper and lower bounding models are computed. The upper bounding model is computed by reducing the elimination rate of $I(t)$ to $\nu$, while the lower bounding model is obtained by increasing the elimination rate of $S(t)$ to $\nu+\theta$. Thus, both the upper and the lower bounding models satisfy the Lemma 4.3.1 conditions and guarantee the inclusion of all possible solutions for system 4.9

Again, the solution envelope computed following the traditional monotonicity approach grows exponentially, producing a significant overestimation, as seen in Figure 4.6A. The upper bound for the upper bounding model and the lower bound for the lower bounding model will guarantee the inclusion of all possible solutions of system 4.9 and minimize the overestimation, as seen in Figure $4.6 \mathrm{~B}$.
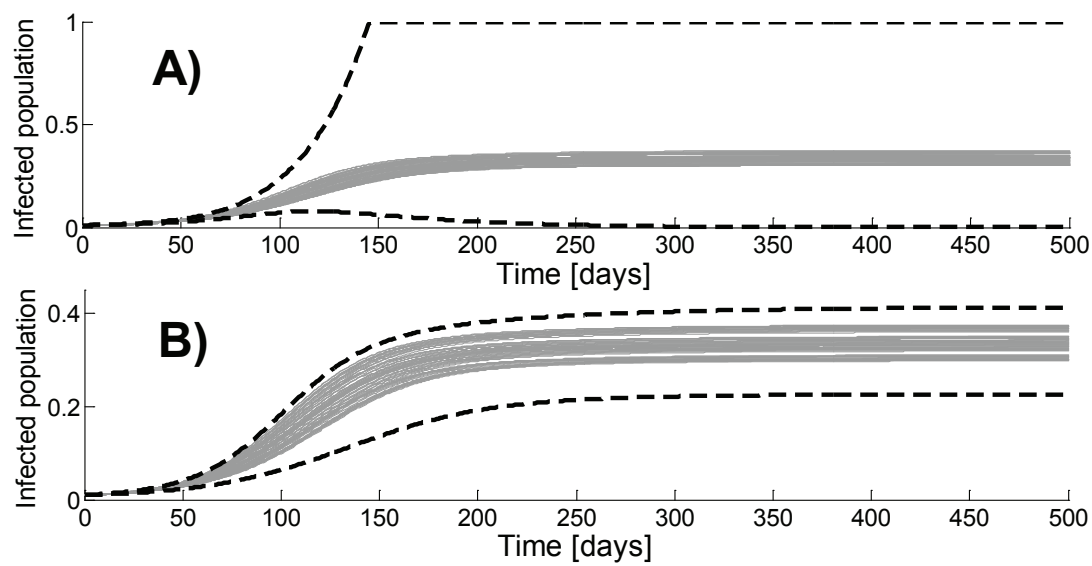

Figure 4.6: Improvements on the computation of bounds for the proportion of infected individuals during a 500 days period considering a death by the disease rate, where $I(0)=0.01$, $S(0)=1-I(0), \gamma=0.015, \nu=0.012, \theta=0.001, \beta=0.15$ and $\alpha=0.12$ under $1 \%$ uncertainty. A) Monotonicity approach. B) Computing an upper bounding model and a lower bounding model, and applying Lemma 4.3.1 on them. 


\subsection{Discussion and conclusion}

The problem of computing tight solution envelopes under parametric uncertainty for models with in-series structures has been tackled in this chapter. A new proposal has been compared with a monotonicity approach in two different examples: a linear system for glucose modelling and a non-linear system for a $S I S$ epidemiological model.

A monotonicity approach of in-series structures exposes the existence of several non-monotone states and parameters. The non-monotonicity of the systems produces an overestimation in the computation of solution envelopes, as seen in Figure 4.3A and Figure 4.5A.

A novel approach is proposed that consists in performing a combination of the equations of the original model to obtain a new model in which the output compartment is unaltered. This new system is monotone with respect to all the compartments and parameters of the model, under assumptions on the elimination rates. As the output of the original model is preserved in the new model, the computed solutions bounds for the new model can be applied to the original model. Therefore, the computation of its output bounds is performed without overestimation, as shown in Figure $4.3 \mathrm{~B}$ and Figure 4.5B. These model transformations have been formulated in Lemma 4.3.1 for bidirectional non-linear chains and in Lemma 4.3.3 for unidirectional linear chains, with more relaxed conditions.

The main limitation of the proposed method is that some necessary lemma conditions have to be satisfied in order to compute the exact solution bounds, with no overestimation at all. However, if the lemma conditions are not completely satisfied it is possible to compute an upper and a lower bounding models that satisfy these conditions. Thus, the solution envelopes for these upper and lower bounding models are computed without overestimation. As the output produced by the model gives smaller values than the upper bounding model and greater than the lower bounding model, both solution envelopes can be combined to compute a tight solution envelope for the original model. This solution envelope guarantees the inclusion of all possible solutions and minimizes the overestimation, as seen in Figure 4.6

Up to now, the preceding chapters were focused on models with some specific structures. However, the following chapter will analyse a method that can be applied to diverse models. 



\section{5}

\section{Solution envelopes based on cone monotonicity}

The only way of discovering the limits of the possible is to venture a little way past them into the impossible.

Arthur C. Clarke

\subsection{Introduction}

In the preceding two chapters, new techniques were developed to compute guaranteed solution envelopes for some specific model structures. The aim of this chapter is to develop a new approach that can be applied to diverse model structures. The most common method in literature to compute output bounds is to perform a monotonicity analysis with respect to the ordering induced by an orthant. However, when the monotonicity conditions are not satisfied, a significant overestimation is derived from numerical simulations. To minimize this overestimation, in this chapter a novel approach is proposed based on monotonicity with respect to an ordering induced by an arbitrary cone, not necessarily an orthant.

This methodology is illustrated with the computation of solution envelopes for a non-linear chemostat model. Two scenarios are considered, with and without the partition of the set of initial state-space conditions.

\subsection{Cone monotonicity analysis}

In this chapter, a more general definition for monotonicity is used, such that the monotonicity analysis is performed with respect to the ordering induced 
by an arbitrary cone [41, 53, 85]. This definition corresponds to the monotonicity approach applied in the preceding chapters when the cone used is the positive orthant. Several basic aspect will be introduced for its application.

Let us recall that a cone $K$ in $\mathbb{R}^{n}$ is a nonempty subset that is closed with respect to the addition and multiplication by nonnegative scalars. According to [6, a cone is called proper if it is closed, it has nonempty interior $(\operatorname{int}(K) \neq$ 0 ), it is a convex set, and it is pointed, i.e. $K \cap-K=\{0\}$ (or equivalently, it contains no lines).

A system is monotone with respect to the ordering induced by a cone $K$ in $\mathbb{R}^{n}$ if for all initial states $x_{1}$ and $x_{2}$, for all parameters $p_{1}$ and $p_{2}$ and for all inputs $u_{1}$ and $u_{2}$ such that $x_{1} \preceq x_{2}, p_{1} \preceq p_{2}$ and $u_{1} \preceq u_{2}$, then

$$
x\left(t ; t_{0}, x_{1}, p_{1}, u_{1}\right) \preceq x\left(t ; t_{0}, x_{2}, p_{2}, u_{2}\right)
$$

for all $t>0$, where $\preceq$ is the relation order induced by the proper cone $K$ in the sense that $x \preceq y$ iff $y-x \in K$ [51, 52, 81].

Under certain assumptions about a smooth map $\gamma: \mathbb{R}^{n} \rightarrow \mathbb{R}$, the differential equation $\dot{x}=f(t, x, u)$ is cooperative with respect to a cone $K$, which is one component of $\left\{x: \gamma(x)>0, x \in \mathbb{R}^{n}\right\}$, if and only if

$$
D \gamma(y) D_{2} f(t, x, u) y \geq 0
$$

for all $y \in \partial K$, where $D \gamma(y)$ is the derivative of $\gamma(y)$, and $D_{2} f(t, x, u)$ denotes the (partial) derivative of $f$ with respect to the second variable $x$ [85].

Example 5.2.1. Define:

$$
\gamma(x)=\left(x_{1}-\frac{x_{2}}{2}\right)\left(\frac{-x_{1}}{2}+x_{2}\right)
$$

and denote by $P$ the component of $\{x: \gamma(x)>0\}$ that is contained in the upper half-plane. Let a system be given by:

$$
\left\{\begin{array}{l}
\dot{x}_{1}(t)=-x_{1}-\frac{x_{2}}{2} \\
\dot{x}_{2}(t)=x_{1}-3 x_{2} .
\end{array}\right.
$$

The partial derivative of the system is given by:

$$
D_{2} f(t, x)=\left(\begin{array}{cc}
-1 & \frac{-1}{2} \\
1 & -3
\end{array}\right)
$$

Then, the system is motonone with respect to the cone $P$ if and only if

$$
D \gamma(y) D_{2} f(t, x) y=\frac{1}{8}\left(18 y_{1}^{2}-44 y_{1} y_{2}+19 y_{2}^{2}\right) \geq 0
$$

Due to the homogeneity of $\gamma$, it is sufficient to check the condition for $y=\left(\frac{1}{2}, 1\right)^{t}$ and $y=\left(1, \frac{1}{2}\right)^{t}$, which yields to 


$$
\begin{aligned}
& D \gamma\left(\frac{1}{2}, 1\right) D_{2} f(t, x)\left(\frac{1}{2}, 1\right)=\frac{3}{16} \geq 0 \\
& D \gamma\left(1, \frac{1}{2}\right) D_{2} f(t, x)\left(1, \frac{1}{2}\right)=\frac{3}{32} \geq 0
\end{aligned}
$$

Therefore, the system is monotone with respect to the cone $P$.

\subsection{Proposed method}

When the system is not monotone with respect to an orthant, a novel approach is proposed to compute tight solution envelopes without increasing the computational cost. This method is based on a monotonicity analysis with respect to the ordering induced by an arbitrary cone [41, 85].

As seen in Figure 2.16 under monotonicity induced by an orthant just two simulations have to be performed to compute the solution bounds, one for the lower bound and another one for the upper bound. However, under monotonicity induced by a cone $K$, a single point of the initial state-space conditions is able to neither lower-bound nor upper-bound all the set of initial state-space conditions (and thus, the trajectories generated from them) as seen in Figure 5.1 .
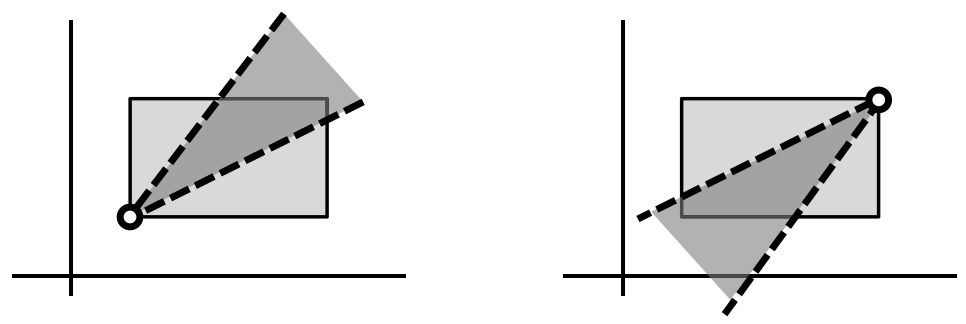

Figure 5.1: Under cone monotonicity, a single endpoint of the set of initial state-space conditions does not cover all the space. Example in $\mathbb{R}^{2}$.

Nevertheless, simulations considering only the boundary of the set of initial state-space conditions do it, as Figure 5.2 represents, and it is proved in the next propositions.

Remark 5.3.1. Likewise as in ordering induced by an orthant, if a cone $K$ contains an orthant and an IVP system 2.1 is monotone with respect to the ordering induced by $K$ then, it is enough to carry out two simulations to compute the solution envelope associated with that system.

Proposition 5.3.2. If a system is monotone with respect to a cone $K$ that contains $l$ semi-axis where $l \in \mathbb{N}, l<n$, then there are two $(n-l)$-dimension polytopes $\bar{R}$ and $\underline{R}$ of $\partial R$ satisfying that

$$
x_{\text {inf }}\left(t ; t_{0}, \underline{R}, p, u\right) \preceq x\left(t ; t_{0}, x_{0}, p, u\right) \preceq x_{\text {sup }}\left(t ; t_{0}, \bar{R}, p, u\right), \quad \forall x_{0} \in R
$$



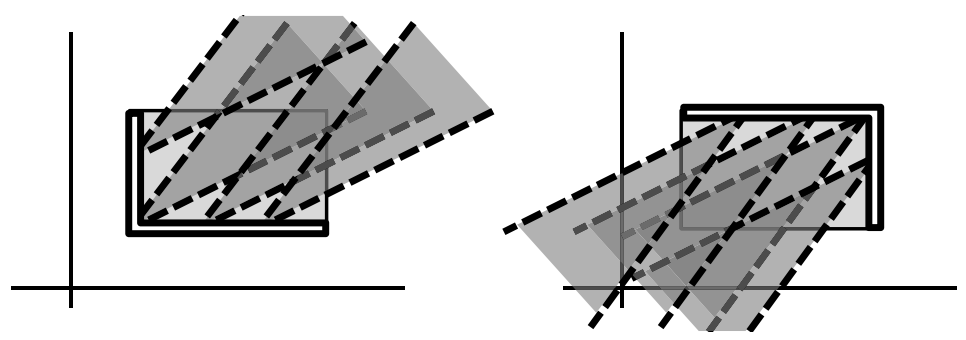

Figure 5.2: Under cone monotonicity, simulations considering the boundary of the set of initial state-space conditions cover all the set of initial state-space conditions. Example in $\mathbb{R}^{2}$.

where

$$
\begin{aligned}
& x_{\text {inf }}\left(t ; t_{0}, \underline{R}, p, u\right)=\inf \left\{x\left(t ; t_{0}, \underline{x}, p, u\right) \mid \underline{x} \in \underline{R}\right\}, \\
& x_{\text {sup }}\left(t ; t_{0}, \bar{R}, p, u\right)=\sup \left\{x\left(t ; t_{0}, \bar{x}, p, u\right) \mid \bar{x} \in \bar{R}\right\}
\end{aligned}
$$

Proof. Knowing that every component $x_{0 i}$ of an initial state $x_{0}$ is included into an interval $\left[x_{0 i}^{-}, x_{0 i}^{+}\right]$, let us suppose that

$$
R=\left[x_{01}^{-}, x_{01}^{+}\right] \times\left[x_{02}^{-}, x_{02}^{+}\right] \times \cdots\left[x_{0 n}^{-}, x_{0 n 1}^{+}\right] \subset \mathbb{R}^{n} .
$$

In addition, for simplicity, let us assume that the $l$ first (positive) semi-axis are included into $K$, that is, the unit vectors $e_{1}, e_{2}, \ldots, e_{l}$ belong to $K$. Let us define the subset providing the necessary initial state-space conditions to simulate the upper bound as follow:

$$
\bar{R}:=\left\{x_{01}^{+}\right\} \times\left\{x_{02}^{+}\right\} \times \cdots\left\{x_{0 l}^{+}\right\} \times\left[x_{0 l+1}^{-}, x_{0 l+1}^{+}\right] \times \cdots \times\left[x_{0 n}^{-}, x_{0 n}^{+}\right] .
$$

Given any $x_{0}=\left(x_{01}, x_{02}, \ldots, x_{0 n}\right) \in R$, the vector

$$
\bar{x}=\left(x_{01}^{+}, x_{02}^{+}, \ldots, x_{0 l}^{+}, x_{0 l+1}, \ldots, x_{0 n}\right)
$$

is chosen, which obviously belongs to $\bar{R} \subset \partial R$ too. Furthermore, since $x_{0 i}^{+}-$ $x_{0 i} \geq 0, \forall i=1, \ldots, n$, $\bar{x}-x_{0}=\left(x_{01}^{+}-x_{01}, \ldots, x_{0 l}^{+}-x_{0 l}, 0, \ldots, 0\right)=\left(x_{01}^{+}-x_{01}\right) e_{1}+\cdots+\left(x_{0 l}^{+}-x_{0 l}\right) e_{l} \in K$ then, $x_{0} \preceq \bar{x}$. Hence, using monotonicity, for any $x_{0} \in R$,

$$
x\left(t ; t_{0}, x_{0}, p, u\right) \preceq x\left(t ; t_{0}, \bar{x}, p, u\right) \preceq x_{\text {sup }}\left(t ; t_{0}, \bar{R}, p, u\right), \quad \forall \bar{x} \in \bar{R} .
$$

In the same way but using $\underline{R}$ where

$$
\underline{R}:=\left\{x_{01}^{-}\right\} \times\left\{x_{02}^{-}\right\} \times \cdots\left\{x_{0 l}^{-}\right\} \times\left[x_{0 l+1}^{-}, x_{0 l+1}^{+}\right] \times \cdots \times\left[x_{0 n}^{-}, x_{0 n}^{+}\right],
$$

it is clear that for any $x_{0} \in R$,

$$
x_{\text {inf }}\left(t ; t_{0}, \underline{R}, p, u\right) \preceq x\left(t ; t_{0}, \underline{x}, p, u\right) \preceq x\left(t ; t_{0}, x_{0}, p, u\right), \quad \forall \bar{x} \in \bar{R} .
$$



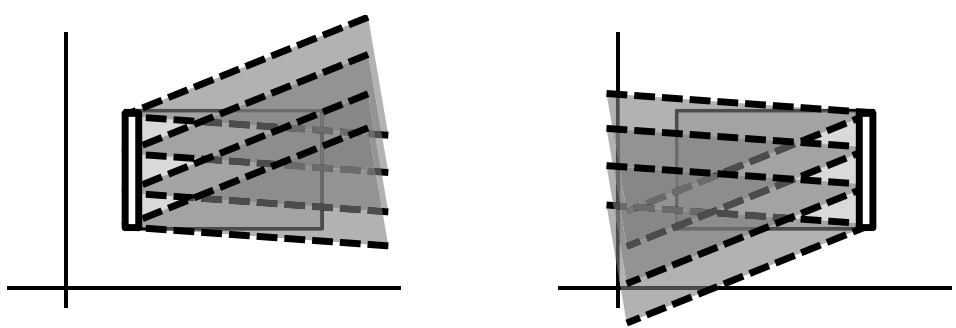

Figure 5.3: Under cone monotonicity, when the cone embraces an axis, that subset $\bar{R}$ ( $\underline{R}$ too) is reduced to a segment (1-dimension polytope) in $\mathbb{R}^{2}$.

Proposition 5.3.2 is graphically illustrated in Figure 5.3.

Let us assume now that $K$ contains neither orthants nor any multiple of a unit vector. Otherwise, reasoning as before, it is obvious that the lower and upper bounds are constructed respectively from only the two polytopes established in the previous proposition. Thus, the cone $K$ can be considered inside an orthant.

Proposition 5.3.3. If a system is monotone with respect to a cone $K$ that it is included into an orthant, then there is a partition of $\partial R$ into two subsets $\bar{R}$ and $\underline{R}$ such that for each $t>0$,

$$
x_{\text {inf }}\left(t ; t_{0}, \underline{R}, p, u\right) \preceq x\left(t ; t_{0}, x_{0}, p, u\right) \preceq x_{\text {sup }}\left(t ; t_{0}, \bar{R}, p, u\right), \forall x_{0} \in R
$$

where

$$
\begin{aligned}
& x_{\text {inf }}\left(t ; t_{0}, \underline{R}, p, u\right)=\inf \left\{x\left(t ; t_{0}, y_{0}, p, u\right) \mid y_{0} \in \underline{R}\right\}, \\
& x_{\text {sup }}\left(t ; t_{0}, \bar{R}, p, u\right)=\sup \left\{x\left(t ; t_{0}, z_{0}, p, u\right) \mid z_{0} \in \bar{R}\right\} .
\end{aligned}
$$

Proof. Let us suppose again that $R=\left[x_{01}^{-}, x_{01}^{+}\right] \times\left[x_{02}^{-}, x_{02}^{+}\right] \times \cdots\left[x_{0 n}^{-}, x_{0 n 1}^{+}\right] \subset \mathbb{R}^{n}$. For the sake of brevity and without losing of generality, let us take the positive orthant as the orthant in which the cone $K$ is included.

Given any $x_{0} \in R$ and any vector $v \in K$ then, the half-line $x_{0}+\lambda v$ intersects $\partial R$ for a large enough $\lambda \geq 0$. By assumptions about $K$, such an intersection point $\overline{x_{0}}$ is located in the subset defined as $\bar{R}=R_{1}^{+} \cup \cdots \cup R_{n}^{+}$ where $R_{i}^{+}=\left[x_{01}^{-}, x_{01}^{+}\right] \times \cdots \times\left\{x_{0 i}^{+}\right\} \times \cdots\left[x_{0 n}^{-}, x_{0 n 1}^{+}\right]$for all $i=1,2, \ldots, n$. Furthermore, $x \preceq \overline{x_{0}}$ since, by the definition of cone, $\overline{x_{0}}-x_{0}=\lambda v \in K$. Thus, using monotonicity, $x\left(t ; t_{0}, x_{0}, p, u\right) \preceq x\left(t ; t_{0}, \overline{x_{0}}, p, u\right), \forall t>0$. Therefore, since this procedure is valid for every initial state-space condition, the upper bound covering all trajectories is derived from simulations computed from the set of initial state-space conditions belonging to $\bar{R}$. More specifically, for each $t>0$,

$$
x\left(t ; t_{0}, x_{0}, p, u\right) \preceq x\left(t ; t_{0}, z_{0}, p, u\right) \preceq x_{\text {sup }}\left(t ; t_{0}, \bar{R}, p, u\right), z_{0} \in \bar{R} .
$$

Similarly but using the cone $-K$ (i.e., $\lambda \leq 0$ ), it can be deduced that the lower bound enveloping all solutions is derived from simulations computed 
from the initial state-space conditions belonging to $\underline{R}:=R_{1}^{-} \cup \cdots \cup R_{n}^{-}$where $R_{i}^{-}=\left[x_{01}^{-}, x_{01}^{+}\right] \times \cdots \times\left\{x_{0 i}^{-}\right\} \times \cdots\left[x_{0 n}^{-}, x_{0 n 1}^{+}\right]$or equivalently, for each $t>0$ :

$$
x_{\text {inf }}\left(t ; t_{0}, \underline{R}, p, u\right) \preceq x\left(t ; t_{0}, y_{0}, p, u\right) \preceq x\left(t ; t_{0}, x_{0}, p, u\right), y_{0} \in \underline{R} .
$$

Finally, notice that $\partial R=\bar{R} \cup \underline{R}$ and $\operatorname{int}(\bar{R}) \cap \operatorname{int}(\underline{R})=\emptyset$, which together with the following order-relation

$$
x_{\text {inf }}\left(t ; t_{0}, \underline{R}, p, u\right) \preceq x\left(t ; t_{0}, x_{0}, p, u\right) \preceq x_{\text {sup }}\left(t ; t_{0}, \bar{R}, p, u\right), \forall x_{0} \in R, \forall t>0,
$$

completes the proof.

Moreover, in order to further reduce the overestimation committed, the boundary of the set of initial state-space conditions $(\partial R)$ can be divided into smaller spaces $\mathcal{R}_{i}$ [60], such that

$$
\partial R=\bigcup_{i} \mathcal{R}_{i} \quad \text { where } \mathcal{R}_{i} \in \mathbb{R}^{n-1} \text { and } \mathcal{R}_{i} \cap \mathcal{R}_{j}=\emptyset, i \neq j
$$

as seen in Figure 5.4
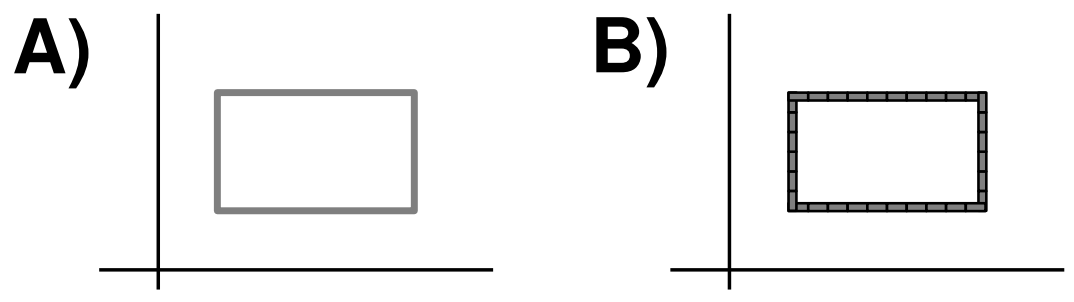

Figure 5.4: A partition of the boundary of the set of initial state-space conditions under cone monotonicity in $\mathbb{R}^{2}$. A) Boundary of the set of initial state-space conditions. B) Division of the boundary of the set of initial state-space conditions.

Similarly to the orthant monotonicity approach (see Figure 2.17), the lower and the upper bounds will be computed with the infimum and the supremum of all the initial state-space conditions simulations:

$$
x_{\text {inf }}\left(t ; t_{0}, \partial R, p, u\right) \preceq x\left(t ; t_{0}, x_{0}, p, u\right) \preceq x_{\text {sup }}\left(t ; t_{0}, \partial R, p, u\right) \quad \forall x_{0} \in R .
$$

where

$$
\begin{aligned}
& x_{\text {inf }}\left(t ; t_{0}, \partial R, p, u\right)=\inf \left\{x_{\text {inf }}\left(t ; t_{0}, \mathcal{R}_{i}, p, u\right), \forall \mathcal{R}_{i}\right\} \\
& x_{\text {sup }}\left(t ; t_{0}, \partial R, p, u\right)=\sup \left\{x_{\text {sup }}\left(t ; t_{0}, \mathcal{R}_{i}, p, u\right), \forall \mathcal{R}_{i}\right\}
\end{aligned}
$$




\subsection{Example: non-linear chemostat model}

The cone monotonicity approach presented in the previous section has been applied to compute guaranteed bounds on the solutions of a non-linear chemostat model. Results obtained have been compared with the computation of solution bounds following the traditional orthant monotonicity approach.

A chemostat is an important laboratory device used in microbial ecology for the continuous culture of microorganisms [80]. It models the competition of several organisms for a single, essential, growth-limiting nutrient supplied at a constant rate [37]. The modeling of a chemostat for a single species of microorganisms is represented by the following equations:

$$
\begin{aligned}
& \dot{x}_{1}(t)=r \cdot\left(k-x_{1}\right)-\beta \cdot x_{1} \cdot x_{2} \\
& \dot{x}_{2}(t)=-r \cdot x_{2}+x_{1} \cdot x_{2}
\end{aligned}
$$

where $x_{1} \geq 0$ denotes the substrate concentration and $x_{2} \geq 0$ the microbes concentration. The parameter $r=5$ stands for the elimination rates, while the parameter $k=5$ represents the input rate. Finally, the parameter $\beta=0.05$ denotes the consumption of the substrate.

Note that there is not orthant monotonicity between the states as the derivatives between the states $x_{1}$ and $x_{2}$

$$
\frac{\partial \dot{x}_{1}(t)}{\partial x_{2}(t)}=-\beta \cdot x_{1} \quad \frac{\partial \dot{x}_{2}(t)}{\partial x_{1}(t)}=x_{2}
$$

have opposite signs. Nevertheless, a general function can be defined

$$
\gamma(x)=\left(a \cdot x_{1}+b \cdot x_{2}\right) \cdot\left(c \cdot x_{1}+d \cdot x_{2}\right)
$$

and a cone $K$ given by $\{x: \gamma(x)>0\}$, and then to analyze the monotonicity of the system with respect to $K$. The partial derivative of 5.1) with respect to the variable $x$ is given by:

$$
D_{2} f(t, x)=\left(\begin{array}{cc}
-r-\beta \cdot x_{2} & -\beta \cdot x_{1} \\
x_{2} & -r+x_{1}
\end{array}\right)
$$

Assuming two points of the boundary of $K$ (for example, $y_{1}=(-b, a)^{t}$ and $\left.y_{2}=(-d, c)^{t}\right)$, the system is monotone with respect to the cone $K$ if the following results are nonnegative:

$$
\left\{\begin{array}{l}
D \gamma\left(y_{1}\right) D_{2} f(t, x) y_{1}=(b \cdot c-a \cdot d) \cdot(b-a \cdot \beta) \cdot\left(-a \cdot x_{1}+b \cdot x_{2}\right) \\
\left.D \gamma\left(y_{2}\right) D_{2} f(t, x) y_{2}=-(b \cdot c-a \cdot d) \cdot(d-c \cdot \beta) \cdot\left(-c \cdot x_{1}+d \cdot x_{2}\right]\right)
\end{array}\right.
$$

for $a, b, c, d \in \mathbb{R}[85]$. Hence, it is possible to select $a=-1, b=0, c=1$ and $d=\beta$. Then,

$$
\left\{\begin{array}{l}
D \gamma\left(y_{1}\right) D_{2} f(t, x) y_{1}=\beta^{2} \cdot x_{1} \\
D \gamma\left(y_{2}\right) D_{2} f(t, x) y_{2}=0
\end{array}\right.
$$


Note that both results are greater than or equal to zero for $x_{1} \geq 0$, thus the monotonicity of the system with respect to cone $K$ is proven. Then, just the boundaries of the initial state-space conditions have to be considered for the computation of guaranteed solution envelopes. In fact, as cone $K$ embraces the vertical axis by Proposition 5.3.2 the solution envelope is obtained by computing just two segments of the initial state-space conditions:

$$
\left\{x_{1} \in\left[x_{1}^{-}, x_{1}^{+}\right], x_{2}=x_{2}^{-}\right\} \cup\left\{x_{1} \in\left[x_{1}^{-}, x_{1}^{+}\right], x_{2}=x_{2}^{+}\right\}
$$

First of all, the computation of solution bounds is performed following the traditional orthant monotonicity approach, in which the system is not monotone. It takes around 0.06 seconds to compute the solution envelope represented with black dotted lines in Figure 5.5, that illustrates the overestimation produced on the substrate and the microbes concentrations. The light grey lines represent several numerical simulations executed for different values of the set of initial state-space conditions. When cone monotonicity approach is applied, the overestimation of the solution bounds computation is reduced in a 74\%, although it remains high, as seen in Figure 5.5 with black dashed lines. The overestimation is computed with respect to the bounds of the Monte Carlo simulations. The computational time is around 0.10 seconds.
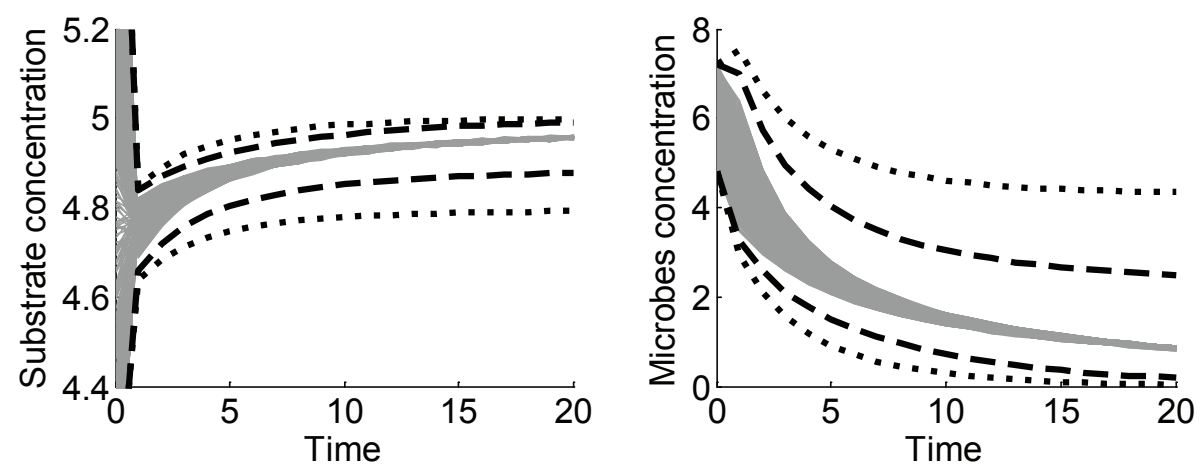

Figure 5.5: Computation of solution envelopes for a non-linear chemostat model with the initial conditions $x_{1}(0)=10$ and $x_{2}(0)=30$ under $20 \%$ uncertainty. Solution envelopes are computed following different approaches: Orthant monotonicity (black dotted line) and cone monotonicity (black dashed line). The grey lines represent several numerical simulations.

Moreover, in order to further reduce the overestimation committed, Figure 5.6 represents the solution envelopes computed when the initial state-space conditions (Figure 2.17) or the boundary of the initial state-space conditions (Figure 5.4) are divided into smaller fragments, respectively. In both cases 36 simulations have been executed, with a computational time of around 1.47 and 1.43 seconds, respectively. The solution envelope computed based on the cone monotonicity approach (black dashed line) is much tighter ( $81 \%$ reduction of the overestimation with respect to the Monte Carlo bounds) than when 
it is computed based on the orthant monotonicity approach (black dotted line), for a similar or even smaller computational cost.
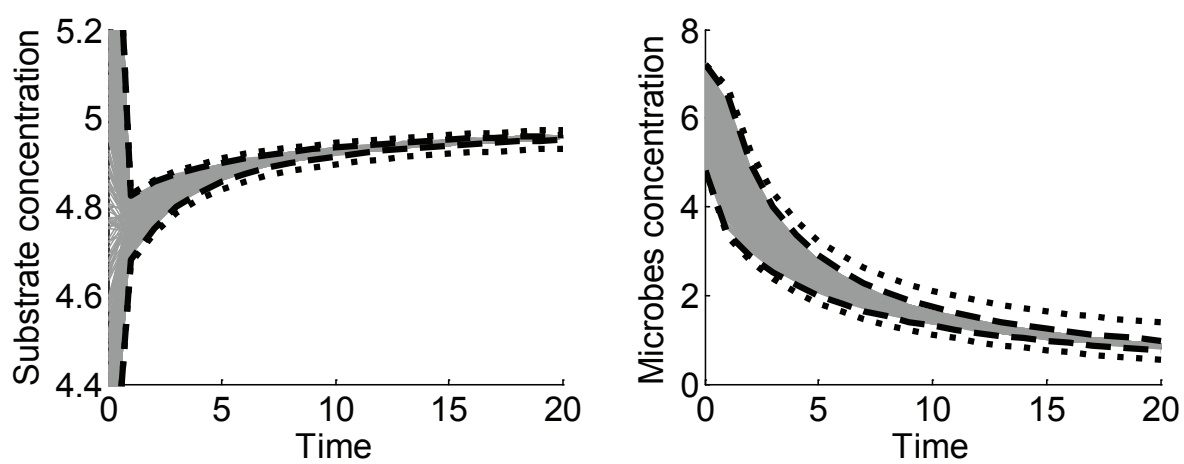

Figure 5.6: Computation of solution envelopes for a non-linear chemostat model with the initial conditions $x_{1}(0)=10$ and $x_{2}(0)=30$ under $20 \%$ uncertainty. The black dotted lines represent the orthant monotonicity approach when there is a partition of the set of initial state-space conditions into 36 segments (according to Figure 2.17). The black dashed lines represent the cone monotonicity approach with a partition of the boundary of the set of initial state-space conditions into 36 segments (in accordance with Figure 5.4.

All the simulations have been executed with Matlab software using an Intel(R) 3.2 GHz Pentium(R) processor.

\subsection{Discussion and conclusion}

In this chapter, a novel approach for the computation of guaranteed solution envelopes has been addressed. This new method has been compared with previous approaches in a non-linear chemostat model.

Traditionally, monotonicity approaches with respect to an orthant have been applied to compute guaranteed solution envelopes under interval uncertainty. Under orthant monotonicity, the exact solution bounds can be computed. However, if there is at least one state without orthant monotonicity, an overestimation will be produced in the solution envelope computation.

For this reason, a novel approach is proposed when the system does not satisfy orthant monotonicity. It is based on performing a monotonicity analysis of the model by taking the ordering induced by an arbitrary cone, not necessarily an orthant. If the system is monotone with respect to a cone $K$ then just the boundary of the set of initial state-space conditions has to be simulated to compute a guaranteed solution envelope, instead of considering all the set of uncertain initial state-space conditions. As the space simulated is reduced, the overestimation committed is also reduced in a $74 \%$, as seen in Figure 5.5 . 
The overestimation can also be reduced by the partition of the set of initial state-space conditions (Figure 2.17) or the boundary of the set of initial condition space (Figure 5.4) into smaller fragments. For a similar computational work, the overestimation of the solution envelopes computed applying the cone monotonicity approach is reduced a $81 \%$ compared with the orthant monotonicity approach, as seen in Figure 5.6 .

In the previous chapters, several changes of variables have been proposed to transform non-monotone systems into monotone systems, which can be computed without overestimation. In those cases, the outputs of the models were unaltered, hence, it was not necessary to undo the changes of variables to compute them. Otherwise, a high overestimation would be produced in this step. Based on a similar idea to the change of variables, a cone approach is proposed in this chapter. For example, a change of variables could be applied in the chemostat model, similar to the cone used. However, doing and undoing the change of variables would create a huge overestimation in the solution bounds computation.

The potential of cone monotonicity analysis is limited, as it is not possible to use it to compute exact output bounds. However, if the system is monotone with respect to any cone, this approach minimises significantly the overestimation produced with respect to the orthant monotonicity analysis for a similar computational cost. 


\section{6 \\ Prediction of glucose concentration under variability}

Marie Curie

\subsection{Introduction}

In the previous chapters, several techniques and approaches have been presented to deal with non-monotone parameters and compute guaranteed solution envelopes, such as analysis of critical points, state transformations or application of differential inequalities. The aim of this chapter is to apply these techniques to compute tight solution bounds for glucose-insulin models to predict the glucose concentration under intra-patient variability in subjects with type 1 diabetes. As a result, it is possible to compute a tight glucose concentration envelope that bounds all the possible patient's glycemic responses.

A preceding contribution by Calm et al. [11] has applied modal interval analysis [27] to compute tight envelopes of glucose concentration under parametric uncertainty. However, only some specific parameters of the glucoseinsulin model were considered uncertain. In this chapter, parametric uncertainty is considered in all the parameters and initial conditions of the model.

The work in this chapter has been partially published in the paper On the prediction of glucose concentration under intra-patient variability in type 1 diabetes: A monotone systems approach, published by Diego De Pereda, Sergio Romero-Vivo, Beatriz Ricarte and Jorge Bondia in Computer Methods and Programs in Biomedicine 108 (2012), 993-1001. 


\subsection{Glucose-insulin model}

The glucose-insulin model is composed of four systems: the carbohydrate digestion and absorption system, the subcutaneous insulin absorption system, the insulin action system, and the glucose metabolism system. Figure 6.1 shows the input-output relationship among these system parts.

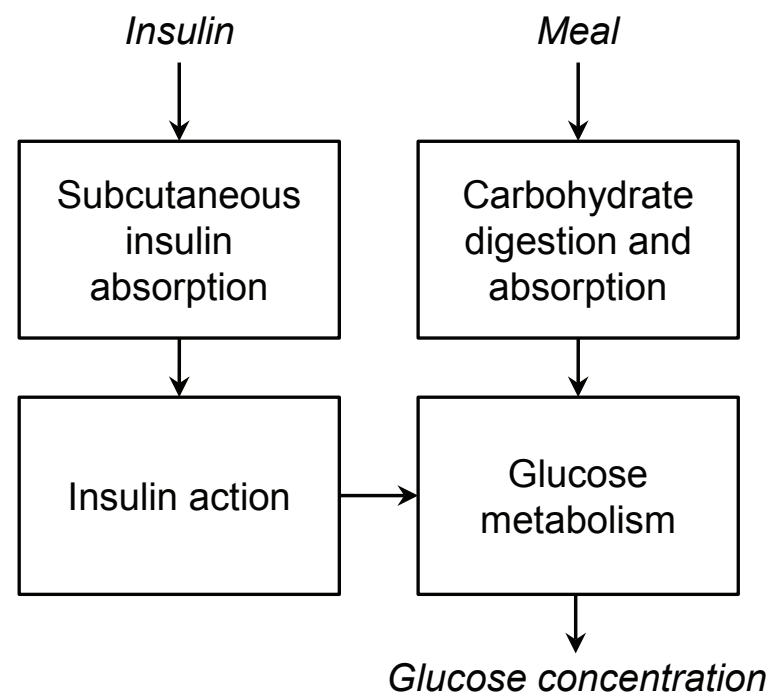

Figure 6.1: Diagram of the systems interaction.

All the systems used in this work are introduced in the glucose-insulin model developed by Hovorka et al. [39], with reported experimental validation results [40]. This is one of the relevant models in literature and it has been chosen to illustrate the technique, although the method could be extended to other models.

\subsubsection{The carbohydrate digestion and absorption system}

This system describes the carbohydrate digestion and its catabolism to glucose. The gut absorption rate $U_{G}(t)(\mathrm{mmol} / \mathrm{min})$ is given by

$$
U_{G}(t)=\frac{D_{G} A_{G} t e^{-t / t_{\max , G}}}{t_{\max , G}^{2}}
$$

where $D_{G}$ (mmol) is the amount of carbohydrates digested, $A_{G}$ (unitless) is the carbohydrate bioavailability, and $t_{\max , G}(\mathrm{~min})$ is the time-of-maximum appearance of glucose in the accessible glucose compartment. 


\subsubsection{The subcutaneous insulin absorption system}

This system calculates how the administered insulin appears in the blood. It is composed by a two-compartment chain with identical transfer rates $1 / t_{\max , I}$ (min) between the two subcutaneous insulin compartments $S_{1}$ and $S_{2}$ :

$$
\begin{aligned}
& \dot{S}_{1}(t)=u(t)-\frac{S_{1}(t)}{t_{\max , I}} \\
& \dot{S}_{2}(t)=\frac{S_{1}(t)}{t_{\max , I}}-\frac{S_{2}(t)}{t_{\max , I}}
\end{aligned}
$$

where $u(t)(\mathrm{mU} / \mathrm{min})$ represents the administration of insulin (basal and bolus), and $t_{\max , I}(\mathrm{~min})$ is the time-to-maximum insulin absorption. The appearance of insulin in the plasma $U_{I}(t)(\mathrm{mU} / \mathrm{min})$ is given by

$$
U_{I}(t)=\frac{S_{2}(t)}{t_{\max , I}}
$$

The plasma insulin concentration $I(t)(\mathrm{mU} / \mathrm{L})$ is represented by

$$
\dot{I}(t)=\frac{U_{I}(t)}{V_{I}}-k_{e} I(t)
$$

where $V_{I}(\mathrm{~L})$ is the insulin distribution volume, and $k_{e}(1 / \mathrm{min})$ is the fractional elimination rate.

\subsubsection{The insulin action system}

The plasma insulin concentration affects the glucose transportation from the plasma to the tissues, the disposal of peripheral glucose, and the production of hepatic glucose. These actions are represented by

$$
\begin{aligned}
& \dot{x}_{1}(t)=-k_{a 1} x_{1}(t)+k_{b 1} I(t) \\
& \dot{x}_{2}(t)=-k_{a 2} x_{2}(t)+k_{b 2} I(t) \\
& \dot{x}_{3}(t)=-k_{a 3} x_{3}(t)+k_{b 3} I(t)
\end{aligned}
$$

where $x_{1}(t)(1 / \mathrm{min})$ represents the effects of insulin on the distribution and transport of glucose, $x_{2}(t)(1 / \mathrm{min})$ stands for the effect on the glucose disposal, while $x_{3}(t)$ (unitless) symbolises the effect on the production of endogenous glucose. The parameters $k_{a i}(1 / \mathrm{min}), i=1,2,3$, are the deactivation rate constants, while $k_{b 1}, k_{b 2}\left(1 / \mathrm{min}^{2}\right.$ per $\left.\mathrm{mU} / \mathrm{L}\right)$ and $k_{b 3}(1 / \mathrm{min}$ per $\mathrm{mU} / \mathrm{L})$ are the activation rate constants.

\subsubsection{The glucose metabolism system}

Finally, the glucose kinetics are represented by a two-compartment system, as follows: 


$$
\begin{aligned}
& \dot{Q}_{1}(t)=-x_{1}(t) Q_{1}(t)+k_{12} Q_{2}(t)-F_{01}^{c}(t)-F_{R}(t)+U_{G}(t) \\
& \quad+E G P_{0}\left(1-x_{3}(t)\right) \\
& \dot{Q}_{2}(t)=x_{1}(t) Q_{1}(t)-\left(k_{12}+x_{2}(t)\right) Q_{2}(t) \\
& G(t)=\frac{Q_{1}(t)}{V_{G}}
\end{aligned}
$$

where $Q_{1}(t)$ and $Q_{2}(t)(\mathrm{mmol})$ are the glucose masses in the accessible compartment and the non-accessible compartment, respectively, while $G(t)(\mathrm{mmol} / \mathrm{L})$ is the glucose concentration in the accessible compartment. The parameter $k_{12}$ $(1 / \mathrm{min})$ is the transfer rate from the non-accessible compartment to the accessible compartment, the parameter $V_{G}(\mathrm{~L})$ denotes the distribution volume of the accessible compartment, and the parameter $E G P_{0}(\mathrm{mmol} / \mathrm{min})$ stands for the endogenous glucose production extrapolated to the zero insulin concentration. The function $F_{01}^{c}(t)(\mathrm{mmol} / \mathrm{min})$ and the parameter $F_{01}$ represent the total non-insulin-dependent glucose disposal, while the function $F_{R}(t)$ is the renal glucose clearance above the glucose threshold of $9 \mathrm{mmol} / \mathrm{L}$ :

$$
\begin{gathered}
F_{01}^{c}(t)=\left\{\begin{array}{cc}
\frac{F_{01} G(t)}{4.5} & \text { if } G(t)<4.5 \mathrm{mmol} / \mathrm{L} \\
F_{01} & \text { if } G(t) \geq 4.5 \mathrm{mmol} / \mathrm{L}
\end{array}\right. \\
F_{R}(t)=\left\{\begin{array}{cc}
0 & \text { if } G(t)<9 \mathrm{mmol} / \mathrm{L} \\
0.003(G(t)-9) V_{G} & \text { if } G(t) \geq 9 \mathrm{mmol} / \mathrm{L}
\end{array}\right.
\end{gathered}
$$

\subsection{Solution envelopes under parametric uncertainty}

In this section, the systems presented in the previous section are analysed by trajectory-based approaches, focusing on the parameters monotonicity. Uncertainty is considered for all the parameters, as well as for all the initial conditions of the states.

\subsubsection{The carbohydrate digestion and absorption system}

The gut absorption rate $U_{G}(t)$ is given by (6.1), in which the parameters $D_{G}$ and $A_{G}$ are cooperative with respect to $U_{G}(t)$, as seen in Figure 6.2. This means that the maximum value of $U_{G}$ is reached only if the maximum values of $D_{G}$ and $A_{G}$ are computed.

On the other hand, $t_{\max , G}$ is a non-monotone parameter, but the critical points of $U_{G}(t)$ with respect to $t_{\max , G}$ can be obtained for a fixed $t$ :

$$
\frac{\partial U_{G}(t)}{\partial t_{\max , G}}=0 \Rightarrow t_{\max , G}=\frac{t}{2}
$$

The sign of the second derivative of $U_{G}(t)$ with respect to $t_{\max , G}$ determines the stability of the critical point $t / 2$ : 


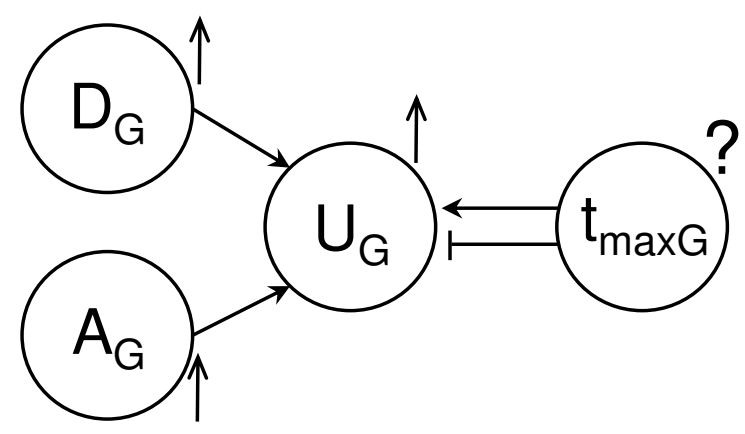

Figure 6.2: Parameters monotonicity of the carbohydrate digestion and absorption system.

$$
\left.\frac{\partial^{2} U_{G}(t)}{\partial t_{\max , G}^{2}}\right|_{t_{\max , G}=t / 2}=-\frac{32 A_{G} D_{G}}{e^{2} t^{3}}
$$

As the second derivative is negative, $U_{G}(t)$ reaches its maximum value at $t_{\max , G}=t / 2$. However, $t / 2$ is not always a possible value for the interval $t_{\max , G}$, depending on the value of $t$ at each time step. If $t / 2$ is not a possible value for the interval $t_{\max , G}$, the maximum value for $U_{G}(t)$ will be attained computing both interval bounds of $t_{\max , G}$ and taking into account the one that maximizes $U_{G}(t)$. On the other hand, the inferior bound of $U_{G}(t)$ is always obtained computing both interval bounds of $t_{\max , G}$ and adopting the one that minimises $U_{G}(t)$.

\subsubsection{The subcutaneous insulin absorption system}

Insulin can be administered in bolus or basal doses, being cooperative with respect to the model in both cases. Bolus are computed as the initial value of the $S_{1}$ compartment (6.2), while $u(t)$ denotes the basal doses (6.2):

$$
S_{1}(0)=u_{\text {bolus }} \quad \text { and } \quad u(t)=u_{\text {basal }}
$$

The problem to solve is a linear ODE system given by (6.2) and (6.3), whose solution [18] is given by

$$
\begin{aligned}
& U_{I}(t)=u_{\text {basal }}+\frac{e^{-\left(t / t_{\max , I}\right)} t\left(-t_{\max , I} u_{\text {basal }}+u_{\text {bolus }}\right)}{t_{\text {max }, I}^{2}} \\
& +e^{-\left(t / t_{\max , I}\right)}\left(-u_{\text {basal }}+U_{I}(0)\right)
\end{aligned}
$$

with two critical points with respect to the parameter $t_{\max , I}$. In order to obtain $U_{I}(t)$ envelope, instead of working with $t_{\max , I}$ as an interval, both critical points (if these values are inside the $t_{\max , I}$ interval) and interval bounds are computed. 
The plasma insulin concentration $I(t)$, given by (6.4), is cooperative with respect to $U_{I}(t)$, while the parameters $V_{I}$ and $k_{e}$ are competitive, as seen in Figure 6.3.

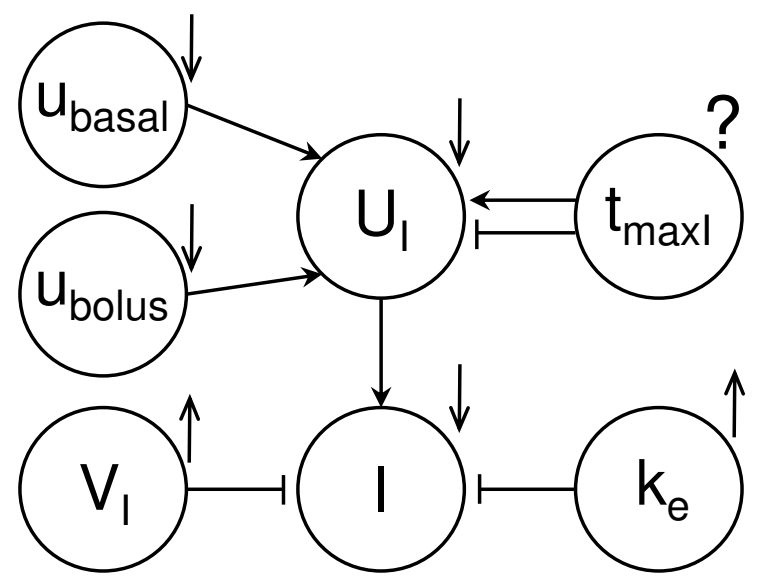

Figure 6.3: Parameters monotonicity of the subcutaneous insulin absorption system.

\subsubsection{The insulin action system}

The effects of insulin on the glucose transport from the plasma to the tissues, the disposal of the peripheral glucose, and the production of hepatic glucose are given by (6.5). Analysing the equations of the system, it is clear that $I(t)$ and $x_{i}, i=1,2,3$ are all cooperative. Furthermore, the parameters $k_{b i}, i=1,2,3$, are also cooperative, while the parameters $k_{a i}, i=1,2,3$, are competitive, as seen in Figure 6.4.

\subsubsection{The glucose metabolism system}

The equations of the glucose metabolism system (6.6) are modified to avoid the parameters multi-incidence in different equations. First of all, the noninsulin-dependent glucose disposal (6.7) and the renal glucose clearance (6.8) are transformed such that $F_{01}^{c}(t)+F_{R}(t)=F C(t) G(t)+F R(t)$, as done in [11], where

$$
F C(t)=\left\{\begin{array}{cl}
\frac{F_{01}}{4.5} & \text { if } G(t)<4.5 \mathrm{mmol} / \mathrm{L} \\
0.003 \cdot V_{G} & \text { if } G(t) \geq 9 \mathrm{mmol} / \mathrm{L} \\
0 & \text { otherwise }
\end{array}\right.
$$




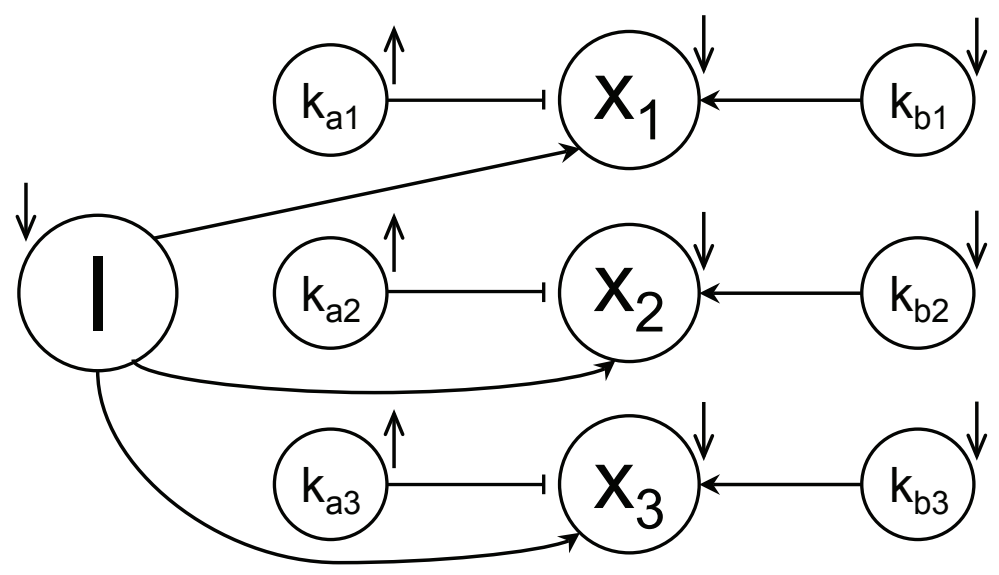

Figure 6.4: Parameters monotonicity of the insulin action system.

$$
F R(t)=\left\{\begin{array}{cl}
0 & \text { if } G(t)<4.5 \mathrm{mmol} / \mathrm{L} \\
F_{01}-0.027 \cdot V_{G} & \text { if } G(t) \geq 9 \mathrm{mmol} / \mathrm{L} \\
F_{01} & \text { otherwise }
\end{array}\right.
$$

As $G(t)$ is given by $G(t)=Q_{1}(t) / V_{G}$, then $\dot{G}(t)=\dot{Q}_{1}(t) / V_{G}$. Thus, by expression (6.6):

$$
\begin{aligned}
& \dot{G}(t)=-x_{1}(t) G(t) \\
& \quad+\frac{k_{12} Q_{2}(t)-F C(t) G(t)-F R(t)+U_{G}(t)+E G P_{0}\left(1-x_{3}(t)\right)}{V_{G}} \\
& \dot{Q}_{2}(t)=x_{1}(t) V_{G} G(t)-\left(k_{12}+x_{2}(t)\right) Q_{2}(t)
\end{aligned}
$$

To avoid the parameter $V_{G}$ multi-incidence in both equations, a new state $H(t)=Q_{2}(t) / V_{G}$ is defined. Hence:

$$
\begin{aligned}
\dot{G}(t) & =-x_{1}(t) G(t)+k_{12} H(t) \\
+ & \frac{-F C(t) G(t)-F R(t)+U_{G}(t)+E G P_{0}\left(1-x_{3}(t)\right)}{V_{G}} \\
\dot{H}(t) & =x_{1}(t) G(t)-\left(k_{12}+x_{2}(t)\right) H(t)
\end{aligned}
$$

Finally, to avoid the multi-incidence of the state $x_{1}(t)$ and the parameter $k_{12}$, another new state $S(t)=H(t)+G(t)$ is computed:

$$
\begin{aligned}
\dot{G}(t) & =-x_{1}(t) G(t)+k_{12}(S(t)-G(t)) \\
+ & \frac{-F C(t) G(t)-F R(t)+U_{G}(t)+E G P_{0}\left(1-x_{3}(t)\right)}{V_{G}} \\
\dot{S}(t) & =-x_{2}(t)(S(t)-G(t)) \\
+ & \frac{-F C(t) G(t)-F R(t)+U_{G}(t)+E G P_{0}\left(1-x_{3}(t)\right)}{V_{G}}
\end{aligned}
$$


The condition $\operatorname{sign}(\partial \dot{G}(t) / \partial S(t))=\operatorname{sign}(\partial \dot{S}(t) / \partial G(t))$ is necessary to prove the monotonicity between the states $G(t)$ and $S(t)$. As $\partial \dot{G}(t) / \partial S(t)=k_{12} \geq 0$, both states are cooperative if $\partial \dot{S}(t) / \partial G(t)=x_{2}(t)-F C(t) / V_{G}$ is non-negative.

As this condition is not always true, two models are computed [19, 21]: an upper bounding model and a lower bounding model, in which elimination rates are modified to satisfy $x_{2}(t)-F C(t) / V_{G} \geq 0$. In the lower bounding model, the state $x_{2}(t)$ is replaced by $\max \left(x_{2}(t), F C(t) / V_{G}\right)$, increasing its value. As $x_{2}(t)$ is a competitive state, the model obtained is a lower bounding model [9] of the model (6.9), and such that

$$
\begin{aligned}
& \dot{G}_{1}(t)=-x_{1}(t) G_{1}(t)+k_{12}\left(S_{1}(t)-G_{1}(t)\right) \\
& +\frac{-F C(t) G_{1}(t)-F R(t)+U_{G}(t)+E G P_{0}\left(1-x_{3}(t)\right)}{V_{G}} \\
& \dot{S}_{1}(t)=-\max \left(x_{2}(t), \frac{F C(t)}{V_{G}}\right)\left(S_{1}(t)-G_{1}(t)\right) \\
& \quad+\frac{-F C(t) G_{1}(t)-F R(t)+U_{G}(t)+E G P_{0}\left(1-x_{3}(t)\right)}{V_{G}}
\end{aligned}
$$

For the upper bounding model, $F C(t) / V_{G}$ is replaced by $\min \left(x_{2}(t), F C(t) / V_{G}\right)$, decreasing its value. As $F C(t) / V_{G}$ is competitive, an upper bounding model of (6.9) is obtained, such that

$$
\begin{gathered}
\dot{G}_{2}(t)=-x_{1}(t) G_{2}(t)+k_{12}\left(S_{2}(t)-G_{2}(t)\right)-\min \left(x_{2}(t), \frac{F C(t)}{V_{G}}\right) G_{2}(t) \\
+\frac{-F R(t)+U_{G}(t)+E G P_{0}\left(1-x_{3}(t)\right)}{V_{G}} \\
\dot{S}_{2}(t)=-x_{2}(t)\left(S_{2}(t)-G_{2}(t)\right)-\min \left(x_{2}(t), \frac{F C(t)}{V_{G}}\right) G_{2}(t) \\
\quad+\frac{-F R(t)+U_{G}(t)+E G P_{0}\left(1-x_{3}(t)\right)}{V_{G}}
\end{gathered}
$$

where $6.10 \leq 6.96$ 6.11) and $G_{i}(t)$ and $S_{i}(t), i=1,2$, are cooperative systems. The system (6.10) is used to calculate the lower bound of $G(t)$, and the system (6.11) for the upper bound. In both systems, the states $x_{i}(t), i=1,2,3$, are competitive, while the state $U_{G}(t)$ is cooperative. Furthermore, the parameter $k_{12}$ is cooperative and $F_{01}$ is competitive. Finally, the parameter $E G P_{0}$ is monotone for all instant $t$, but it can be cooperative if $\left(1-x_{3}(t)\right)>0$, or competitive otherwise. Also, the parameter $V_{G}$ is always monotone for all instant $t$, but can be cooperative or not depending on whether it divides a positive or a negative quantity, respectively. Thus both intervals bounds for the parameters $E G P_{0}$ and $V_{G}$ have to be computed depending on the condition, as seen in Figure 6.5 


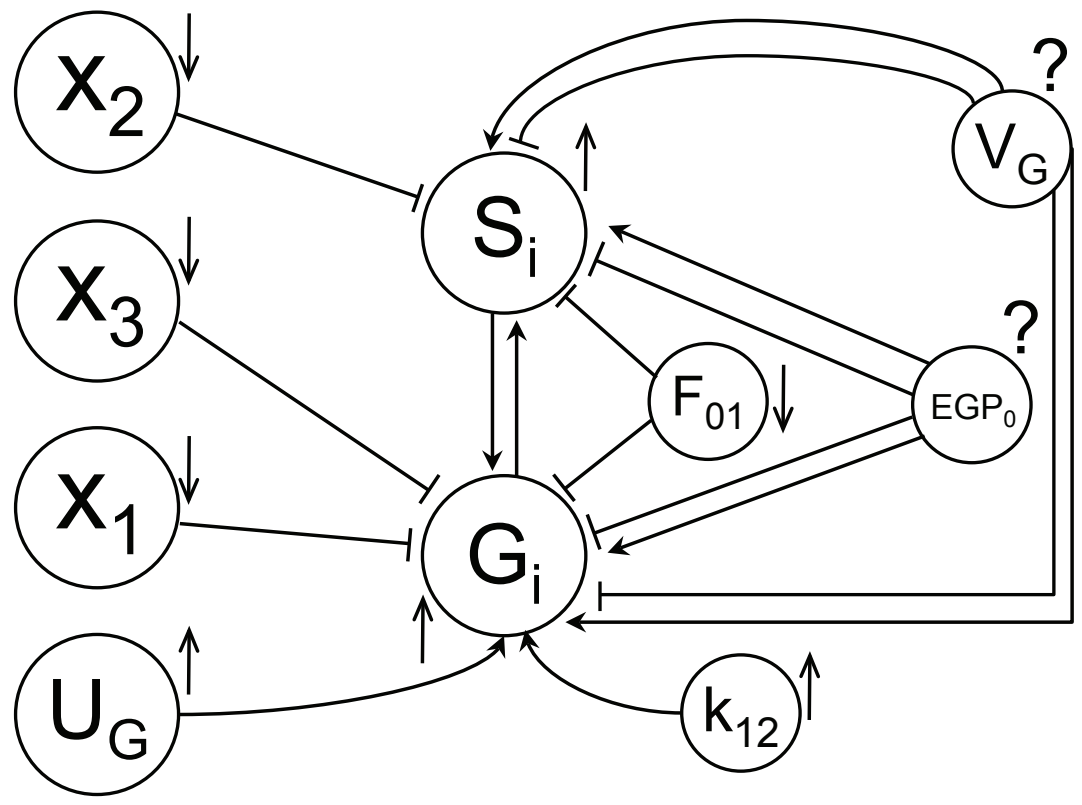

Figure 6.5: Parameters monotonicity of the glucose metabolism system.

\subsection{Results}

The glucose-insulin model developed by Hovorka et al. [39] has been analysed under parametric uncertainty. The parameter values used in all the systems of the model have been taken from [39]. As an illustration of the overestimation made, $5 \%$ uncertainty has been considered in all the parameters and initial conditions of the model. The simulations have been performed for a patient weight of $80 \mathrm{~kg}$, during the 5 hour period that follows a meal of $60 \mathrm{~g}$ and an insulin dose of $3 \mathrm{U}$, taking into account an insulin basal infusion of $0.32 \mathrm{U} / \mathrm{h}$.

All the simulations have been executed with Matlab software (version R2007b) using an Intel(R) 3.2 GHz Pentium(R) processor. Solution envelopes have been computed for two types of situations: long-term predictions during a 5-hour postprandial period, and short-term predictions in which the glucose concentration is measured every 15 minutes.

\subsubsection{Long-term glucose prediction}

In the long-term prediction examples a unique glucose measurement is performed, at time zero. Different scenarios have been computed by varying the initial blood glucose in each simulation. The resulting solution envelope has been compared with numerical simulations to estimate the overestimation produced. The light grey lines represent several Monte Carlo simulations 
performed by varying the parameters and initial conditions values, while the black dashed lines represent the computed output bounds.

In the first simulation, a safe initial condition of $150 \mathrm{mg} / \mathrm{dL}$ was computed, as seen in Figure6.6 In the second scenario, the initial condition was near hypoglycemia with $80 \mathrm{mg} / \mathrm{dL}$, as seen in Figure 6.7 Finally, an initial condition near hyperglycemia with $250 \mathrm{mg} / \mathrm{dL}$ was computed, as seen in Figure 6.8 .

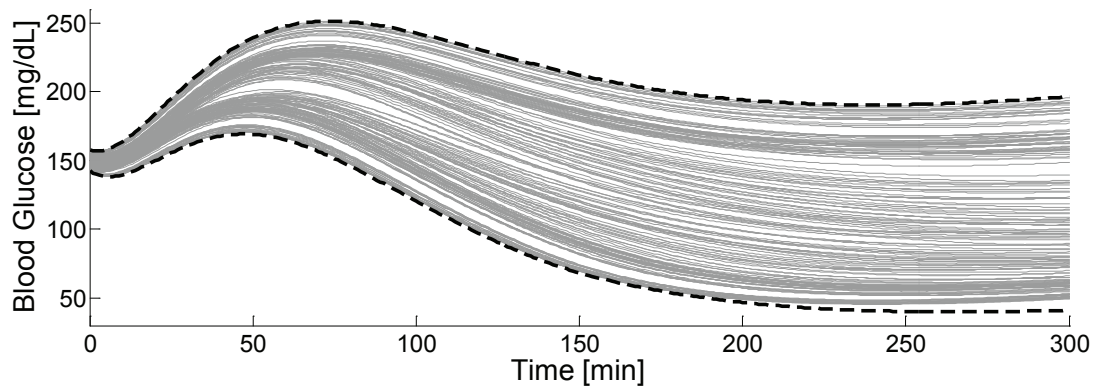

Figure 6.6: Solution envelope obtained for the first scenario.

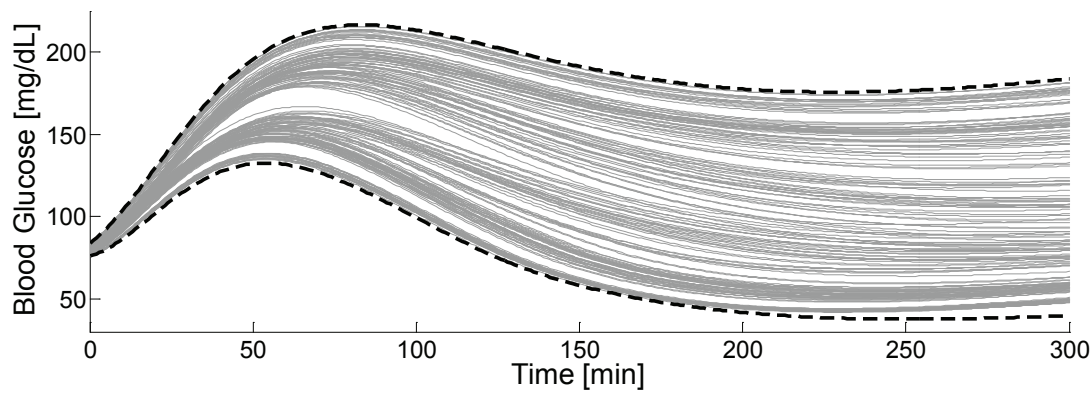

Figure 6.7: Solution envelope obtained for the second scenario.

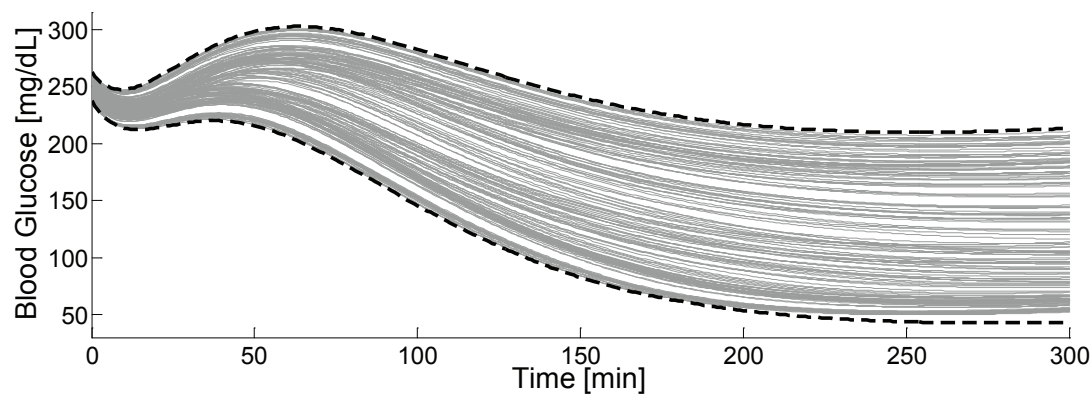

Figure 6.8: Solution envelope obtained for the third scenario. 
In the case of long-term predictions, the computed envelope is almost perfect during the first 200 minutes, and then only a small overestimation is made in the lower bound. The total error is smaller than $1.85 \%$, and it is measured by comparing the area of the numerical simulations with the area of the computed solution envelope. The computational cost does not depend on the number of uncertain parameters, and it takes around 0.027 seconds to obtain the solution envelope, using an explicit Runge-Kutta method for the numerical simulation of the bounding systems. It is noteworthy that the consideration of only $5 \%$ uncertainty in all the parameters translates into a wide range of possible patient responses from hypoglycemia to hyperglycemia. Note that this is not due to the simulation overestimation, since the same effect is observed in the Monte Carlo simulations. This fact illustrates the difficulty of glycemic control in clinical practice.

\subsubsection{Short-term glucose prediction}

In the previous example, the glucose level is only measured initially. However, continuous glucose monitoring allows for the frequent measurement of the glucose level in sensor-augmented pumps and the artificial pancreas. In this case, short-term predictions are used at each sample period to predict hypoglycemia or decide the optimal insulin infusion. In this short-term simulation, 30-minutes-ahead predictions are performed every 15 minutes, after each glucose level measurement (generated from a random nominal value for the parameters inside the given intervals). As glucose measurements may include an error, $5 \%$ uncertainty is considered in all the glucose values.

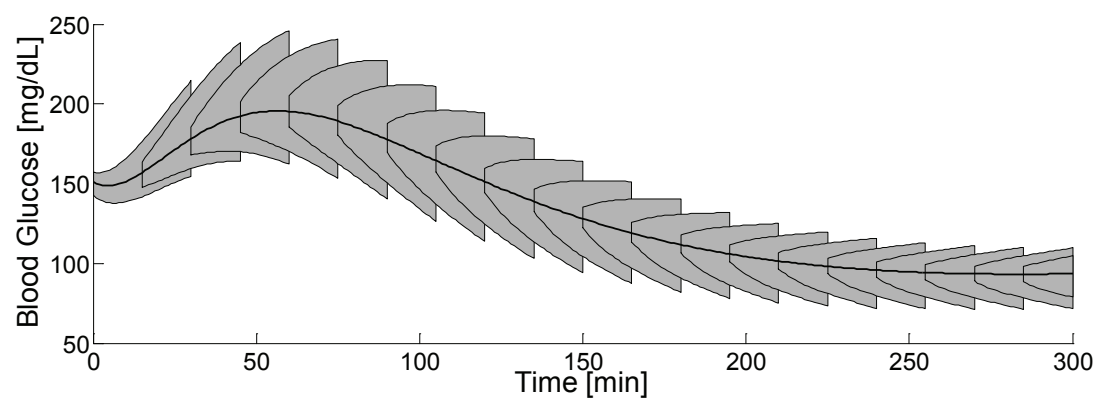

Figure 6.9: Solution envelope for 30-minute-ahead predictions with glucose measurements every 15 minutes.

In the case of short-term predictions (Figure 6.9), the solution envelope includes all the possible responses for the glucose level as expected. As the glucose measurements are performed every 15 minutes, the range of the glucose envelope is much smaller than in the long-term predictions. If the glucose measurements include less uncertainty, or if they are performed with 
a higher frequency, the range of the glucose envelope will be reduced even more. Observe that the computed glucose range is bigger around the glucose peak value and it reduces as euglycemia is reached again. This is due to the high influence of the uncertainty induced by the meal intake, which vanishes when the equilibrium point is attained. Compared to traditional approaches, a worst-case analysis can be carried out to produce more robust and safer decisions at each sample period.

\subsection{Discussion and conclusion}

The simulations carried out in the previous section are illustrative of the computational needs in a variety of problems where long- or short-term glucose predictions are used. The computation of tight glucose envelopes for longterm predictions is important for the parameter identification [12], to develop new insulin pump therapies using set-inversion techniques [73], and to perform insulin dosage optimisation [26] minimising a compound index of hypoglycemia and hyperglycemia risk. These tools are fundamental in reducing the risks of hyperglycemic and hypoglycemic episodes, which can be deleterious for patients health. Furthermore, tight glucose envelopes for short-term predictions may produce more robust and safer insulin infusion algorithms, including robust fault detection [1] or Model Predictive Control [39]. Consideration of intra-patient variability into the simulation allows to increase the robustness of these methodologies, yielding to safer systems.

In this work, a monotonicity and critical points analysis of all the model parameters has been proven as a successful tool to compute solution envelopes of the glucose-insulin model considering parametric uncertainty. Nevertheless, as not all the compartments and parameters of the model satisfy the monotonicity conditions, an upper bounding model and a lower bounding model have been considered, which satisfy the monotonicity conditions, to compute a tight glucose envelope for the original glucose-insulin model.

A preceding contribution by Calm et al. [11] has tackled the same problem. However, some model parameters cannot be considered uncertain, which makes not possible a direct comparison with the proposed method.

Finally, one of the strengths of the method proposed is that, although it has been applied to compute tight solution bounds of the glucose-insulin model developed by Hovorka et al. [39], it can also be applied to other glucose-insulin models, or even for compartmental models that mimic other type of processes. In addition, the method computes guaranteed bounds with few overestimation and low computational cost, which make it suitable for real-time computation. The limitation of this approach is that, as the dynamic model structures differ, each model has to be analysed independently. Complexity of the analysis of critical points (when required) will also increase with the complexity of the model. 
In conclusion, monotone systems approach is an efficient tool to compute tight solution envelopes for glucose-insulin models under intra-patient variability. These tools may foster the development of more robust and safe algorithms for glycemic control in type 1 diabetes. 



\title{
Real-time estimation of plasma insulin concentration
}

\author{
The observer, when he seems to himself to \\ be observing a stone, is really observing the \\ effects of the stone upon himself.
}

Bertrand Russell

\subsection{Introduction}

Continuous glucose monitors can measure interstitial glucose concentration in real-time for the artificial pancreas. These closed-loop glucose control systems use an insulin feedback to maintain plasma glucose concentration within a narrow and safe range, and thus to avoid health complications. As insulin is administered subcutaneously, there is a big delay between the insulin therapy action and its effect in blood glucose. For this reason, it is crucial to know the levels of blood insulin to predict short-term glucose concentrations.

As it is not possible to measure plasma insulin concentration in real-time, insulin models have been used in literature to estimate them. Nevertheless, the significant inter- and intra-patient variability of insulin absorption jeopardizes the accuracy of these estimations. In order to reduce these limitations, the aim of this chapter is to perform a real-time estimation of plasma insulin concentration from continuous glucose monitoring.

Hovorka's glucose-insulin model has been incorporated in an Extended Kalman Filter in which different selected time-variant model parameters have been considered as extended states. The observability of the original Hovorka's model and of several extended models has been evaluated by their Lie derivatives. This methodology has been evaluated with an in-silico study with 
100 patients with Type 1 diabetes during 25 hours. Furthermore, it has been also validated using clinical data from 12 insulin pump patients with Type 1 diabetes who underwent four mixed meal studies. Real-time insulin estimations have been compared to plasma insulin measurements to assess performance showing the validity of the methodology here used in comparison with that formerly used for insulin models. Hence, real-time estimations for plasma insulin concentration based on subcutaneous glucose monitoring can be beneficial for increasing the efficiency of control algorithms for the artificial pancreas.

\subsection{Extended Kalman filter}

Mathematical models try to mimic real-life processes, which are characterized by their variability. Uncertainty can arise due to the lack of information we have about the process itself, as it is not possible to measure efficiently all its indicators in real-time. As a consequence, we may have noisy measurements about some states, and a total deficit of information about the rest of the model. However, the limited set of measurements can be overcome with the conjunction of a model and a state observer [22].

One of the most popular observers is the Kalman Filter [22, 44], that can be used in continuous or discrete time, or combining discrete measurements in continuous time [17]. It is a stochastic filter that allows the estimation of the states of a system based on a linear state-space model. This observer estimates system states in a two-step process: the prediction and the correction steps. In the prediction step, the Kalman filter estimates the current state variables. Once a new measurement (with noise) is observed, the state estimations are updated using a weighted process [22, 44]. The extended Kalman filter (EKF) uses a local linearisation to extend the scope of the Kalman filter to systems described by non-linear ordinary differential equations [59, 43].

High variability processes may be represented by systems with noise in their dynamic and measurements. These systems can be expressed by:

$$
\begin{array}{ll}
\dot{x}(t)=f(x(t), u(t))+w(t), & w(t) \sim N(0, Q(t)) \\
z(t)=h(x(t))+v(t), & v(t) \sim N(0, R(t))
\end{array}
$$

where $h(x(t))$ denotes the measurement function and $z(t)$ is the output of the model. Furthermore, $w(t)$ and $v(t)$ represent the process and the observation noises, while $Q(t)$ and $R(t)$ denote the variances of these noises, respectively.

The estimation of the system state vector $x(t)$ is represented by $\hat{x}(t)$ :

$$
\dot{\hat{x}}(t)=f(\hat{x}(t), u(t))+K(t)(z(t)-h(\hat{x}(t)))
$$

where $K(t)$ is the gain matrix. 
The linearized transition matrix, $F$, with elements defined by $f(x(t), u(t))$, is given by:

$$
F(t)=\left.\frac{\partial f}{\partial x}\right|_{\hat{x}(t), u(t)},
$$

with the covariance matrix, $P$, forward in time, that is:

$$
\dot{P}(t)=F(t) P(t)+P(t) F(t)^{\top}-K(t) H(t) P(t)+Q(t)
$$

where $H(t)$ is the measurement matrix:

$$
H(t)=\left.\frac{\partial h}{\partial x}\right|_{\hat{x}(t)} .
$$

Finally, the gain matrix is updated:

$$
K(t)=P(t) H(t)^{\top} R(t)^{-1}
$$

However, it is not always possible to estimate all the system states from a limited set of measurements. Observability analysis proves if the model states in $x(t)$ can be theoretically estimated from measurements of $z(t)$. This analysis can be assessed by the Lie derivatives [28, 38] of the glucose-insulin model. The Lie derivative of $h(x)$ with respect to $f(x, u)$ is defined as $L_{f(x, u)} h(x)=$ $\nabla h(x) \cdot f(x, u)$, and it is calculated recursively, as follows:

$$
\begin{aligned}
& L_{f(x, u)}^{i} h(x)=L_{f(x, u)}\left(L_{f(x, u)}^{(i-1)} h(x)\right) \quad \text { if } i=1,2, \ldots \\
& L_{f(x, u)}^{0} h(x)=h(x)
\end{aligned}
$$

A function $h(x)$ is considered observable if the following Jacobian matrix

$$
\frac{\partial}{\partial x}\left[\begin{array}{c}
h(x) \\
L_{f(x, u)} h(x) \\
\cdots \\
L_{f(x, u)}^{n-1} h(x)
\end{array}\right]
$$

has rank $n$, where $n$ is the system order.

\subsection{Real-time estimation of plasma insulin}

Since insulin cannot be measured in real-time, insulin models have been used to estimate such concentration [67, 83]. However, variability of pharmacokinetic dynamics jeopardizes the accuracy of the estimation [20]. This variability is due to the distribution of the injection and the characteristics of the subcutaneous tissue, and it is basically focused on two parameters: time-to-peak plasma insulin concentration and metabolic clearance rate of insulin [33, 72]. This question limits the performance of any artificial pancreas potentially, 
leading to either over- or under-insulinization and posing patient's safety at risk. Effective closed-loop glucose control requires the design of robust strategies integrating patient's variability into tools for an accurate plasma insulin estimation.

Kalman Filters have been used to enhance the accuracy of continuous glucose monitors [25, 47] in humans. Furthermore, they have been used to estimate insulin concentration from intravenous glucose tolerance tests (IVGTT) in non-diabetic humans [23], and from subcutaneous (SC) glucose measurements in diabetic and non-diabetic pigs [24].

In this section, several approaches for an estimation of plasma insulin concentration in real-time are presented. A specific model has been chosen to illustrate these techniques, although they could be easily extended to any other model.

\subsubsection{Estimation from an insulin model}

A common approach in literature to estimate plasma insulin concentration is to simulate the insulin intakes in an insulin model [67, 83]. The simulation of an insulin model acts like an open-loop, as the glucose measurements are not used to adjust the states or the parameters of the system. Nevertheless, these adjustments could be necessary, especially in processes with high variability. In general, this system can be represented by:

$$
\dot{x}(t)=f(x(t), u(t))
$$

where $u(t)$ is the input of the model, $x(t)$ is the system state vector, and $f(x(t), u(t))$ defines the system's dynamics.

The insulin model considered in this work is the model developed by Hovorka et al. [39], described in Section 6.2 System's dynamics $f(x(t), u(t))$ are given by the subcutaneous insulin absorption model (Section 6.2.2), while the system's state vector $x(t)$ is given by:

$$
x(t)=\left[S_{1}, S_{2}, I\right] .
$$

\subsubsection{State observation of a glucose-insulin model}

The glucose-insulin model developed by Hovorka et al. [39] describes the blood glucose dynamics based on carbohydrate ingestion and insulin infusion. However, only interstitial glucose concentration can be measured by SC glucose monitors. Therefore, a new equation that describes the relation between blood glucose concentration and interstitial glucose concentration (mmol/L) 47, 89] is added in the system:

$$
\dot{I G}(t)=\frac{1}{\tau}\left(\frac{Q_{1}(t)}{V_{G}}-I G(t)\right)
$$


where $V_{G}(0.16 *$ Patient_weight $L)$ denotes the volume of the accessible compartment, and $\tau(16 \mathrm{~min})$ represents the time delay.

The system state vector $x(t)$ is given by:

$$
x(t)=\left[S_{1}, S_{2}, I, x_{1}, x_{2}, x_{3}, Q_{1}, Q_{2}, I G\right]
$$

Note that $U_{G}(t)$ is not included in the state vector since it is explicitly expressed in Section 6.2.1, and not as a dynamical equation. Function $f(x(t), u(t))$ is described by the dynamical equations exposed in Section 6.2. The measurement matrix $H(t)$ is given by:

$$
H(t)=\left.\frac{\partial h}{\partial x}\right|_{\hat{x}(t)}=[0,0,0,0,0,0,0,0,1]
$$

as only interstitial glucose concentration $(I G)$ can be measured by SC glucose monitors, i.e., $h(x(t))=I G(t)$.

In this approach, the variances of the process and observation noises $(Q(t)$ and $R(t)$, respectively) have been heuristically adapted to:

$$
\begin{aligned}
& Q(t)=\operatorname{diag}\left(20,20,0.01,10^{-6}, 10^{-8}, 10^{-5}, 1,1,1\right) \\
& R(t)=0.45 \mathrm{mmol} / \mathrm{L} \quad[=8 \mathrm{mg} / \mathrm{dl}] .
\end{aligned}
$$

\subsubsection{State observation of an extended model}

The main problem with simulations and state observers is that they are often developed assuming perfect knowledge of the system dynamics, in particular of the parameter values. However, this is not true due to the high variability of the processes, which leads to uncertain parameters.

State observers can be used to estimate these uncertain parameters by building new extended models in which these parameters are considered as new states with no dynamics [22, 24]. For this reason, extended Hovorka's models have been defined such that different parameters have been considered as extended states of the model:

$$
\frac{d}{d t}\left[\begin{array}{l}
x(t) \\
p(t)
\end{array}\right]=\left[\begin{array}{c}
\left.f(x(t), u(t))\right|_{p=p(t)} \\
0
\end{array}\right]
$$

The parameters with the greatest impact in plasma insulin concentration are $k_{e}$ (plasma insulin elimination rate) and $t_{\max , I}$ (time to maximum insulin absorption), as seen in literature [33, 72] and it has been verified experimentally in this work (modification on other model parameters do not produce significant variations in insulin estimation). Hence, the main interest is to estimate these parameters, so they have been selected as uncertain states in extended Hovorka's models, where one or both parameters have been considered as extended states. The observability of these models has been evaluated by their Lie derivatives. 


\section{$\begin{array}{ll}\text { Initial } & E K F \quad \begin{array}{l}\text { Value at } \\ \text { values }\end{array}\end{array}$}
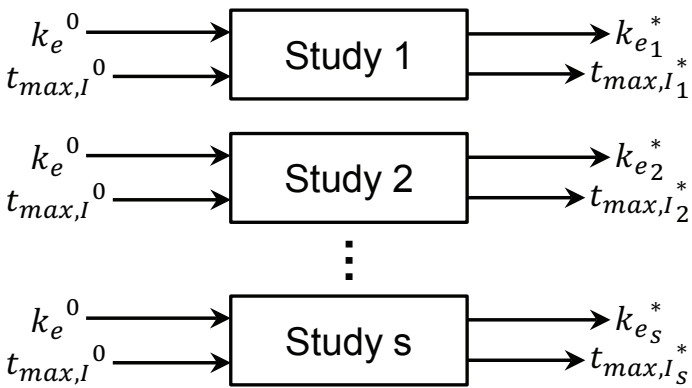

Figure 7.1: Diagram of the adjustment of uncertain parameters.

Then, the extended models have been incorporated in an EKF, such that the parameters $k_{e}$ and/or $t_{\max , I}$ are adapted along time, as seen in Figure 7.1 During each study, the system states are estimated based on punctual measurements of plasma glucose concentration. The initial value of the uncertain parameters $\left(k_{e}^{0}\right.$ and $\left.t_{\max , I}{ }^{0}\right)$ has been set at their nominal value [39] for all the studies. The value of these parameters at the end of the study is $k_{e i}^{*}$ and $t_{\max , I_{i}^{*}}, i=1, \ldots, s$, where $s$ is the number of studies. The variance of the process noise of the extended states $k_{e}$ and $t_{\max , I}$ has been added to the matrix $Q(t)$, exactly the heuristically selected values $10^{-4}$ and 1 , respectively.

\subsection{Validation of insulin estimations}

In this section, insulin estimations are validated in both an in-silico study and using real data from patients.

\subsubsection{In-silico validation}

In-silico patients with Type 1 diabetes have been simulated during 1500 minutes (25 hours), including a meal of $300 \mathrm{mmol}$ ( $54 \mathrm{grams}$ ) of glucose every 300 minutes, for a total of five meals. Five equal meals do not represent a realistic scenario, but it allows to analyse the behaviour of the estimations with varying parameters in the same scenario. Each meal is compensated with a bolus of $2300 \mathrm{mU}$ of insulin. Furthermore, each patient receives a basal insulin infusion of $10 \mathrm{mU} / \mathrm{min}$. During the simulation, the values of the parameters $k_{e}$ and $t_{\max , I}$ vary along time based on their variability. The rest of the parameters assume their nominal value.

Real-time insulin estimations of 100 in-silico patients have been computed based on interstitial glucose measurements every 15 minutes. Insulin esti- 
mations $(\hat{z})$ have been compared to plasma insulin simulation $(z(t))$ to assess the methodology introduced. The performance of the different approaches has been quantitatively evaluated by computing the Root Mean Square Error (RMSE) and the Mean Absolute Relative Deviation (MARD):

$$
\begin{aligned}
& \text { RMSE }=\sqrt{\frac{1}{m} \sum_{t=1}^{m}\left(z_{t}-\hat{z}_{t}\right)^{2}} \\
& \text { MARD }=\frac{1}{m} \sum_{t=1}^{m} \frac{\left|z_{t}-\hat{z}_{t}\right|}{z_{t}}
\end{aligned}
$$

where $m$ is the number of measurements.

\subsubsection{Validation with real data}

State observers need several data values to adapt the system states (including the extended parameters). However, continuous glucose monitors only provide a new glucose concentration value every 1-15 minutes. Thus, the state observer is not able to adapt the system states during the first minutes of the experiment. The initial value of the system states is crucial during the first minutes of the insulin estimation, as the EKF needs some time to be able to perform an accurate estimation. If the values are under- or over-estimated, large errors may be produced in an insulin estimation. For this reason, changing the initial values of the extended states, i.e. parameters $k_{e}$ and $t_{\max , I}$, for more realistic values is proposed.
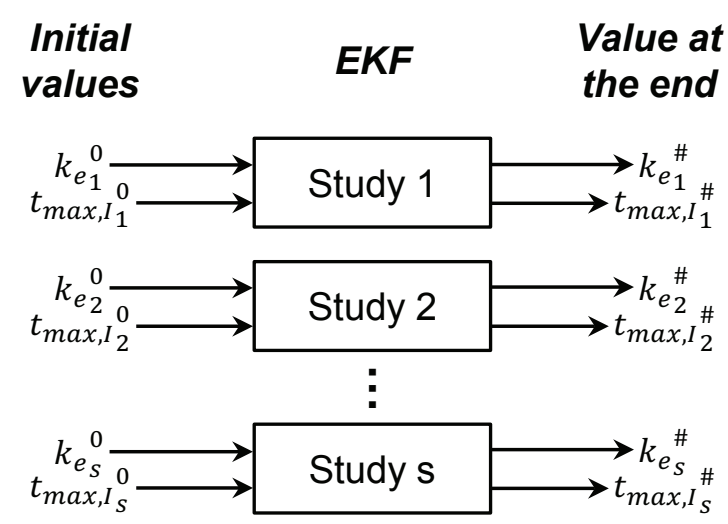

Figure 7.2: Diagram of the adjustment of uncertain parameters.

These new initial values are determined by a cross-validation of the estimated values of the uncertain parameters at the end of the rest of the studies: 


$$
\begin{aligned}
k_{e i}^{0} & =\frac{1}{s-1} \sum_{j=1}^{s}\left(1-\delta_{i, j}\right) k_{e_{j}^{*}}^{*} \\
t_{\max , I_{i}^{0}} & =\frac{1}{s-1} \sum_{j=1}^{s}\left(1-\delta_{i, j}\right) t_{\max , I_{j}^{*}}^{*}
\end{aligned}
$$

where $s$ is the number of studies, and the function $\delta_{i, j}$ is defined as:

$$
\delta_{i, j}= \begin{cases}1 & \text { if } i=j \\ 0 & \text { if } i \neq j\end{cases}
$$

These new initial values are represented in Figure 7.2. During each study, the value of the uncertain parameters $k_{e}$ and $t_{\max , I}$ is adapted through time.

Insulin estimations have been compared using real data from 12 insulin pump patients with Type 1 diabetes who underwent four mixed meal studies (9 women; $41.8 \pm 7.3$ years old; diabetes duration $20.2 \pm 10.3$ years; body mass index: $25.1 \pm 2.8 \mathrm{~kg} / \mathrm{m}^{2}$ ) [74]. The performance of insulin estimations has been evaluated by a comparison with plasma insulin measurements, computing RMSE (7.3) and MARD (7.4).

\subsection{Results}

Different estimations of plasma insulin concentration have been performed according to the approaches presented in the previous sections. All the simulations have been executed with Matlab software (version R2013a) using an Intel(R) Core i7-3770K 3.5 GHz Pentium(R) processor. The obtained results are included hereinafter and commented with detail in the discussion section.

\subsubsection{Observability}

The observability of the original Hovorka's model and of the extended models with the following state vectors:

$$
\begin{aligned}
& x(t)=\left[S_{1}, S_{2}, I, x_{1}, x_{2}, x_{3}, Q_{1}, Q_{2}, I G\right] \\
& x(t)=\left[S_{1}, S_{2}, I, x_{1}, x_{2}, x_{3}, Q_{1}, Q_{2}, I G, k_{e}\right] \\
& x(t)=\left[S_{1}, S_{2}, I, x_{1}, x_{2}, x_{3}, Q_{1}, Q_{2}, I G, t_{\max , I}\right] \\
& x(t)=\left[S_{1}, S_{2}, I, x_{1}, x_{2}, x_{3}, Q_{1}, Q_{2}, I G, k_{e}, t_{\max , I}\right]
\end{aligned}
$$

has been evaluated by their Lie derivatives [28, 38]. In all the cases, the jacobian matrices (7.2) have full-rank (rank 9, 10, 10 and 11, respectively). Therefore, all the systems are observable, and it is theoretically possible to estimate all the states from $I G(t)$ interstitial glucose measurements.

\subsubsection{In-silico results}

In the original Hovorka's model all the model parameters are considered constant along time. However, during the in-silico simulation, parameters $k_{e}$ and 


\begin{tabular}{lccc}
\hline Model & RMSE $(m U / L)$ & MARD $(\%)$ & Computational Time (s) \\
\hline Hovorka's Insulin Model & $5.6 \pm 2.9$ & $50 \% \pm 26 \%$ & $3 \cdot 10^{-6} \mathrm{~s}$ \\
Original Hovorka's EKF & $5.5 \pm 2.0$ & $51 \% \pm 26 \%$ & $0.095 \mathrm{~s}$ \\
$k_{e}$ Parameter EKF & $3.2 \pm 1.7$ & $19 \% \pm 5 \%$ & $0.098 \mathrm{~s}$ \\
$t_{\max , I}$ Parameter EKF & $3.9 \pm 1.0$ & $40 \% \pm 18 \%$ & $0.097 \mathrm{~s}$ \\
$k_{e}-t_{\max , I}$ Parameters EKF & $2.4 \pm 0.6$ & $16 \% \pm 4 \%$ & $0.100 \mathrm{~s}$ \\
\hline
\end{tabular}

Table 7.1: Results for in-silico estimation of plasma insulin concentration applying different approaches. The results shows the RMSE and MARD errors committed (and their standard deviation) in the estimation of plasma insulin concentration with respect to insulin simulation.

$t_{\max , I}$ vary along time. These differences lead to large values for RMSE and MARD in the plasma insulin estimation, as seen in Table 7.1. The estimations performed through the EKF are represented with a solid line in Figure 7.3 . while the square dots represent the values of the in-silico simulation.
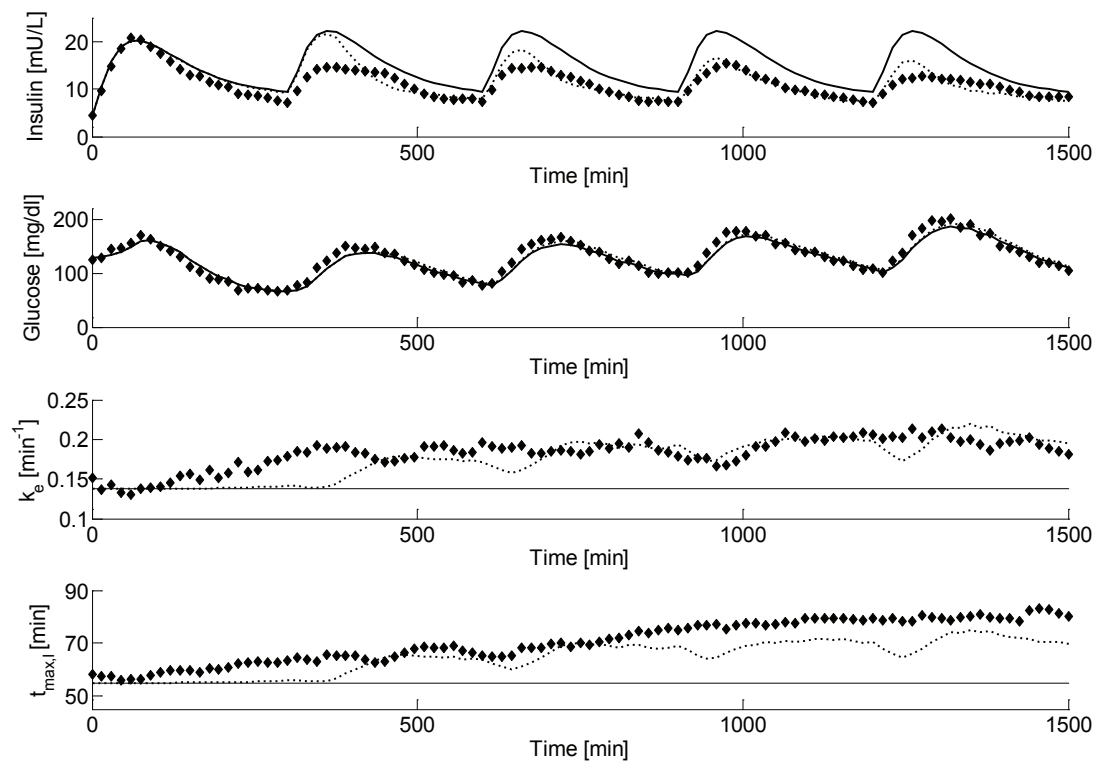

Figure 7.3: Example of in-silico estimations of plasma insulin concentration. The square dots represent measurements from the in-silico simulation, while the lines represent several estimation approaches: Original Hovorka EKF (solid line) and $k_{e}-t_{\max , I}$ Parameters EKF (dotted line).

The model parameters $k_{e}$ and $t_{\max , I}$ (one or both) can be considered as extended states in the Hovorka's model, and incorporated in an EKF. The results obtained are represented in Table 7.1, showing that the best results are achieved when both parameters are considered as extended states. This estimation is shown with a dotted line in Figure 7.3 . This approach improves 
significantly $(p<0.001)$ the results obtained with the simulation of Hovorka's insulin model. The computational time for each approach is shown in Table 7.1 .

\subsubsection{Results with real data}

Simulation of Hovorka's insulin model overestimates the plasma insulin concentration in all the patients, as parameters $k_{e}$ and $t_{\max , I}$ may be underestimated. Almost the same overestimation is obtained when the estimation of all the system states is performed through an EKF, represented with a solid line in Figure 7.4, while the square dots represent real measurements of plasma insulin and glucose concentration. The plasma insulin overestimation leads to large values for RMSE and MARD, as shown in Table 7.2 Table 7.3 shows the results when an outlier patient is omitted.
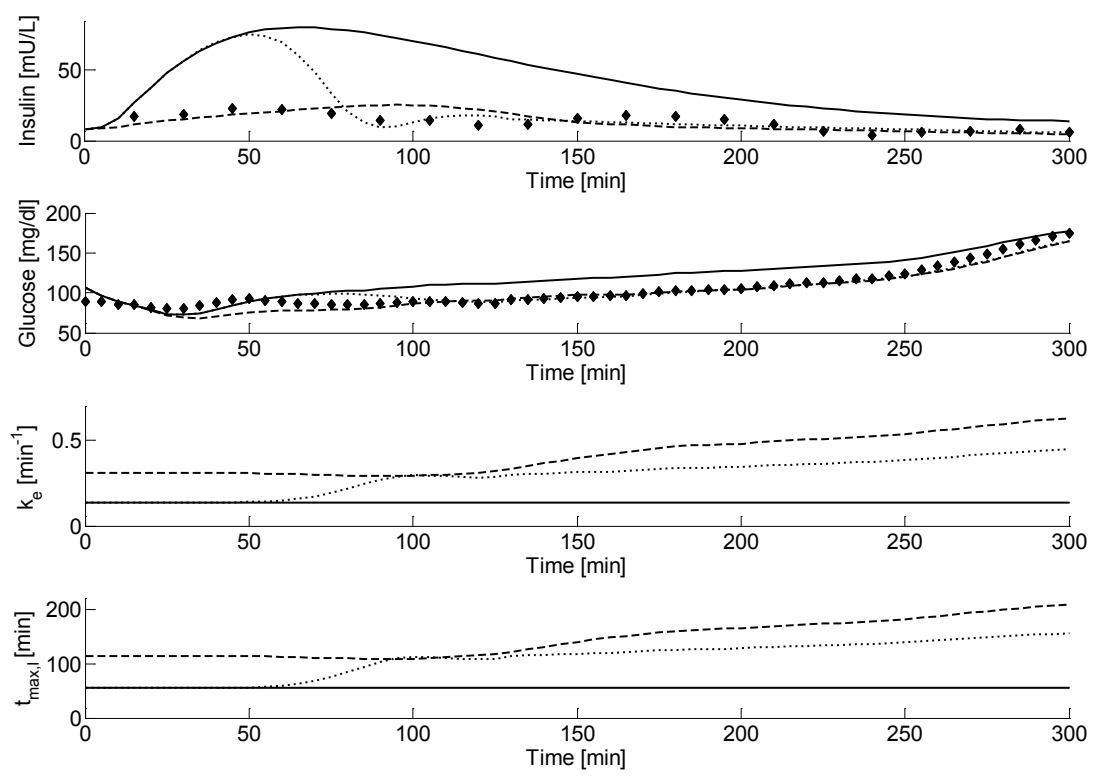

Figure 7.4: Example of estimations of plasma insulin concentration with real data. The square dots represent real measurements, while the lines represent several estimation approaches: Original Hovorka EKF (solid line), $k_{e}-t_{m a x, I}$ Parameters EKF (dotted line) and Cross-Validation $k_{e}-t_{\max , I} \operatorname{EKF}$ (dashed line).

In the above estimations, the parameters of Hovorka's model have been considered constant along time. However, the model parameters $k_{e}$ and $t_{\max , I}$ can be adapted if they are considered as extended states in the Hovorka's model. Each extended model has been incorporated in an EKF. The best results are obtained when both parameters are considered as extended states, 


\begin{tabular}{lccc}
\hline Model & RMSE $(m U / L)$ & MARD (\%) & Computational Time (s) \\
\hline Hovorka's Insulin Model & $24.5 \pm 16.5$ & $179 \% \pm 158 \%$ & $3 \cdot 10^{-6} \mathrm{~s}$ \\
Original Hovorka's EKF & $24.4 \pm 16.4$ & $177 \% \pm 156 \%$ & $0.095 \mathrm{~s}$ \\
$k_{e}$ Parameter EKF & $15.3 \pm 7.6$ & $87 \% \pm 90 \%$ & $0.098 \mathrm{~s}$ \\
$t_{\max , I}$ Parameter EKF & $18.7 \pm 11.1$ & $133 \% \pm 134 \%$ & $0.097 \mathrm{~s}$ \\
$k_{e}-t_{\max , I}$ Parameters EKF & $14.9 \pm 7.7$ & $85 \% \pm 100 \%$ & $0.100 \mathrm{~s}$ \\
Cross-Valid. $k_{e}-t_{\max , I}$ EKF & $6.6 \pm 3.9$ & $49 \% \pm 72 \%$ & $0.100 \mathrm{~s}$ \\
\hline
\end{tabular}

Table 7.2: Results for real data estimation of plasma insulin concentration applying different approaches. The results shows the RMSE and MARD errors committed (and their standard deviation) in the estimation of plasma insulin concentration for the 12 patients with respect to real insulin measurements.

\begin{tabular}{lccc}
\hline Model & RMSE $(m U / L)$ & MARD $(\%)$ & Computational Time (s) \\
\hline Hovorka's Insulin Model & $23.5 \pm 14.9$ & $145 \% \pm 103 \%$ & $3 \cdot 10^{-6} \mathrm{~s}$ \\
Original Hovorka's EKF & $23.4 \pm 14.9$ & $141 \% \pm 101 \%$ & $0.095 \mathrm{~s}$ \\
$k_{e}$ Parameter EKF & $14.7 \pm 6.8$ & $66 \% \pm 25 \%$ & $0.098 \mathrm{~s}$ \\
$t_{\max , I}$ Parameter EKF & $17.8 \pm 9.9$ & $101 \% \pm 66 \%$ & $0.097 \mathrm{~s}$ \\
$k_{e} t_{\max , I}$ Parameters EKF & $14.3 \pm 6.9$ & $61 \% \pm 28 \%$ & $0.100 \mathrm{~s}$ \\
Cross-Valid. $k_{e}-t_{\max , I}$ EKF & $6.4 \pm 3.9$ & $33 \% \pm 14 \%$ & $0.100 \mathrm{~s}$ \\
\hline
\end{tabular}

Table 7.3: Results for real data estimation of plasma insulin concentration applying different approaches. The results shows the RMSE and MARD errors committed (and their standard deviation) in the estimation of plasma insulin concentration for 11 patients (excluding the outlier patient) with respect to real insulin measurements.

as seen in Tables 7.2 and 7.3. These results are represented in Figure 7.4 with a dotted line. This approach improves significantly $(p<0.001)$ the results obtained with the simulation of Hovorka's insulin model.

Finally, the estimations further improve after a cross-validation of the estimated values at the end of the studies of the uncertain parameters $k_{e}(0.32 \pm$ $\left.0.10 \mathrm{~min}^{-1}\right)$ and $t_{\max , I}(115 \pm 33 \mathrm{~min})$, as shown in Tables 7.2 and 7.3 and in Figure 7.4 with a dashed line. The computational time for each approach is shown in Tables 7.2 and 7.3 .

\subsection{Discussion and conclusion}

The experiments carried out in the previous section illustrate several approaches to estimate plasma insulin concentration through SC interstitial glucose measurements in Hovorka's model. This model has been chosen to illustrate the techniques, although they could be extended to other models.

In both the in-silico and the real data studies, a large error is committed when the Hovorka's insulin model is simulated (a common approach in literature), as seen in Tables 7.1, 7.2 and 7.3 In this model, a perfect knowledge of the system parameters is assumed, as they remain constant along time. Parameters $k_{e}$ and $t_{\max , I}$ may be underestimated, producing an overestimation 
on insulin concentration. On the other hand, insulin estimation would be underestimated if these parameters were overestimated. Hence, it is crucial to adjust parameters $k_{e}$ and $t_{\max , I}$ to perform an estimation of plasma insulin concentration.

Insulin estimation is significantly improved when parameters $k_{e}$ and $t_{\max , I}$ are considered as extended states. Once the parameters are adjusted, plasma insulin estimations are also adjusted. Consequently, time variations in both parameters may be responsible, at least in part, of intra- and inter-patient variability in insulin stacking, leading to inaccurate plasma insulin estimations. When these parameters are considered as extended states, it is possible to perform individualized estimations of plasma insulin concentration.

However, the EKF need several glucose measurements to adapt the system parameters. In most of real-data cases, the nominal values of parameters $k_{e}$ and $t_{\max , I}$ [39] are underestimated, producing a large overestimation of plasma insulin concentration. After the cross-validation, the errors decrease (Tables 7.2 and 7.3), specially those during the first minutes of the estimation (Figure 7.4). When this final approach is applied, both RMS and MARD errors are reduced in a $73 \%$ with respect to the simulation of Hovorka's insulin model. Therefore, this approach is proposed for the estimation of plasma insulin concentration through interstitial glucose measurements.

Observing Figure 7.4 it may seem that once the parameters are adapted, they do not vary much along time. However, these parameters may vary significantly during long-term estimations. In Figure 7.3 , the initial values of parameters $k_{e}$ and $t_{\max , I}$ are similar to the one used in the estimations. Both the Original Hovorka's EKF and the $k_{e}-t_{\max , I}$ EKF accomplish an accurate estimation of insulin concentration during the first meal. However, once the parameters values have varied, only the $k_{e}-t_{\max , I}$ EKF is able to adapt itself and perform much better estimations of plasma insulin concentration than the Original Hovorka's EKF. Hence, in long-term estimations it is important to consider $k_{e}$ and $t_{\max , I}$ parameters as extended states to adapt them along time.

Nevertheless, there is still a significant error, but it is not totally inevitable. This is, in part, a consequence of the error committed in the interstitial glucose measurements, as the SC continuous glucose monitors do not accomplish accurate measurements [4]. Errors in plasma insulin estimations may decrease if continuous glucose monitors improve their performance. Furthermore, in exception of $k_{e}$ and $t_{\max , I}$, the rest of the parameters of Hovorka's model assume their nominal value during the estimations. However, some of these parameters, such as the insulin sensitivity, have large variability. An individualization of these parameter values may improve plasma insulin estimations.

Most of the error committed (Table 7.2) is accumulated in a single outlier patient, in which insulin measurements do not represent reliable values. If this outlier patient is not considered in the calculations, the metrics improve 
significantly, specially the MARD value (Table 7.3).

Previous studies have carried out insulin estimations in non-diabetic humans after intravenous glucose injections [23], and in diabetic and non-diabetic pigs [24]. In this work, the behaviour of diabetic human patients after a meal ingestion has been analysed, based on SC glucose measurements. In conclusion, this study approaches us to the real operation of the artificial pancreas, and improves the performance of real-time estimations for plasma insulin concentration, with respect to insulin models used in literature. From these results based on SC glucose monitoring, the proposed approach may be beneficial for increasing the efficiency and safety of control algorithms for the artificial pancreas.

In this work, Hovorka's glucose-insulin model has been validated as part of an insulin observer system, based on subcutaneous glucose measurements. A natural extension of this work would be the inclusion of this model in an interval observer, in which the methods exposed in the previous chapters can be applied. However, this combination is not straightforward, as interval observers require hard restrictions on the observer monotonicity that are only satisfied in selected systems [31]. As a consequence, this problem is remained open as a future research line. 



\section{Conclusions of this thesis}

Lo urgente puede esperar.

Lo importante no debería.

Anónimo

This thesis presents new approaches and methodologies in order to overcome some of the problems of model uncertainty: the computation of guaranteed solution envelopes and real-time state estimation. The main contributions of this work are summarized on the particular section Discussion and conclusion that closes each chapter.

These are the main problems tackled in this thesis:

1. The Computation of guaranteed solution envelopes is generally performed in literature by an orthant monotonicity analysis approach. When the monotonicity conditions are satisfied, it is possible to compute the exact solution bounds. Otherwise, some overestimation is obtained. In order to overcome (or minimize) this problem, several approaches have been developed to eliminate or reduce the overestimation.

1.1. Common structures for biological models have been analysed in Chapter 3 (parallel inputs models) and Chapter 4 (in-series models). Solution envelopes have been computed using several methods, such as model reduction, analysis of critical points, change of variables or application of differential inequalities. These techniques have been applied in conjunction to the classical monotonicity analysis for the computation of tight guaranteed solution envelopes, minimizing the overestimation.

1.2. A novel approach based on cone monotonicity is introduced in Chapter 5, which is specially useful when the classical orthant monotonicity conditions are not satisfied. This new method is based on monotonicity with respect to an ordering induced by an arbitrary cone, not necessarily an orthant. After a cone monotonicity analysis, the input space used for computation of guaranteed solution envelopes is reduced to just the boundary of the initial conditions 
space. Therefore, the overestimation produced is reduced significantly.

1.3. Application: Prediction of blood glucose concentration in subjects with type 1 diabetes. Intra-patient variability has been considered in all the parameters and initial conditions of the model. Tight glucose envelopes that bound all possible glycemic responses have been computed in Chapter 6

2. Real-time estimation of uncertain states and parameters is performed using state observers and partial measurements from the process.

2.1. Application: Real-time insulin concentration estimation. In Chapter 7 an Extended Kalman Filter has been incorporated in a model in which different selected time-variant model parameters have been considered as extended states. Plasma insulin concentration is estimated in real-time from noisy measurements of a continuous glucose monitor.

\section{Contributions}

Contributions related and contained in this thesis have been made available through the following publications listed below:

\section{Journal articles}

- Diego De Pereda, Sergio Romero-Vivo, Beatriz Ricarte, Jorge Bondia. On the computation of output bounds for compartmental in-series models under parametric uncertainty. Biomath 1, 2 (2012), 1210043.

- Diego De Pereda, Sergio Romero-Vivo, Beatriz Ricarte, Jorge Bondia. On the prediction of glucose concentration under intra-patient variability in type 1 diabetes: A monotone systems approach. Computer Methods and Programs in Biomedicine 108 (2012), 993-1001.

- Diego De Pereda, Sergio Romero-Vivo, Jorge Bondia. On the computation of output bounds on parallel inputs pharmacokinetic models with parametric uncertainty. Mathematical and Computer Modelling 57, 7 (2013), 1760-1767.

- Diego De Pereda, Sergio Romero-Vivo, Beatriz Ricarte, Jorge Bondia. Guaranteed computation methods for compartmental in-series models under uncertainty. Computers $\mathcal{E}$ Mathematics with Applications 66, 9 (2013), 1595-1605. 


\section{Conference proceedings}

- Beatriz Ricarte, Sergio Romero-Vivo, Diego De Pereda, Jorge Bondia. Monotonicity-based guaranteed prediction for glucose control and supervision under intra-patient variability. In European Control Conference (2015).

\section{Conference posters and oral presentations}

- Diego De Pereda, Sergio Romero-Vivo, Jorge Bondia. On the computation of output bounds on parallel inputs pharmacokinetic models with parametric uncertainty. In Mathematical Modelling in Engineering $\mathcal{E} \mathrm{Hu}$ man Behaviour (2011).

- Diego De Pereda, Sergio Romero-Vivo, Beatriz Ricarte, Jorge Bondia. A new method for the prediction of glucose concentration under uncertainty in type 1 diabetes. In Advanced Technologies $\mathcal{E}$ Treatments for Diabetes (2012).

- Diego De Pereda, Sergio Romero-Vivo, Beatriz Ricarte, Jorge Bondia. Output bounds for compartmental in-series models under parametric uncertainty. In International Conference on Mathematical Methods and Models in Biosciences (2012).

- Diego De Pereda, Sergio Romero-Vivo, Beatriz Ricarte, Jorge Bondia. On generalized cooperative systems and the computation of their solution envelopes. In Mathematical Modelling in Engineering \& Human Behaviour (2012).

- Diego De Pereda, Sergio Romero-Vivo, Beatriz Ricarte, Jorge Bondia. Predictive Tools for Diabetes Management based on Cooperativity. In 4th International Symposium on Positive Systems: Theory and Applications (2012).

- Diego De Pereda, Sergio Romero-Vivo, Beatriz Ricarte, Paolo Rossetti, Javier Ampudia-Blasco, Jorge Bondia. Real-Time Estimation of Plasma Insulin Concentrations from SC Continuous Glucose Monitoring. In Diabetes Technology Meeting (2013).

- Diego De Pereda, Sergio Romero-Vivo, Beatriz Ricarte, Paolo Rossetti, Javier Ampudia-Blasco, Jorge Bondia. Real-time estimation of the InsulinOn-Board using the Extended Kalman Filter for glucose control in artificial pancreas. In Advanced Technologies \& Treatments for Diabetes (2014). 


\section{Future work lines}

The research lines exposed here may be continued following different work lines:

Solution envelopes for common biological structures has been computed in this thesis. A deep analysis of other model structures could bring more techniques to compute guaranteed solution envelopes in some specific situations. Furthermore, other approaches could be developed to avoid some of the limitations of the proposed methods, as the constant or impulse inputs restriction in parallel inputs structures.

General approaches to compute solution envelopes could be created. In this thesis, a novel approach based on monotonicity with respect to an ordering induced by an arbitrary cone has been exposed. This technique reduce the overestimation when the classical orthant monotonicity conditions are not satisfied. However, the system must be monotone with respect to any cone and it do not remove the overestimation completely. As a consequence, there is still place for improvement.

Interval observers for glucose-insulin systems could be developed applying the techniques developed for guaranteed computation. The main limitation of interval observers is that they require hard conditions that only selected systems satisfy. These systems have normally 2 or 3 equations only, while the glucose-insulin system created by Hovoka et al. has 9 differential equations. This research line is currently under development.

Application to more topics. The examples used in this thesis are from biological systems. In fact, most of them are glucose-insulin systems, applicable to the diabetes disease. However, the techniques exposed herein could be applied to any other dynamical system under uncertainty. 


\section{References}

The problem with quotes on the Internet is that it is hard to verify their authenticity.

Abraham Lincoln

[1] Armengol, J., Vehí, J., Sainz, M., Herrero, P., And Gelso, E. Squaltrack: a tool for robust fault detection. Systems, Man, and Cybernetics, Part B: Cybernetics, IEEE Transactions on 39, 2 (2009), 475-488.

[2] Auer, E., Rauh, A., Hofer, E. P., AND Luther, W. Validated modeling of mechanical systems with smartmobile: Improvement of performance by valencia-ivp. In Reliable Implementation of Real Number Algorithms: Theory and Practice. Springer, 2008, pp. 1-27.

[3] Bailey, N., ET AL. The mathematical theory of infectious diseases and its applications. Charles Griffin \& Company Ltd, 5a Crendon Street, High Wycombe, Bucks HP13 6LE., 1975.

[4] Barceló-Rico, F., Bondia, J., Díez, J. L., And Rossetti, P. A multiple local models approach to accuracy improvement in continuous glucose monitoring. Diabetes technology \& therapeutics 14, 1 (2012), 74-82.

[5] BECKER, K. L. Principles and practice of endocrinology and metabolism. Lippincott Williams \& Wilkins, 2001.

[6] Berman, A., And Plemmons, R. Nonnegative matrices in the Mathematical Sciences. Siam., 1994.

[7] BOCK, A., FRANÇOIS, G., AND GILLET, D. Estimating reliability of blood glucose concentration predictions in patients with type 1 diabetes mellitus. Tech. rep., Elsevier, 2014.

[8] Bonate, P. Pharmacokinetic-pharmacodynamic modeling and simulation. Springer Verlag, 2005.

[9] BONDAR, K. Some scalar difference inequalities. Applied Mathematical Sciences 5, 60 (2011), 2951-2956. 
[10] Bravo, J., Alamo, T., AND CAmacho, E. Robust mpc of constrained discrete-time nonlinear systems based on approximated reachable sets. Automatica 42, 10 (2006), 1745-1751.

[11] Calm, R., García-Jaramillo, M., Bondia, J., Sainz, M., AND Vehí, J. Comparison of interval and monte carlo simulation for the prediction of postprandial glucose under uncertainty in type 1 diabetes mellitus. Computer Methods and Programs in Biomedicine, 104, 3 (2011), 325-332.

[12] Calm, R., Sainz, M., Herrero, P., Vehi, J., And Armengol, J. Parameter identification with quantifiers. In Robust Control Design (2006), vol. 5, pp. 707-712.

[13] Capasso, V. Mathematical structures of epidemic systems, vol. 97. Springer Verlag, 1993.

[14] CARson, E., AND Cobelli, C. Modelling methodology for physiology and medicine. Academic Press, 2001.

[15] Chernin, E. Sir ronald ross, malaria, and the rewards of research. Medical history 32, 02 (1988), 119-141.

[16] Cobelli, C., Toffolo, G., And Ferrannini, E. A model of glucose kinetics and their control by insulin, compartmental and noncompartmental approaches. Mathematical biosciences 72, 2 (1984), 291-315.

[17] CRAssidis, J. L., AND JunKINS, J. L. Optimal estimation of dynamic systems. CRC press, 2011.

[18] De Pereda, D., Romero-Vivo, S., And Bondia, J. On the computation of output bounds on parallel inputs pharmacokinetic models with parametric uncertainty. Mathematical and Computer Modelling 57, 7 (2013), 1760-1767.

[19] de Pereda, D., Romero-Vivó, S., Ricarte, B., and Bondia, J. On the computation of output bounds for compartmental in-series models under parametric uncertainty. Biomath 1, 2 (2012), 1210043, http://dx.doi.org/10.11145/j.biomath.2012.10.043.

[20] De Pereda, D., Romero-Vivo, S., Ricarte, B., and Bondia, J. On the prediction of glucose concentration under intra-patient variability in type 1 diabetes: A monotone systems approach. Computer Methods and Programs in Biomedicine 108 (2012), 993-1001.

[21] De Pereda, D., Romero-Vivo, S., Ricarte, B., And Bondia, J. Guaranteed computation methods for compartmental in-series models under uncertainty. Computers \& Mathematics with Applications 66, 9 (2013), 15951605. 
[22] Dochain, D. State and parameter estimation in chemical and biochemical processes: a tutorial. Journal of process control 13, 8 (2003), 801-818.

[23] Eberle, C., AND Ament, C. The unscented kalman filter estimates the plasma insulin from glucose measurement. Biosystems 103, 1 (2011), 6772.

[24] Eberle, C., AND Ament, C. Real-time state estimation and long-term model adaptation: a two-sided approach toward personalized diagnosis of glucose and insulin levels. Journal of diabetes science and technology 6, 5 (2012), 1148-1158.

[25] Facchinetti, A., Sparacino, G., And Cobelli, C. Enhanced accuracy of continuous glucose monitoring by online extended kalman filtering. Diabetes technology $\mathcal{E}$ therapeutics 12, 5 (2010), 353-363.

[26] García-Jaramillo, M., Calm, R., Bondia, J., Tarín, C., And Vehí, $\mathrm{J}$. Insulin dosage optimization based on prediction of postprandial glucose excursions under uncertain parameters and food intake. Computer Methods and Programs in Biomedicine 105, 1 (2012), 61 - 69.

[27] Gardeñes, E., Sainz, M. A., Jorba, L., Calm, R., Estela, R., Mielgo, H., AND Trepat, A. Model intervals. Reliable Computing 7 (2001), 77-111.

[28] Gauthier, J., AND Bornard, G. Observability for any $\mathrm{u}(\mathrm{t})$ of a class of nonlinear systems. IEEE Transactions on Automatic Control 26, 4 (1981), 922-926.

[29] Gerich, J. E. Physiology of glucose homeostasis. Diabetes, Obesity and Metabolism 2, 6 (2000), 345-350.

[30] Godfrey, K., Arundel, P., Dong, Z., And Bryant, R. Modelling the Double Peak Phenomenon in pharmacokinetics. Computer Methods and Programs in Biomedicine, 104, 2 (2011), 62-69.

[31] Gouzé, J.-L., RAPAPORT, A., AND HADJ-SADOK, M. Z. Interval observers for uncertain biological systems. Ecological modelling 133, 1 (2000), 45-56.

[32] Haddad, W., Chellaboina, V., And Hui, Q. Nonnegative and compartmental dynamical systems. Princeton University Press, 2010.

[33] Haidar, A., Elleri, D., Kumareswaran, K., Leelarathna, L., Allen, J. M., Caldwell, K., Murphy, H. R., Wilinska, M. E., ACERINI, C. L., EVANS, M. L., ET AL. Pharmacokinetics of insulin aspart in pump-treated subjects with type 1 diabetes: reproducibility and effect of age, weight, and duration of diabetes. Diabetes care 36, 10 (2013), e173e174. 
[34] HALL, J. E., AND GuYTON, A. C. Textbook of medical physiology. Saunders, 2011.

[35] Hammersley, J., And Handscomb, D. Monte carlo methods. Taylor \& Francis, 1975.

[36] Heinemann, L. Variability of insulin absorption and insulin action. Diabetes technology \& therapeutics 4, 5 (2002), 673-682.

[37] Herbert, D., Elsworth, R., and Telling, R. The continuous culture of bacteria; a theoretical and experimental study. Journal of General Microbiology 14, 3 (1956), 601-622.

[38] Hermann, R., and Krener, A. J. Nonlinear controllability and observability. IEEE Transactions on automatic control 22, 5 (1977), 728-740.

[39] Hovorka, R., Canonico, V., Chassin, L., Haueter, U., MassiBenedetti, M., Federici, M., Pieber, T., Schaller, H., Schaupp, L., VERING, T., ET AL. Nonlinear model predictive control of glucose concentration in subjects with type 1 diabetes. Physiological measurement 25 (2004), 905.

[40] Hovorka, R., Shojaee-Moradie, F., Carroll, P., Chassin, L., Gowrie, I., JACKSON, N., TUdor, R., UMplebY, A., AND JONES, R. Partitioning glucose distribution/transport, disposal, and endogenous production during ivgtt. American Journal of Physiology-Endocrinology And Metabolism 282, 5 (2002), E992.

[41] HuANG, L., YI, T., AND Zou, X. On dynamics of generalized competitive and cooperative systems. Tohoku Mathematical Journal 58, 1 (2006), 89-100.

[42] JACQUEZ, J. Compartmental analysis in biology and medicine. BioMedware, 1996.

[43] JAZWINSKI, A. H. Stochastic processes and filtering theory. Courier Dover Publications, 2007.

[44] Kalman, R. E., ET AL. A new approach to linear filtering and prediction problems. Journal of basic Engineering 82, 1 (1960), 35-45.

[45] Kieffer, M., AND WAlter, E. Guaranteed nonlinear state estimator for cooperative systems. Numerical Algorithms 37, 1 (2004), 187-198.

[46] Kildegaard, J., Randløv, J., Poulsen, J. U., and Hejlesen, O. K. The impact of non-model-related variability on blood glucose prediction. Diabetes technology \& therapeutics 9, 4 (2007), 363-371. 
[47] KnOBBe, E. J., AND BucKingham, B. The extended kalman filter for continuous glucose monitoring. Diabetes technology $\mathcal{E}$ therapeutics 7,1 (2005), 15-27.

[48] KNUTH, D. E. Literate programming. The Computer Journal 27 (May 1984), 97-111.

[49] KRAEGEN, E., AND CHISHOLM, D. Insulin responses to varying profiles of subcutaneous insulin infusion: kinetic modelling studies. Diabetologia 26, 3 (1984), 208-213.

[50] KUHN, W. Rigorously computed orbits of dynamical systems without the wrapping effect. Computing 61, 1 (1998), 47-67.

[51] KunZE, H., AND SIEGEL, D. Monotonicity with respect to closed convex cones i. Dynamics of Continuous Discrete and Impulsive Systems 5 (1999), 433-450.

[52] KunZE, H., AND SIEGEL, D. Monotonicity with respect to closed convex cones ii. Applicable Analysis 77, 3-4 (2001), 233-248.

[53] KUNZE, H., AND SIEGEL, D. A graph theoretic approach to strong monotonicity with respect to polyhedral cones. Positivity 6, 2 (2002), 95-113.

[54] LENNERNAS, H., AND REGÅRDH, C. Evidence for an interaction between the $\beta$-blocker pafenolol and bile salts in the intestinal lumen of the rat leading to dose-dependent oral absorption and double peaks in the plasma concentration-time profile. Pharmaceutical research 10, 6 (1993), 879-883.

[55] LEVANT, A. Universal single-input-single-output (siso) sliding-mode controllers with finite-time convergence. Automatic Control, IEEE Transactions on 46,9 (2001), 1447-1451.

[56] LOHNER, R. Enclosing the solutions of ordinary initial and boundary value problems. Computer Arithmetic: Scientific Computation and Programming Languages 255 (1987), 286.

[57] LOHNER, R. Einschließung der Lösung gewöhnlicher Anfangs-und Randwertaufgaben und Anwendungen. PhD thesis, Universität Karlsruhe, 1988.

[58] MAKinO, K., AND BERZ, M. Cosy infinity version 8. Nuclear Instruments and Methods in Physics Research Section A: Accelerators, Spectrometers, Detectors and Associated Equipment 427, 1 (1999), 338-343.

[59] MAYBECK, P. S. Stochastic models, estimation, and control. Mathematics in Science and Engineering 141, 1 (1979), 423. 
[60] Moore, R. Interval analysis, vol. 60. Prentice-Hall Englewood Cliffs, 1966.

[61] NeDiALKOV, N. Interval tools for ODEs and DAEs. In SCAN 2006: 12th GAMM-IMACS International Symposium on Scientific Computing, Computer Arithmetic and Validated Numerics (2006), IEEE, pp. 4-4.

[62] NeDiALKOV, N. VNODE-LP: A validated solver for initial value problems in ordinary differential equations. Technical Report CAS-06-06-NN, Department of Computing and Software, McMaster University, Hamilton, Ontario, Canada, L8S 4K1, 2006.

[63] NedialKov, N., JACKSON, K., AND CORLISS, G. Validated solutions of initial value problems for ordinary differential equations. Applied Mathematics and Computation 105, 1 (1999), 21-68.

[64] Nedialkov, N. S., AND JACKSON, K. R. An interval hermiteobreschkoff method for computing rigorous bounds on the solution of an initial value problem for an ordinary differential equation. Reliable Computing 5, 3 (1999), 289-310.

[65] Neumaier, A. The wrapping effect, ellipsoid arithmetic, stability and confidence regions. Computing. Supplementum 9 (1993), 175-190.

[66] ObERlE, R., AND AMIDON, G. The influence of variable gastric emptying and intestinal transit rates on the plasma level curve of cimetidine; an explanation for the double peak phenomenon. Journal of Pharmacokinetics and Pharmacodynamics 15, 5 (1987), 529-544.

[67] Patek, S., Magni, L., Dassau, E., Karvetski, C., Toffanin, C., De Nicolao, G., Del Favero, S., Breton, M., Dalla Man, C., ReNARD, E., ET AL. Modular closed-loop control of diabetes. IEEE transactions on biomedical engineering 59, 11 (2012), 2986-2999.

[68] Pedersen, P., And Miller, R. Pharmacokinetics and bioavailability of cimetidine in humans. Journal of pharmaceutical sciences 69, 4 (1980), 394-398.

[69] Prud'homme, T., Bock, A., Francois, G., And Gillet, D. Preclinically assessed optimal control of postprandial glucose excursions for type 1 patients with diabetes. In Automation Science and Engineering (CASE), 2011 IEEE Conference on (2011), IEEE, pp. 702-707.

[70] Puckett, W., AND Lightfoot, E. A model for multiple subcutaneous insulin injections developed from individual diabetic patient data. American Journal of Physiology-Endocrinology And Metabolism 269, 6 (1995), E1115-E1124. 
[71] Puig, V., Stancu, A., And Quevedo, J. Simulation of uncertain dynamic systems described by interval models: A survey. In 16th IFAC World Congress (2005).

[72] RADZIUK, J., AND LICKLEY, H. The metabolic clearance of glucose: measurement and meaning. Diabetologia 28, 6 (1985), 315-322.

[73] Revert, A., Calm, R., Vehi, J., And Bondia, J. Calculation of the best basal-bolus combination for postprandial glucose control in insulin pump therapy. IEEE Transactions on Biomedical Engineering 58, 2 (2011), 274-281.

[74] Rossetti, P., Ampudia-Blasco, F. J., Laguna, A., Revert, A., Vehì, J., ASCASO, J. F., AND BONDIA, J. Evaluation of a novel continuous glucose monitoring-based method for mealtime insulin dosing-the ibolusin subjects with type 1 diabetes using continuous subcutaneous insulin infusion therapy: A randomized controlled trial. Diabetes Technology $\mathcal{E}$ Therapeutics 14, 11 (2012), 1043-1052.

[75] Rossetti, P., Bondia, J., Vehí, J., And FAnelli, C. G. Estimating plasma glucose from interstitial glucose: the issue of calibration algorithms in commercial continuous glucose monitoring devices. Sensors 10, 12 (2010), 10936-10952.

[76] RuMP, S. INTLAB - INTerval LABoratory. In Developments in Reliable Computing, T. Csendes, Ed. Kluwer Academic Publishers, Dordrecht, 1999, pp. 77-104.

[77] SAinz, M. Á., ARMEngol, J., AND Vehí, J. Fault detection and isolation of the three-tank system using the modal interval analysis. Journal of process control 12, 2 (2002), 325-338.

[78] Shimoda, S., Nishida, K., SAKakida, M., Konno, Y., Ichinose, K., Uehara, M., NOWAK, T., AND SHICHIRI, M. Closed-loop subcutaneous insulin infusion algorithm with a short-acting insulin analog for long-term clinical application of a wearable artificial endocrine pancreas. Frontiers of medical and biological engineering: the international journal of the Japan Society of Medical Electronics and Biological Engineering 8, 3 (1996), 197-211.

[79] Smith, H. Monotone dynamical systems: An introduction to the theory of competitive and cooperative systems. AMS Bookstore, 2008.

[80] Smith, H., AND Waltman, P. The theory of the chemostat: dynamics of microbial competition, vol. 13. Cambridge Univ Pr, 1995.

[81] Sontag, E. Monotone and near-monotone biochemical networks. Systems and Synthetic Biology 1, 2 (2007), 59-87. 
[82] STÅHL, F., AND JOHANSSON, R. Diabetes mellitus modeling and shortterm prediction based on blood glucose measurements. Mathematical biosciences 217, 2 (2009), 101-117.

[83] Swan, K. L., Dziura, J. D., Steil, G. M., Voskanyan, G. R., Sikes, K. A., Steffen, A. T., Martin, M. L., Tamborlane, W. V., And WEINZIMER, S. A. Effect of age of infusion site and type of rapid-acting analog on pharmacodynamic parameters of insulin boluses in youth with type 1 diabetes receiving insulin pump therapy. Diabetes Care 32, 2 (2009), 240-244.

[84] VORA, J., AND HEISE, T. Variability of glucose-lowering effect as a limiting factor in optimizing basal insulin therapy: a review. Diabetes, Obesity and Metabolism 15, 8 (2013), 701-712.

[85] WALCHER, S. On cooperative systems with respect to arbitrary orderings. Journal of Mathematical Analysis and Applications 263, 2 (2001), 543554.

[86] WALter, E., AND PRONZATO, L. Identification of parametric models from experimental data. Communications and Control Engineering Series. Springer, London, 1997.

[87] Whiting, D. R., Guariguata, L., Weil, C., AND ShaW, J. Idf diabetes atlas: global estimates of the prevalence of diabetes for 2011 and 2030. Diabetes research and clinical practice 94, 3 (2011), 311-321.

[88] Wilinska, M., Chassin, L., Schaller, H., Schaupp, L., Pieber, T., AND HOVORKA, R. Insulin kinetics in type-1 diabetes: continuous and bolus delivery of rapid acting insulin. IEEE Transactions on Biomedical Engineering 52, 1 (2005), 3-12.

[89] Wilinska, M. E., BodenlenZ, M., Chassin, L. J., Schaller, H. C., Schaupp, L. A., Pieber, T. R., AND HovorKA, R. Interstitial glucose kinetics in subjects with type 1 diabetes under physiologic conditions. Metabolism 53, 11 (2004), 1484-1491.

[90] Williams, G., AND PICKUP, J. C. Handbook of diabetes. Blackwell Science Oxford, 1999. 


\section{Author}

Diego de Pereda (Madrid, Spain, 29 April 1985) received his B.Sc. degree (2008) in Mathematics from the Universidad Autónoma de Madrid (Spain), carrying out a 1-year stay at the University of Nottingham (England). In 2010, he received his M.Sc. degree in Mathematical Research from the Universidad Complutense de Madrid (Spain).

Until the submission of this thesis (2015), he had been a PhD Student at the Universitat Politècnica de València (Spain), as a member of the Control of Complex Systems group and the ClosedLoop4Meal project. During his PhD, he carried out a 3-month research stay at INRIA Sophia Antipolis (France).

His main research interests are applied mathematics for biological systems, dynamical systems under parametric uncertainty, interval analysis, and the development of an artificial pancreas for the treatment of the diabetes disease. 